\title{
CARLEMAN ESTIMATES FOR THE NON-STATIONARY LAMÉ SYSTEM AND THE APPLICATION TO AN INVERSE PROBLEM
}

\author{
Oleg Yu. Imanuvilov ${ }^{1}$ and Masahiro Yamamoto $^{2}$
}

\begin{abstract}
In this paper, we establish Carleman estimates for the two dimensional isotropic nonstationary Lamé system with the zero Dirichlet boundary conditions. Using this estimate, we prove the uniqueness and the stability in determining spatially varying density and two Lamé coefficients by a single measurement of solution over $(0, T) \times \omega$, where $T>0$ is a sufficiently large time interval and a subdomain $\omega$ satisfies a non-trapping condition.
\end{abstract}

Mathematics Subject Classification. 35B60, 35R25, 35R30, 73C02.

Received February 17, 2003. Revised August 22, 2003.

\section{INTRODUCTION}

This paper is concerned with Carleman estimates for the two dimensional non-stationary isotropic Lamé system with the zero Dirichlet boundary condition and an application to an inverse problem of determining spatially varying density and the Lamé coefficients by a single interior measurement of the solution. The Carleman estimate is a weighted $L^{2}$-estimate of the solution to a partial differential equation and it has been fundamental for proving the uniqueness in a Cauchy problem for the partial differential equation or the unique continuation.

More precisely, we consider the two dimensional isotropic non-stationary Lamé system:

$$
\begin{aligned}
(P \mathbf{u})\left(x_{0}, x^{\prime}\right) & \equiv \rho\left(x^{\prime}\right) \partial_{x_{0}}^{2} \mathbf{u}\left(x_{0}, x^{\prime}\right)-\left(L_{\lambda, \mu} \mathbf{u}\right)\left(x_{0}, x^{\prime}\right)=\mathbf{f}\left(x_{0}, x^{\prime}\right), \\
x & \equiv\left(x_{0}, x^{\prime}\right) \in Q \equiv(0, T) \times \Omega,
\end{aligned}
$$

where

$$
\begin{aligned}
\left(L_{\lambda, \mu} \mathbf{v}\right)\left(x^{\prime}\right) \equiv & \mu\left(x^{\prime}\right) \Delta \mathbf{v}\left(x^{\prime}\right)+\left(\mu\left(x^{\prime}\right)+\lambda\left(x^{\prime}\right)\right) \nabla_{x^{\prime}} \operatorname{div} \mathbf{v}\left(x^{\prime}\right) \\
& +\left(\operatorname{div} \mathbf{v}\left(x^{\prime}\right)\right) \nabla_{x^{\prime}} \lambda\left(x^{\prime}\right)+\left(\nabla_{x^{\prime}} \mathbf{v}+\left(\nabla_{x^{\prime}} \mathbf{v}\right)^{T}\right) \nabla_{x^{\prime}} \mu\left(x^{\prime}\right), \quad x^{\prime} \in \Omega .
\end{aligned}
$$

Throughout this paper, $\Omega \subset \mathbb{R}^{2}$ is a bounded domain whose boundary $\partial \Omega$ is of class $C^{3}, x_{0}$ and $x^{\prime}=\left(x_{1}, x_{2}\right)$ denote the time variable and the spatial variable respectively, and $\mathbf{u}=\left(u_{1}, u_{2}\right)^{T}$ where ${ }^{T}$ denotes the transpose

Keywords and phrases. Carleman estimate, Lamé system, inverse problem.

1 Department of Mathematics, Iowa State University, 400 Carver Hall Ames IA 50011-2064 USA; e-mail: vika@iastate.edu

2 Department of Mathematical Sciences, The University of Tokyo, Komaba Meguro Tokyo 153-8914 Japan;

e-mail: myama@ms.u-tokyo.ac.jp

(c) EDP Sciences, SMAI 2005 
of matrices, $E_{k}$ is the identity matrix of the size $k \times k$,

$$
\partial_{x_{j}} \varphi=\varphi_{x_{j}}=\frac{\partial \varphi}{\partial x_{j}}, \quad j=0,1,2
$$

We set $\nabla_{x^{\prime}} \mathbf{v}=\left(\partial_{x_{k}} v_{j}\right)_{1 \leq j, k \leq 2}$ for a vector function $\mathbf{v}=\left(v_{1}, v_{2}\right)^{T}$ and $\nabla_{x^{\prime}} \phi=\left(\partial_{x_{1}} \phi, \partial_{x_{2}} \phi\right)^{T}$ for a scalar function $\phi$. Henceforth $\nabla$ means $\nabla_{x}=\left(\partial_{x_{0}}, \partial_{x_{1}}, \partial_{x_{2}}\right)$ if we do not specify.

Moreover the coefficients $\rho, \lambda, \mu$ satisfy

$$
\rho, \lambda, \mu \in C^{2}(\bar{\Omega}), \quad \rho\left(x^{\prime}\right)>0, \mu\left(x^{\prime}\right)>0, \lambda\left(x^{\prime}\right)+\mu\left(x^{\prime}\right)>0 \quad \text { for } x^{\prime} \in \bar{\Omega} .
$$

As for more details for the Lamé system, see for example, Chapter III of Duvaut and Lions [11] or Gurtin [14].

The Carleman estimate is an essential technique not only for the unique continuation, but also for solving the exact controllability and stabilizability (e.g., Bellassoued [2-4], Imanuvilov [17], Imanuvilov and Yamamoto [25], Kazemi and Klibanov [32], Tataru [44], Zhang [51], and Lasiecka and Triggiani [37] as a related book) and the inverse problems (e.g., Bukhgeim [6], Bukhgeim and Klibanov [8], Klibanov [35]). Thus the first main purpose of this paper is to establish Carleman estimates for system (1.1). Our method works, in principle, also for the three dimensional case but the arguments are more complicated and independent consideration is required. Thus in this paper, we will exclusively discuss the spatially two dimensional case. In a forthcoming paper, we will treat the three dimensional case.

Since the pioneering work [9] by Carleman, the theory of inequalities of Carleman's type has been rapidly developed and now many general results are available for a single partial differential equation (see $[12,15,29$, $30,44]$ ), while for strongly coupled systems of partial differential equations, the situation is more complicated and much less understood. To our best knowledge, the most general result for systems of partial differential equations is Calderon's uniqueness theorem (see e.g., $[12,52]$ ). The technique developed by Calderon, reduces the system of partial differential equations to a system of pseudo-differential operators of the first order:

$$
\frac{\mathrm{d} \mathbf{U}}{\mathrm{d} x_{2}}=\mathbf{M}\left(x, D_{x_{0}}, D_{x_{1}}\right) \mathbf{U}+\mathbf{F},
$$

where $\mathbf{M}\left(x, D_{x_{0}}, D_{x_{1}}\right)$ is a matrix pseudo-differential operator. Then by some change of variables $\mathbf{U}=$ $\mathbf{S}\left(x, D_{x_{0}}, D_{x_{1}}\right) \widetilde{\mathbf{U}}$, this matrix pseudo-differential operator $\mathbf{M}$ is reduced to $\mathbf{S}^{-1} \mathbf{M S}$ such that $\mathbf{S}^{-1} \mathbf{M S}$ consists of blocks of a small size located on the main diagonal and that in each block the principal symbols of all the operators located below the main diagonal are zero. In order to construct the matrix $\mathbf{S}$, the eigenvalues and eigenvectors of the matrix $\mathbf{M}\left(x, \xi_{0}, \xi_{1}\right)$ should be smooth functions of the variables $x$ and $\xi_{0}, \xi_{1} \in \mathbb{R}^{1}$ and each eigenvalue should not change the multiplicity. This condition is restrictive, especially in the case where we are looking for a Carleman estimate near boundary, and therefore the choice for a variable $x_{2}$ is limited. For example the non-stationary Lamé system does not satisfy this condition, in general. On the other hand, for the stationary Lamé system, this method works well and produces the unique continuation result from an arbitrary open subset (see [10]). See also Imanuvilov and Yamamoto [27] as for a Carleman estimate for the stationary Lamé system.

As long as the non-stationary Lamé system is concerned, it is known that thanks to the special structure of the system, the functions $\operatorname{div} \mathbf{u}$ and $\operatorname{rot} \mathbf{u}$ satisfy scalar wave equations (modulo lower order terms). The system of partial differential equations for the functions $\mathbf{u}, \operatorname{div} \mathbf{u}, \operatorname{rot} \mathbf{u}$, is coupled via only first order terms. This allows us to apply the Carleman estimate for a scalar hyperbolic equation in the case where the function $\mathbf{u}$ has a compact support (see $[13,16,19])$.

The structure of our proof is in principle similar to Yamamoto [49]. That is, we work mainly with two hyperbolic equations depending on a parameter $s>0$ for the functions $z_{\lambda+2 \mu} \equiv \mathrm{e}^{s \phi} \operatorname{div} \mathbf{u}$ and $z_{\mu} \equiv \mathrm{e}^{\mathrm{s} \phi} \operatorname{rot} \mathbf{u}$ : $P_{\lambda+2 \mu}(x, D, s) z_{\lambda+2 \mu}=(\operatorname{div} \mathbf{f}) e^{s \phi}$ and $P_{\mu}(x, D, s) z_{\mu}=(\operatorname{rot} \mathbf{f}) \mathrm{e}^{s \phi}$. The main difficulty one should overcome, is that there are no boundary conditions for these functions. This problem is solved in the following way: outside 
an exceptional set in the contangent bundle $T^{*}(Q)$, the operators $P_{\lambda+2 \mu}$ and $P_{\mu}$ can be microlocally factorized as the product of some function $\widetilde{\beta}(x)$ and two pseudo-differential operators of the first order:

$$
P_{\beta}(x, D, s)=\widetilde{\beta} P_{-, \beta}(x, D, s) P_{+, \beta}(x, D, s),
$$

where $\beta=\lambda+2 \mu$ or $=\mu, P_{ \pm, \beta}=D_{x_{2}}-\Gamma_{\beta}^{ \pm}\left(x, D_{x_{0}}, D_{x_{1}}, s\right)$, and $x_{2}$ is normal to the boundary $\partial \Omega$. Since the principal symbol of the operator $\Gamma_{\beta}^{-}\left(x, \xi_{0}, \xi_{1}, s\right)$ satisfies the inequality

$$
-\operatorname{Im} \Gamma_{\beta}^{-}\left(x, \xi_{0}, \xi_{1}, s\right) \geq C|s|
$$

with a constant $C>0$, we have a priori estimates for $\left.P_{+, \beta}(x, D, s) z_{\beta}\right|_{x_{2}=0}$ in an $L^{2}$-space. These estimates and the zero Dirichlet boundary condition yield the $H^{1}$-boundary estimates for $z_{\beta}$. The set on which we cannot factorize both the operators $P_{\beta}(x, D, s)$ into a product of the first order operators, has to be discussed separately.

Next we will prove a Carleman estimate with the $H^{-1}(Q)$ norm of the force $\mathbf{f}$ in the right hand side. The Carleman estimate with right hand side in $H^{-1}(Q)$-space was proved by Imanuvilov [18], Ruiz [43], for a scalar hyperbolic equation and by Imanuvilov and Yamamoto [26] for a parabolic equation. In this paper, by a method in [26], we will derive an $H^{-1}(Q)$-Carleman estimate (Th. 2.3) for (1.1) from a Carleman estimate (Th. 2.1) with $H^{1}$-norm.

Finally we consider an inverse problem of determining the coefficients $\lambda, \mu$ and $\rho$ from one single measurement of the solution $\mathbf{u}$ in $(0, T) \times \omega$, where $\omega \subset \Omega$ is a suitable subdomain and $T>0$ is sufficiently large. By our $H^{-1}(Q)$-Carleman estimate for the Lamé system, we will establish the uniqueness and the stability result for the inverse problem.

This paper is composed of nine sections and two appendices. In Section 2, we state Carleman estimates (Ths. 2.1-2.3) for functions which do not have compact supports but satisfy the zero Dirichlet boundary condition on $(0, T) \times \partial \Omega$. Theorem 2.1 is a Carleman estimate whose right hand side is estimated in $H^{1}$-space. Theorems 2.2 and 2.3 are Carleman estimates respectively with right hand sides in $L^{2}$-space and in $H^{-1}$-space. In Section 3, we will apply the $H^{-1}$-Carleman estimate (Th. 2.3), and prove the uniqueness and the conditional stability in the inverse problem with a single interior measurement. In Sections 4-8, we prove Theorem 2.1; In Section 4, we will reduce Theorem 2.1 to Lemma 4.1, and in Section 5, we further localize Lemma 4.1 by means of pseudo-differential operators. Dividing all the possible cases into three cases, in Sections 6-8, we will complete the proof of the localized estimate separately in those three cases. Finally Theorems 2.2 and 2.3 are proved in Section 9.

\section{Carleman estimates for the two dimensional non-Stationary Lamé system}

Let us consider the two dimensional Lamé system

$$
\begin{gathered}
P \mathbf{u}\left(x_{0}, x^{\prime}\right) \equiv \rho\left(x^{\prime}\right) \partial_{x_{0}}^{2} \mathbf{u}\left(x_{0}, x^{\prime}\right)-\left(L_{\lambda, \mu} \mathbf{u}\right)\left(x_{0}, x^{\prime}\right)=\mathbf{f}\left(x_{0}, x^{\prime}\right) \quad \text { in } Q \\
\left.\mathbf{u}\right|_{(0, T) \times \partial \Omega}=0, \quad \mathbf{u}\left(T, x^{\prime}\right)=\partial_{x_{0}} \mathbf{u}\left(T, x^{\prime}\right)=\mathbf{u}\left(0, x^{\prime}\right)=\partial_{x_{0}} \mathbf{u}\left(0, x^{\prime}\right)=0
\end{gathered}
$$

where $\mathbf{u}=\left(u_{1}, u_{2}\right)^{T}, \mathbf{f}=\left(f_{1}, f_{2}\right)^{T}$ are vector-valued functions, and the partial differential operator $L_{\lambda, \mu}$ is defined by (1.2). The coefficients $\rho, \lambda, \mu \in C^{2}(\bar{\Omega})$ are assumed to satisfy (1.3).

Let $\omega \subset \Omega$ be an arbitrarily fixed subdomain (not necessary connected). Denote by $\vec{n}\left(x^{\prime}\right)=\left(n_{1}\left(x^{\prime}\right), n_{2}\left(x^{\prime}\right)\right)$ and $\vec{\tau}\left(x^{\prime}\right)$ the outward unit normal vector and a unit tangential vector to $\partial \Omega$ at $x^{\prime}$ respectively, and set $\frac{\partial v}{\partial \vec{n}}=$ $\nabla_{x^{\prime}} v \cdot \vec{n}$ and $\frac{\partial v}{\partial \vec{\tau}}=\nabla_{x^{\prime}} v \cdot \vec{\tau}$.

We set

$$
Q_{\omega}=(0, T) \times \omega
$$


Let $\xi=\left(\xi_{0}, \xi^{\prime}\right)=\left(\xi_{0}, \xi_{1}, \xi_{2}\right)$. We set

$$
\left\{\begin{array}{l}
p_{1}(x, \xi)=\rho\left(x^{\prime}\right) \xi_{0}^{2}-\mu\left(x^{\prime}\right)\left(\left|\xi_{1}\right|^{2}+\left|\xi_{2}\right|^{2}\right) \\
p_{2}(x, \xi)=\rho\left(x^{\prime}\right) \xi_{0}^{2}-\left(\lambda\left(x^{\prime}\right)+2 \mu\left(x^{\prime}\right)\right)\left(\left|\xi_{1}\right|^{2}+\left|\xi_{2}\right|^{2}\right)
\end{array}\right.
$$

and $\nabla_{\xi}=\left(\partial_{\xi_{0}}, \partial_{\xi_{1}}, \partial_{\xi_{2}}\right)$. For arbitrary smooth functions $\varphi(x, \xi)$ and $\psi(x, \xi)$, we define the Poisson bracket by the formula

$$
\{\varphi, \psi\}=\sum_{j=0}^{2}\left(\partial_{\xi_{j}} \varphi\right)\left(\partial_{x_{j}} \psi\right)-\left(\partial_{\xi_{j}} \psi\right)\left(\partial_{x_{j}} \varphi\right) .
$$

We set $i=\sqrt{-1}$ and $\langle a, b\rangle=\sum_{k=1}^{3} a_{k} \bar{b}_{k}$ for $a=\left(a_{1}, a_{2}, a_{3}\right), b=\left(b_{1}, b_{2}, b_{3}\right) \in \mathbb{C}^{3}$.

We assume that the density $\rho$, the Lamé coefficients $\lambda, \mu$ and the domains $\Omega$, $\omega$ satisfy the following condition (cf. [15]).

Condition 2.1. There exists a function $\psi \in C^{3}(\bar{Q})$ such that $\left|\nabla_{x}^{\prime} \psi\right| \neq 0$ on $\overline{Q \backslash Q_{\omega}}$ and

$$
\begin{gathered}
\left\{p_{k},\left\{p_{k}, \psi\right\}\right\}(x, \xi)>0, \quad \forall k \in\{1,2\} \\
\text { if }(x, \xi) \in\left(\overline{Q \backslash Q_{\omega}}\right) \times\left(\mathbb{R}^{3} \backslash\{0\}\right) \text { satisfies } p_{k}(x, \xi)=\left\{p_{k}, \psi\right\}(x, \xi)=0, \\
\left\{p_{k}(x, \xi-i s \nabla \psi(x)), p_{k}(x, \xi+i s \nabla \psi(x))\right\} / 2 i s>0, \quad \forall k \in\{1,2\} \\
\text { if }(x, \xi, s) \in\left(\overline{Q \backslash Q_{\omega}}\right) \times\left(\mathbb{R}^{3} \backslash\{0\}\right) \times(\mathbb{R} \backslash\{0\}) \text { satisfies } \\
p_{k}(x, \xi+i s \nabla \psi(x))=\left\langle\nabla_{\xi} p_{k}(x, \xi+i s \nabla \psi(x)), \nabla \psi(x)\right\rangle=0 .
\end{gathered}
$$

On the lateral boundary, we assume

$$
\begin{aligned}
\sqrt{\rho}\left|\psi_{x_{0}}\right|<\frac{\mu}{\sqrt{\lambda+2 \mu}}\left|\frac{\partial \psi}{\partial \vec{\tau}}\right|+\frac{\sqrt{\mu} \sqrt{\lambda+\mu}}{\sqrt{\lambda+2 \mu}}\left|\frac{\partial \psi}{\partial \vec{n}}\right|, \quad p_{1}(x, \nabla \psi)<0, \quad \forall x \in \overline{(0, T) \times \partial \Omega} \\
\quad \text { and } \frac{\partial \psi}{\partial \vec{n}} \mid \frac{}{(0, T) \times(\partial \Omega \backslash \partial \omega)}<0 .
\end{aligned}
$$

Let $\psi(x)$ be the weight function in Condition 2.1. Using this function, we introduce the function $\phi(x)$ by

$$
\phi(x)=\mathrm{e}^{\tau \psi(x)}, \quad \tau>1,
$$

where the parameter $\tau>0$ will be fixed below. Denote

$$
\begin{aligned}
\|\mathbf{u}\|_{\mathcal{B}(\phi, Q)}^{2} \equiv & \int_{Q}\left(\sum_{|\alpha|=0}^{2} s^{4-2|\alpha|}\left|\partial_{x}^{\alpha} \mathbf{u}\right|^{2}+s|\nabla \operatorname{rot} \mathbf{u}|^{2}+s^{3}|\operatorname{rot} \mathbf{u}|^{2}\right. \\
& \left.+s|\nabla \operatorname{div} \mathbf{u}|^{2}+s^{3}|\operatorname{div} \mathbf{u}|^{2}\right) \mathrm{e}^{2 s \phi} \mathrm{d} x
\end{aligned}
$$

where $\alpha=\left(\alpha_{0}, \alpha_{1}, \alpha_{2}\right), \alpha_{j} \in \mathbb{N}_{+} \cup\{0\}, \partial_{x}^{\alpha}=\partial_{x_{0}}^{\alpha_{0}} \partial_{x_{1}}^{\alpha_{1}} \partial_{x_{2}}^{\alpha_{2}}$.

Now we formulate our Carleman estimates as main results. 
Theorem 2.1. Let $\mathbf{f} \in\left(H^{1}(Q)\right)^{2}$, and let the function $\psi$ satisfy Condition 2.1. Then there exists $\widehat{\tau}>0$ such that for any $\tau>\widehat{\tau}$, there exists $s_{0}=s_{0}(\tau)>0$ such that for any solution $\mathbf{u} \in\left(H^{1}(Q)\right)^{2} \cap L^{2}\left(0, T ;\left(H^{2}(\Omega)^{2}\right)\right.$ to problem (2.1)-(2.2), the following estimate holds true:

$$
\begin{aligned}
\|\mathbf{u}\|_{Y(\phi, Q)}^{2} \triangleq\|\mathbf{u}\|_{\mathcal{B}(\phi, Q)}^{2}+s\left\|\frac{\partial \mathbf{u}}{\partial \vec{n}} \mathrm{e}^{s \phi}\right\|_{\left(H^{1}((0, T) \times \partial \Omega)\right)^{2}}^{2}+s\left\|\frac{\partial^{2} \mathbf{u}}{\partial \vec{n}^{2}} \mathrm{e}^{s \phi}\right\|_{\left(L^{2}((0, T) \times \partial \Omega)\right)^{2}}^{2}+s^{3}\left\|\frac{\partial \mathbf{u}}{\partial \vec{n}} \mathrm{e}^{s \phi}\right\|_{\left(L^{2}((0, T) \times \partial \Omega)\right)^{2}}^{2} \\
\leq C_{1}\left(s^{2}\left\|\mathrm{fe}^{s \phi}\right\|_{\left(L^{2}(Q)\right)^{2}}^{2}+\left\|(\nabla \mathbf{f}) \mathrm{e}^{s \phi}\right\|_{\left(L^{2}(Q)\right)^{2}}^{2}+\|\mathbf{u}\|_{\mathcal{B}\left(\phi, Q_{\omega}\right)}^{2}\right), \quad \forall s \geq s_{0}(\tau), \quad(2.9
\end{aligned}
$$

where the constant $C_{1}=C_{1}(\tau)>0$ is independent of $s$.

Remark. In Carleman estimate (2.9), the weights which correspond to rot $\mathbf{u}$ and div $\mathbf{u}$ are better than the weights which correspond to $\nabla \mathbf{u}$. This is a result of the special structure of the Lamé system which allows us to decouple into two wave equations for $\operatorname{rot} \mathbf{u}$ and $\operatorname{div} \mathbf{u}$ (see (4.1)).

Next we formulate other two Carleman estimates where norms of the function $\mathbf{f}$ are taken in $\left(L^{2}(Q)\right)^{2}$ and $H^{-1}(Q)$. In particular, the second of these two Carleman estimate is essential for obtaining our sharp uniqueness result in the inverse problem.

In addition to Condition 2.1, we assume

$$
\partial_{x_{0}} \psi\left(T, x^{\prime}\right)<0, \quad \partial_{x_{0}} \psi\left(0, x^{\prime}\right)>0, \quad \forall x^{\prime} \in \bar{\Omega}
$$

Theorem 2.2. Let $\mathbf{f} \in\left(L^{2}(Q)\right)^{2}$ and let the function $\psi$ satisfy (2.10) and Condition 2.1 and let function $\phi$ be given by (2.7). Then there exists $\widehat{\tau}>0$ such that for any $\tau>\widehat{\tau}$, there exists $s_{0}=s_{0}(\tau)>0$ such that for any solution $\mathbf{u} \in\left(H^{1}(Q)\right)^{2}$ to problem (2.1)-(2.2), the following estimate holds true:

$$
\int_{Q}\left(|\nabla \mathbf{u}|^{2}+s^{2}|\mathbf{u}|^{2}\right) \mathrm{e}^{2 s \phi} \mathrm{d} x \leq C_{1}\left(\left\|\mathbf{f} \mathrm{e}^{s \phi}\right\|_{\left(L^{2}(Q)\right)^{2}}^{2}+\int_{Q_{\omega}}\left(|\nabla \mathbf{u}|^{2}+s^{2}|\mathbf{u}|^{2}\right) \mathrm{e}^{2 s \phi} \mathrm{d} x\right), \quad \forall s \geq s_{0}(\tau),
$$

where the constant $C_{1}=C_{1}(\tau)>0$ is independent of $s$.

Theorem 2.3. Let $\mathbf{f}=\mathbf{f}_{-1}+\sum_{j=0}^{2} \partial_{x_{j}} \mathbf{f}_{j}$ with $\mathbf{f}_{-1} \in\left(H^{-1}(Q)\right)^{2}$ and $\mathbf{f}_{0}, \mathbf{f}_{1}, \mathbf{f}_{2} \in\left(L^{2}(Q)\right)^{2}$, and let the function $\psi$ satisfy (2.10) and Condition 2.1 and let the function $\phi$ be given by (2.7). Then there exists $\widehat{\tau}>0$ such that for any $\tau>\widehat{\tau}$, there exists $s_{0}=s_{0}(\tau)>0$ such that for any solution $\mathbf{u} \in\left(L^{2}(Q)\right)^{2}$ to problem (2.1)-(2.2), the following estimate holds true:

$$
\int_{Q}|\mathbf{u}|^{2} \mathrm{e}^{2 s \phi} \mathrm{d} x \leq C_{1}\left(\left\|\mathbf{f}_{-1} \mathrm{e}^{s \phi}\right\|_{\left(H^{-1}(Q)\right)^{2}}^{2}+\sum_{j=0}^{2}\left\|\mathbf{f}_{j} \mathrm{e}^{s \phi}\right\|_{\left(L^{2}(Q)\right)^{2}}^{2}+\int_{Q_{\omega}}|\mathbf{u}|^{2} \mathrm{e}^{2 s \phi} \mathrm{d} x\right), \quad \forall s \quad \geq \quad s_{0}(\tau),
$$

where the constant $C_{1}=C_{1}(\tau)>0$ is independent of $s$.

\section{Determination of the Density AND the LAmÉ COEFFicients By A SingLE MEASUREMENT}

Recall that the differential operator $L_{\lambda, \mu}$ is defined by (1.2). We assume (1.3) for $\rho, \lambda, \mu$. By $\mathbf{u}=$ $\mathbf{u}(\lambda, \mu, \rho, \mathbf{p}, \mathbf{q}, \eta)(x)$, we denote the sufficiently smooth solution to 


$$
\begin{gathered}
\rho\left(x^{\prime}\right)\left(\partial_{x_{0}}^{2} \mathbf{u}\right)(x)=\left(L_{\lambda, \mu} \mathbf{u}\right)(x), \quad x \in Q, \\
\mathbf{u}(x)=\eta(x), \quad x \in(0, T) \times \partial \Omega, \\
\mathbf{u}\left(T / 2, x^{\prime}\right)=\mathbf{p}\left(x^{\prime}\right), \quad\left(\partial_{x_{0}} \mathbf{u}\right)\left(T / 2, x^{\prime}\right)=\mathbf{q}\left(x^{\prime}\right), \quad x^{\prime} \in \Omega,
\end{gathered}
$$

where $\eta, \mathbf{p}$ and $\mathbf{q}$ are suitably given functions.

Let $\omega \subset \Omega$ be a suitably given subdomain. We consider

Inverse Problem. Let $\mathbf{p}_{j}, \mathbf{q}_{j}, \eta_{j}, 1 \leq j \leq \mathcal{N}$, be appropriately given. Then determine $\lambda\left(x^{\prime}\right), \mu\left(x^{\prime}\right), \rho\left(x^{\prime}\right)$, $x^{\prime} \in \Omega$, by

$$
\mathbf{u}\left(\lambda, \mu, \rho, \mathbf{p}_{j}, \mathbf{q}_{j}, \eta_{j}\right)(x), \quad x \in Q_{\omega} \equiv(0, T) \times \omega .
$$

Our formulation of the inverse problem is one with a finite number of observations (i.e., $\mathcal{N}<\infty$ ). For inverse problems for the non-stationary Lamé equation by infinitely many boundary observations (i.e., Dirichlet-toNeumann map), we refer to Rachele [42], for example. A monograph of Yahkno [48] is concerned with the inverse problems for the Lamé system.

For the formulation with a finite number of observations, Bukhgeim and Klibanov [8] proposed a remarkable method based on a Carleman estimate and established the uniqueness for similar inverse problems for scalar partial differential equations. As works after [8], see:

(1) Baudouin and Puel [5], Bukhgeim [6] for an inverse problem of determining potentials in Schrödinger equations;

(2) Imanuvilov and Yamamoto [21], Isakov [29,30], Klibanov [35] for the corresponding inverse problems for parabolic equations;

(3) Bukhgeim, Cheng, Isakov and Yamamoto [7], Imanuvilov and Yamamoto [22-24], Isakov [28-30], Isakov and Yamamoto [31], Khădarov [33,34], Klibanov [35], Puel and Yamamoto [40,41], Yamamoto [50] for inverse problems of determining potentials, damping coefficients or the principal terms in scalar hyperbolic equations.

In particular, for inverse hyperbolic equations, we have to assume that the observation subdomain $\omega$ should satisfy a geometric condition and the observation time $T$ has to be sufficiently large, which is a natural consequence of the hyperbolicity of the governing partial differential equations. Such situations are similar for our inverse problem for the Lamé system.

The Carleman estimate for the non-stationary Lamé equation was obtained for functions with compact supports, by Eller, Isakov, Nakamura and Tataru [13], Ikehata, Nakamura and Yamamoto [16], Imanuvilov, Isakov and Yamamoto [19], Isakov [28], and, by the methodology by [8] or [22], several uniqueness results are available for the inverse problem for Lamé system (3.1)-(3.3): [28] established the uniqueness in determining a single coefficient $\rho\left(x^{\prime}\right)$, using four measurements $($ i.e., $\mathcal{N}=4)$.

Later [16] reduced the number of measurements to three (i.e., $\mathcal{N}=3$ ) for determining $\rho$.

Recently [19] proved conditional stability and the uniqueness in the determination of the three functions $\lambda\left(x^{\prime}\right)$, $\mu\left(x^{\prime}\right), \rho\left(x^{\prime}\right), x^{\prime} \in \Omega$, with only two measurements (i.e., $\mathcal{N}=2$ ).

In all the papers $[16,19,28]$, the authors have to assume that $\partial \omega \supset \partial \Omega$ because the basic Carleman estimates require that solutions under consideration have compact supports in $Q$.

In [28] and [16], the key is a Carleman estimate where the right hand side is estimated in an $L^{2}$-space with the divergence and the estimate is proved via a system of hyperbolic equations of $\mathbf{u}$ and div $\mathbf{u}$ with the same principal terms. On the other hand, in [19], the key is a Carleman estimate with $L^{2}$-right hand side where $\left\|\mathrm{e}^{s \phi} \operatorname{div} \mathbf{u}\right\|_{L^{2}(Q)}^{2}$ is reduced to $\left\|\mathbf{u} \mathrm{e}^{s \phi}\right\|_{L^{2}(Q)}^{2}$ by means of an $H^{-1}$-Carleman estimate for a scalar hyperbolic equation. In [19], as its consequence, we can reduce $\mathcal{N}$ to take $\mathcal{N}=2$ for simultaneous determination of all the three functions $\lambda, \mu, \rho$.

In this section, we will further apply a Carleman estimate (Th. 2.3) whose right hand side is estimated in $H^{-1}$ space to prove the conditional stability and the uniqueness with a single measurement: $\mathcal{N}=1$. Thus the main achievements are 
(1) the reduction of the number of observations, i.e., $\mathcal{N}=1$. The previous paper [19] requires $\mathcal{N}=2$;

(2) the relaxation of the assumptions on the observation subdomain $\omega$.

We will be able to prove similar results on the uniqueness and the stability in the three dimensional case on the basis of the corresponding Carleman estimate, and in a forthcoming paper, we will discuss the details.

In order to formulate our main result, we will introduce notations and an admissible set of unknown parameters $\lambda, \mu, \rho$. Henceforth we set $\left(x^{\prime}, y^{\prime}\right)=\sum_{j=1}^{2} x_{j} y_{j}$ for $x^{\prime}=\left(x_{1}, x_{2}\right)$ and $y^{\prime}=\left(y_{1}, y_{2}\right)$. Let a subdomain $\omega \subset \Omega$ satisfy

$$
\partial \omega \supset\left\{x^{\prime} \in \partial \Omega ;\left(\left(x^{\prime}-y^{\prime}\right), \vec{n}\left(x^{\prime}\right)\right) \geq 0\right\} \equiv \Gamma
$$

with some $y^{\prime} \notin \bar{\Omega}$.

Remark. Under Condition (3.5) on $\omega$, we can prove the observability inequality for the wave equation $\partial_{x_{0}}^{2}-\Delta$ if the observation time $T$ is larger than $2 \sup _{x^{\prime} \in \Omega}\left|x^{\prime}-y^{\prime}\right|$ (e.g., [39]). If (3.5) holds and $T>0$ is sufficiently large, then $\omega$ and $T$ satisfy the geometric optics condition in [1], so that we can prove observability inequalites. On the other hand, for solving inverse problems, a Carleman estimate is essential and observability inequalities are not directly applicable. If for other $\omega$ and $T>0$, we will be able to verify Condition 2.1 similarly to Lemma 3.1 or [24], then we can establish similar results to Theorem 3.1 below. However searches for other $\omega$ and $T$ are omitted here because those are lengthy.

Denote

$$
d=\left(\sup _{x^{\prime} \in \Omega}\left|x^{\prime}-y^{\prime}\right|^{2}-\inf _{x^{\prime} \in \Omega}\left|x^{\prime}-y^{\prime}\right|^{2}\right)^{\frac{1}{2}} .
$$

Next we define an admissible set of unknown coefficients $\lambda, \mu, \rho$. Let $M_{0} \geq 0,0<\theta_{0} \leq 1$ and $\theta_{1}>0$ be arbitrarily fixed and let us introduce the conditions on a function $\beta$ :

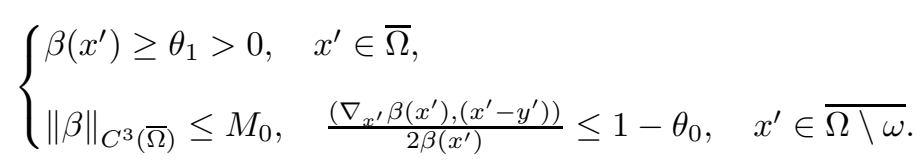

For fixed functions $a, b, \eta$ on $\partial \Omega$ and $\mathbf{p}, \mathbf{q}$ in $\Omega$, we set

$$
\begin{aligned}
& \mathcal{W}=\mathcal{W}_{M_{0}, M_{1}, \theta_{0}, \theta_{1}, a, b}=\left\{(\lambda, \mu, \rho) \in\left(C^{3}(\bar{\Omega})\right)^{3} ; \lambda=a, \mu=b \quad \text { on } \partial \Omega\right. \\
& \left.\frac{\lambda+2 \mu}{\rho}, \frac{\mu}{\rho} \text { satisfy }(3.7), \frac{\min \left\{\mu^{2}\left(x^{\prime}\right), \mu\left(x^{\prime}\right)(\lambda+\mu)\left(x^{\prime}\right)\right\}}{\rho\left(x^{\prime}\right)(\lambda+2 \mu)\left(x^{\prime}\right)} \geq \theta_{1}>0, x^{\prime} \in \bar{\Omega},\|\mathbf{u}(\lambda, \mu, \rho, \mathbf{p}, \mathbf{q}, \eta)\|_{W^{7, \infty}(Q)} \leq M_{1}\right\}
\end{aligned}
$$

where the constant $M_{1}$ is given.

Remark. The admissible set $\mathcal{W}$ is restrictive, but contains sufficiently many $(\lambda, \mu, \rho)$. We here give a subset of $\mathcal{W}$ which suggests that the set $\mathcal{W}$ is not very small. Let $\mathbf{p}, \mathbf{q} \in C^{\infty}(\bar{\Omega})$ be given arbitrarily and let us choose arbitrary positive constants $a, b, \rho_{0}$. Then, for the Dirichlet boundary data $\eta \in C^{\infty}([0, T] \times \partial \Omega)$, we assume

$$
\begin{aligned}
& \left(\partial_{x_{0}}^{2 j} \eta\right)\left(T / 2, x^{\prime}\right)=\left(\frac{1}{\rho_{0}} L_{a, b}\right)^{j} \mathbf{p}\left(x^{\prime}\right), \quad\left(\partial_{x_{0}}^{2 j+1} \eta\right)\left(T / 2, x^{\prime}\right)=\left(\frac{1}{\rho_{0}} L_{a, b}\right)^{j} \mathbf{q}\left(x^{\prime}\right), \\
& x^{\prime} \in \partial \Omega, 0 \leq j \leq N_{0} .
\end{aligned}
$$

Here $N_{0}$ is a sufficiently large natural number. 
We set

$$
\begin{aligned}
& \mathcal{W}_{0}=\left\{(\lambda, \mu, \rho) \in\left(C^{\infty}(\bar{\Omega})\right)^{3} ; \lambda=a, \mu=b, \rho=\rho_{0} \quad \text { in a neighbourhood of } \partial \Omega\right. \\
& \left(\frac{\lambda+2 \mu}{\rho}\right)\left(x^{\prime}\right)>\theta_{1}, \quad\left(\frac{\mu}{\rho}\right)\left(x^{\prime}\right)>\theta_{1}, \quad \frac{\min \left\{\mu^{2}\left(x^{\prime}\right), \mu\left(x^{\prime}\right)(\lambda+\mu)\left(x^{\prime}\right)\right\}}{\rho\left(x^{\prime}\right)(\lambda+2 \mu)\left(x^{\prime}\right)}>\theta_{1}, \quad x^{\prime} \in \bar{\Omega} \\
& \|\rho\|_{C^{\infty}(\bar{\Omega})},\|\lambda\|_{C^{\infty}(\bar{\Omega})},\|\mu\|_{C^{\infty}(\bar{\Omega})}<M_{0}, \\
& \left.\left\|\frac{\rho}{2(\lambda+2 \mu)} \nabla\left(\frac{\lambda+2 \mu}{\rho}\right)\right\|_{C(\bar{\Omega})},\left\|\frac{\rho}{2 \mu} \nabla\left(\frac{\mu}{\rho}\right)\right\|_{C(\bar{\Omega})}<\frac{1-\theta_{0}}{\sup _{x^{\prime} \in \Omega \backslash \omega}\left|x^{\prime}-y^{\prime}\right|}\right\} .
\end{aligned}
$$

The set $\mathcal{W}_{0}$ is not empty and is not "thin". Then, since the conditions on $\eta$ yield compatibility conditions of sufficient orders with $\mathbf{p}, \mathbf{q}$ at $\{T / 2\} \times \partial \Omega$ for any $(\lambda, \mu, \rho) \in \mathcal{W}_{0}$, we can prove by an argument similar to [pp. 1369$1370,19]$ that $\mathbf{u}(\lambda, \mu, \rho, \mathbf{p}, \mathbf{q}, \eta) \in C^{7}(\bar{Q})$ and there exists a constant $M_{1}=M_{1}\left(a, b, \rho_{0}, \theta_{1}, M_{0}, \mathbf{p}, \mathbf{q}, \eta\right)>0$ such that

$$
\|\mathbf{u}(\lambda, \mu, \rho, \mathbf{p}, \mathbf{q}, \eta)\|_{C^{7}(\bar{Q})} \leq M_{1}
$$

for all $(\lambda, \mu, \rho) \in \mathcal{W}_{0}$. Therefore we see that $\mathcal{W}_{0}$ is a subset of $\mathcal{W}=\mathcal{W}_{M_{0}, M_{1}, \theta_{0}, \theta_{1}, a, b}$ defined by (3.8). Thus, after a suitable choice of $\eta$, we can conclude that the admissible set $\mathcal{W}$ can contain sufficiently many elements.

It is rather restrictive that $\frac{\lambda+2 \mu}{\rho}$ and $\frac{\mu}{\rho}$ should satisfy (3.7), which is one possible sufficient condition for the pseudoconvexity (i.e., Condition (2.1)). We can relax Condition (3.7) to a more generous condition which can be related with a necessary condition enabling us to establish a Carleman estimate. See Imanuvilov, Isakov and Yamamoto [20], where a scalar hyperbolic equation is discussed but the modification to the Lamé system is straightforward. Such a relaxed condition guarantees that the geodesics which are generated by the hyperbolic equations defined by (2.3), cannot remain on the level sets given by the weight function $\phi$. In particular, by $[20]$, we can replace the condition that $\frac{\lambda+2 \mu}{\rho}$ and $\frac{\mu}{\rho}$ satisfy (3.7) by one that the Hessians

$$
\left(\partial_{x_{j}} \partial_{x_{k}}\left(\frac{\rho}{\mu}\right)^{\frac{1}{2}}\right)_{1 \leq j, k \leq 2}, \quad\left(\partial_{x_{j}} \partial_{x_{k}}\left(\frac{\rho}{\lambda+2 \mu}\right)^{\frac{1}{2}}\right)_{1 \leq j, k \leq 2}
$$

are non-negative and $\left|\nabla\left(\frac{\rho}{\mu}\right)\right| \neq 0$ and $\left|\nabla\left(\frac{\rho}{\lambda+2 \mu}\right)\right| \neq 0$ on $\bar{\Omega}$.

We choose $\theta>0$ such that

$$
\theta+\frac{M_{0} d}{\sqrt{\theta_{1}}} \sqrt{\theta}<\theta_{0} \theta_{1}, \quad \theta_{1} \inf _{x^{\prime} \in \Omega}\left|x^{\prime}-y^{\prime}\right|^{2}-\theta d^{2}>0
$$

Here we note that since $y^{\prime} \notin \bar{\Omega}$, such $\theta>0$ exists.

Let $[\cdot]_{1}$ denote the first component of the vector under consideration and let $E_{2}$ the $2 \times 2$ identity matrix. We note that $\left(L_{\lambda, \mu} \mathbf{p}\right)\left(x^{\prime}\right)$, etc., are 2-column vectors for 2 -column vectors $\mathbf{p}$. Let $(\lambda, \mu, \rho)$ be an arbitrary element of $\mathcal{W}$.

Now we are ready to state

Theorem 3.1. We assume that

$$
\Omega=\left\{\left(x_{1}, x_{2}\right) ; \gamma_{0}\left(x_{2}\right)<x_{1}<\gamma_{1}\left(x_{2}\right), x_{2} \in I\right\}
$$

with some open interval $I$ and $\gamma_{0}, \gamma_{1} \in C(\bar{I})$. Moreover we assume that the functions $\mathbf{p}=\left(p_{1}, p_{2}\right)^{T}$ and $\mathbf{q}=\left(q_{1}, q_{2}\right)^{T}$ satisfy

$$
\operatorname{det}\left(\begin{array}{ccc}
\left(L_{\lambda, \mu} \mathbf{p}\right)\left(x^{\prime}\right) & \left(\operatorname{div} \mathbf{p}\left(x^{\prime}\right)\right) E_{2} & \left(\nabla_{x^{\prime}} \mathbf{p}\left(x^{\prime}\right)+\left(\nabla_{x^{\prime}} \mathbf{p}\left(x^{\prime}\right)\right)^{T}\right)\left(x^{\prime}-y^{\prime}\right) \\
\left(L_{\lambda, \mu} \mathbf{q}\right)\left(x^{\prime}\right) & \left(\operatorname{div} \mathbf{q}\left(x^{\prime}\right)\right) E_{2} & \left(\nabla_{x^{\prime}} \mathbf{q}\left(x^{\prime}\right)+\left(\nabla_{x^{\prime}} \mathbf{q}\left(x^{\prime}\right)\right)^{T}\right)\left(x^{\prime}-y^{\prime}\right)
\end{array}\right) \neq 0, \forall x^{\prime} \in \bar{\Omega}
$$




$$
\begin{aligned}
& \operatorname{det}\left(\begin{array}{lll}
\left(L_{\lambda, \mu} \mathbf{p}\right)\left(x^{\prime}\right) & \nabla_{x^{\prime}} \mathbf{p}\left(x^{\prime}\right)+\left(\nabla_{x^{\prime}} \mathbf{p}\left(x^{\prime}\right)\right)^{T} & (\operatorname{div} \mathbf{p})\left(x^{\prime}-y^{\prime}\right) \\
\left(L_{\lambda, \mu} \mathbf{q}\right)\left(x^{\prime}\right) & \nabla_{x^{\prime}} \mathbf{q}\left(x^{\prime}\right)+\left(\nabla_{x^{\prime}} \mathbf{q}\left(x^{\prime}\right)\right)^{T} & (\operatorname{div} \mathbf{q})\left(x^{\prime}-y^{\prime}\right)
\end{array}\right) \neq 0, \forall x^{\prime} \in \bar{\Omega}, \\
& x_{1}-y_{1} \neq 0, \\
& {\left[L_{\lambda, \mu} \mathbf{q}\right]_{1}\left(\partial_{x_{1}} p_{2}+\partial_{x_{2}} p_{1}\right)\left(x^{\prime}\right) \neq\left[L_{\lambda, \mu} \mathbf{p}\right]_{1}\left(\partial_{x_{1}} q_{2}+\partial_{x_{2}} q_{1}\right)\left(x^{\prime}\right), \quad \forall x^{\prime} \in \bar{\Omega}}
\end{aligned}
$$

and that

$$
T>\frac{2}{\sqrt{\theta}} d
$$

Then there exist constants $\kappa=\kappa(\mathcal{W}, \omega, \Omega, T, \lambda, \mu, \rho) \in(0,1)$ and $C_{1}=C_{1}(\mathcal{W}, \omega, \Omega, T, \lambda, \mu, \rho)>0$ such that

$$
\begin{aligned}
& \|\widetilde{\lambda}-\lambda\|_{L^{2}(\Omega)}+\|\widetilde{\mu}-\mu\|_{L^{2}(\Omega)}+\|\widetilde{\rho}-\rho\|_{H^{-1}(\Omega)} \\
\leq & C_{1}\|\mathbf{u}(\lambda, \mu, \rho, \mathbf{p}, \mathbf{q}, \eta)-\mathbf{u}(\widetilde{\lambda}, \widetilde{\mu}, \widetilde{\rho}, \mathbf{p}, \mathbf{q}, \eta)\|_{H^{4}\left(0, T ;\left(L^{2}(\omega)\right)^{2}\right)}^{\kappa}
\end{aligned}
$$

for any $(\widetilde{\lambda}, \widetilde{\mu}, \widetilde{\rho}) \in \mathcal{W}$.

As for the corresponding results on the stability for inverse problems for scalar hyperbolic equations, we refer to $[22-24]$ for example.

Our stability and uniqueness result requires only one measurement: $\mathcal{N}=1$. For the determination of the three coefficients by a single measurement, we have to choose initial data which satisfy stronger Conditions (3.11)(3.13) than in the case of $\mathcal{N} \geq 2$. Thus Conditions (3.11)-(3.13) are not generic properties and should be realized in a non-physical way by us. Moreover, as the following example shows, we can take $\mathbf{p}$ and $\mathbf{q}$ satisfying those.

Example of $\boldsymbol{\Omega}, \mathbf{p}, \mathbf{q}$ meeting (3.11) $-(\mathbf{3 . 1 3})$. We assume that $\lambda, \mu$ are positive constants and that $\left\{\left(x_{1}, x_{2}\right) \in\right.$ $\left.\bar{\Omega} ; x_{2}=y_{2}\right\}$ and $\left\{\left(x_{1}, x_{2}\right) \in \bar{\Omega} ; x_{1}=y_{1}\right\}$ are empty. Noting that the fourth columns of the matrices in (3.11) and (3.12) have $x^{\prime}-y^{\prime}$ as factors, we will take quadratic functions in $x^{\prime}$. For example, we take

$$
\mathbf{p}\left(x^{\prime}\right)=\left(\begin{array}{c}
0 \\
\left(x_{1}-y_{1}\right)\left(x_{2}-y_{2}\right)
\end{array}\right), \quad \mathbf{q}\left(x^{\prime}\right)=\left(\begin{array}{c}
\left(x_{2}-y_{2}\right)^{2} \\
0
\end{array}\right) .
$$

Then we can verify that (3.11)-(3.13) are all satisfied.

Remark 3.1. In place of (3.10), let us assume

$$
\Omega=\left\{\left(x_{1}, x_{2}\right) ; \widetilde{\gamma}_{0}\left(x_{1}\right)<x_{2}<\widetilde{\gamma}_{1}\left(x_{1}\right), x_{1} \in \widetilde{I}\right\}
$$

with some open interval $\widetilde{I}$. Then, after replacing (3.13) by

$$
\begin{aligned}
& x_{2}-y_{2} \neq 0, \\
& {\left[L_{\lambda, \mu} \mathbf{q}\right]_{2}\left(\partial_{x_{1}} p_{2}+\partial_{x_{2}} p_{1}\right)\left(x^{\prime}\right) \neq\left[L_{\lambda, \mu} \mathbf{p}\right]_{2}\left(\partial_{x_{1}} q_{2}+\partial_{x_{2}} q_{1}\right)\left(x^{\prime}\right), \quad x^{\prime} \in \bar{\Omega},}
\end{aligned}
$$

the conclusion of Theorem 3.1 holds under Conditions (3.11), (3.12) and (3.14). Moreover in the case when $\Omega$ is a more general smooth domain, we can prove the conditional stability in our inverse problem under other conditions on $\omega \subset \Omega$. We will omit the details, for the sake of compact description of the proof.

We set

$$
\psi(x)=\left|x^{\prime}-y^{\prime}\right|^{2}-\theta\left(x_{0}-\frac{T}{2}\right)^{2}, \quad \phi(x)=\mathrm{e}^{\tau \psi(x)}, \quad x=\left(x_{0}, x^{\prime}\right) \in Q .
$$


First we show

Lemma 3.1. Let $(\lambda, \mu, \rho) \in \mathcal{W}$, and let us assume (3.9) and (3.14). Then, for sufficiently large $\tau>0$, the function $\psi$ given by (3.15) satisfies Conditions 2.1 and (2.10). Therefore the conclusion of Theorem $2.3 \mathrm{holds}$ and the constants $C_{1}(\tau), \widehat{\tau}$ and $s_{0}(\tau)$ in (2.12) can be taken independently of $(\lambda, \mu, \rho) \in \mathcal{W}$.

Proof. Conditions (2.10) and the third condition in (2.6) are directly verified by means of (3.5). Conditions (2.4) and (2.5) can be verified by the same way as in Imanuvilov and Yamamoto [24], for example. Finally we have to verify the first and second conditions in (2.6). Without loss of generality, we may assume that $T=\frac{2 d}{\sqrt{\theta}}+\varepsilon$, where $\varepsilon>0$ is sufficiently small. Because if Theorem 3.1 is proved for this value of $T$, then the conclusion is true for any $\widetilde{T}>T$. Then, by noting that

$$
\left(\left|\frac{\partial \psi}{\partial \vec{\tau}}\right|^{2}+\left|\frac{\partial \psi}{\partial \vec{n}}\right|^{2}\right)^{\frac{1}{2}}=\left|\nabla_{x^{\prime}} \psi\right|
$$

and that the right hand side of the first inequality in (2.6) is greater than or equal to

$$
\min \left\{\frac{\mu\left(x^{\prime}\right)}{\sqrt{(\lambda+2 \mu)\left(x^{\prime}\right)}}, \frac{\sqrt{\mu(\lambda+\mu)\left(x^{\prime}\right)}}{\sqrt{(\lambda+2 \mu)\left(x^{\prime}\right)}}\right\}\left(\left|\frac{\partial \psi}{\partial \vec{\tau}}\right|^{2}+\left|\frac{\partial \psi}{\partial \vec{n}}\right|^{2}\right)^{\frac{1}{2}}
$$

in terms of (3.8), it suffices to verify

$$
-\left(\theta\left(x_{0}-T / 2\right)\right)^{2}+\theta_{1}\left|x^{\prime}-y^{\prime}\right|^{2}>0
$$

for $x \in[0, T] \times \partial \Omega$. In fact, by means of the second inequality in (3.9), we have

$$
\begin{aligned}
4 \theta_{1}\left|x^{\prime}-y^{\prime}\right|^{2}-4 \theta^{2}\left(x_{0}-\frac{T}{2}\right)^{2} & \geq 4 \theta_{1} \inf _{x^{\prime} \in \Omega}\left|x^{\prime}-y^{\prime}\right|^{2}-\theta\left(\theta T^{2}\right) \\
& \geq 4 \theta_{1} \inf _{x^{\prime} \in \Omega}\left|x^{\prime}-y^{\prime}\right|^{2}-\theta(2 d+\varepsilon \sqrt{\theta})^{2} \\
& >0
\end{aligned}
$$

because $\varepsilon>0$ is sufficiently small. The uniformity of the constants $C_{1}(\tau), \widehat{\tau}$ and $s_{0}(\tau)$ follows similarly to [19]. Thus the proof of Lemma 3.1 is complete.

Next we prove a Carleman estimate for a first order partial differential operator

$$
\left(P_{0} g\right)\left(x^{\prime}\right)=\sum_{j=1}^{2} p_{0, j}\left(x^{\prime}\right) \partial_{x_{j}} g\left(x^{\prime}\right)
$$

where $p_{0, j} \in C^{1}(\bar{\Omega}), j=1,2$.

Lemma 3.2. We assume

$$
\sum_{j=1}^{2} p_{0, j}\left(x^{\prime}\right) \partial_{x_{j}} \phi\left(T / 2, x^{\prime}\right)>0, \quad x^{\prime} \in \bar{\Omega} .
$$

Then there exists a constant $\tau_{0}>0$ such that for all $\tau>\tau_{0}$, there exist $s_{0}=s_{0}(\tau)>0$ and $C_{2}=C_{2}\left(s_{0}, \tau_{0}, \Omega, \omega\right)>$ 0 such that

$$
\int_{\Omega} s^{2}|g|^{2} \mathrm{e}^{2 s \phi\left(T / 2, x^{\prime}\right)} \mathrm{d} x^{\prime} \leq C_{2} \int_{\Omega}\left|P_{0} g\right|^{2} \mathrm{e}^{2 s \phi\left(T / 2, x^{\prime}\right)} \mathrm{d} x^{\prime}
$$


for all $s>s_{0}$ and $g \in H^{1}(\Omega)$ satisfying

$$
g=0 \quad \text { on }\left\{x^{\prime} \in \partial \Omega ; \sum_{j=1}^{2} p_{0, j}\left(x^{\prime}\right) n_{j}\left(x^{\prime}\right) \geq 0\right\} .
$$

Lemma 3.3. We assume

$$
\sum_{j=1}^{2} p_{0, j}\left(x^{\prime}\right) \partial_{x_{j}} \phi\left(T / 2, x^{\prime}\right) \neq 0, \quad x^{\prime} \in \bar{\Omega} .
$$

Then the conclusion of Lemma 3.2 is true for all $s>s_{0}$ and $g \in H_{0}^{1}(\Omega)$.

Proof of Lemma 3.2. For simplicity, we set $\phi_{0}\left(x^{\prime}\right)=\phi\left(T / 2, x^{\prime}\right)$ and $w=\mathrm{e}^{s \phi_{0}} g, Q_{0} w=\mathrm{e}^{s \phi_{0}} P_{0}\left(\mathrm{e}^{-s \phi_{0}} w\right)$. Then

$$
\int_{\Omega}\left|P_{0} g\right|^{2} \mathrm{e}^{2 s \phi\left(T / 2, x^{\prime}\right)} \mathrm{d} x^{\prime}=\int_{\Omega}\left|Q_{0} w\right|^{2} \mathrm{~d} x^{\prime} .
$$

We have

$$
Q_{0} w=P_{0} w-s q_{0} w
$$

where $q_{0}\left(x^{\prime}\right)=\sum_{j=1}^{2} p_{0, j}\left(x^{\prime}\right) \partial_{x_{j}} \phi_{0}\left(x^{\prime}\right)$. Therefore, by (3.16) and integration by parts, we obtain

$$
\begin{aligned}
\left\|Q_{0} w\right\|_{L^{2}(\Omega)}^{2} & =\left\|P_{0} w\right\|_{L^{2}(\Omega)}^{2}+s^{2}\left\|q_{0} w\right\|_{L^{2}(\Omega)}^{2}-2 s \int_{\Omega} \sum_{j=1}^{2} p_{0, j}\left(\partial_{x_{j}} w\right) q_{0} w \mathrm{~d} x^{\prime} \\
& \geq s^{2} \int_{\Omega} q_{0}\left(x^{\prime}\right)^{2} w^{2}\left(x^{\prime}\right) \mathrm{d} x^{\prime}-s \int_{\Omega} \sum_{j=1}^{2} p_{0, j} q_{0} \partial_{x_{j}}\left(w^{2}\right) \mathrm{d} x^{\prime} \\
& \geq C_{0} s^{2} \int_{\Omega} w^{2}\left(x^{\prime}\right) \mathrm{d} x^{\prime}-s \int_{\partial \Omega} \sum_{j=1}^{2} p_{0, j} q_{0} w^{2} n_{j} \mathrm{~d} S+s \int_{\Omega} \sum_{j=1}^{2} \partial_{x_{j}}\left(p_{0, j} q_{0}\right) w^{2} \mathrm{~d} x^{\prime} \\
& \geq\left(C_{2} s^{2}-C_{3} s\right) \int_{\Omega} w^{2} \mathrm{~d} x^{\prime}-s \int_{\partial \Omega \cap\left\{\sum_{j=1}^{2} p_{0, j} n_{j} \leq 0\right\}}^{2}\left(\sum_{j=1} p_{0, j} n_{j}\right) q_{0} w^{2} \mathrm{~d} S .
\end{aligned}
$$

By (3.16), we have $q_{0}>0$ on $\partial \Omega$, so that the right hand side is greater than or equal to $\left(C_{2} s^{2}-C_{3} s\right) \int_{\Omega} w^{2} \mathrm{~d} x^{\prime}$. Thus by taking $s>0$ sufficiently large, the proof of Lemma 3.2 is complete.

The proof of Lemma 3.3 is similar, thanks to the fact that the integral on $\partial \Omega$ vanishes for $g \in H_{0}^{1}(\Omega)$.

Now we proceed to

Proof of Theorem 3.1. The proof is similar to Isakov, Imanuvilov and Yamamoto [19], Imanuvilov and Yamamoto [22-24] and the new ingredient is an $H^{-1}$-Carleman estimate (Lem. 3.1) . Henceforth, for simplicity, we set

$$
\mathbf{u}=\mathbf{u}(\lambda, \mu, \rho, \mathbf{p}, \mathbf{q}, \eta), \quad \mathbf{v}=\mathbf{u}(\widetilde{\lambda}, \widetilde{\mu}, \widetilde{\rho}, \mathbf{p}, \mathbf{q}, \eta)
$$

and

$$
\mathbf{y}=\mathbf{u}-\mathbf{v}, \quad f=\rho-\widetilde{\rho}, \quad g=\lambda-\widetilde{\lambda}, \quad h=\mu-\widetilde{\mu} .
$$

In (3.13), without loss of generality, we may assume that

$$
x_{1}-y_{1}>0, \quad\left(x_{1}, x_{2}\right) \in \bar{\Omega} .
$$


Then we set

$$
F\left(x_{1}, x_{2}\right)=\int_{\gamma_{1}\left(x_{2}\right)}^{x_{1}} f\left(\xi, x_{2}\right) \mathrm{d} \xi, \quad\left(x_{1}, x_{2}\right) \in \Omega .
$$

If $x_{1}-y_{1}<0$ for $\left(x_{1}, x_{2}\right) \in \bar{\Omega}$, then it is sufficient to replace (3.17) by $F\left(x_{1}, x_{2}\right)=\int_{\gamma_{0}\left(x_{2}\right)}^{x_{1}} f\left(\xi, x_{2}\right) \mathrm{d} \xi,\left(x_{1}, x_{2}\right) \in \Omega$. Then

$$
\widetilde{\rho} \partial_{x_{0}}^{2} \mathbf{y}=L_{\widetilde{\lambda}, \widetilde{\mu}} \mathbf{y}+G \mathbf{u} \text { in } Q
$$

and

$$
\mathbf{y}\left(\frac{T}{2}, x^{\prime}\right)=\partial_{x_{0}} \mathbf{y}\left(\frac{T}{2}, x^{\prime}\right)=0, \quad x^{\prime} \in \Omega
$$

and

$$
\mathbf{y}=0 \quad \text { on }(0, T) \times \partial \Omega
$$

Here we set

$$
\begin{aligned}
G \mathbf{u}(x)=-\partial_{x_{1}} F\left(x^{\prime}\right) \partial_{x_{0}}^{2} \mathbf{u}(x)+(g+h)\left(x^{\prime}\right) \nabla_{x^{\prime}} & (\operatorname{div} \mathbf{u})(x)+h\left(x^{\prime}\right) \Delta \mathbf{u}(x) \\
& +(\operatorname{div} \mathbf{u})(x) \nabla_{x^{\prime}} g\left(x^{\prime}\right)+\left(\nabla_{x^{\prime}} \mathbf{u}(x)+\left(\nabla_{x^{\prime}} \mathbf{u}(x)\right)^{T}\right) \nabla_{x^{\prime}} h\left(x^{\prime}\right) .
\end{aligned}
$$

By (3.14), we have the inequality $\frac{\theta T^{2}}{4}>d^{2}$. Therefore, by (3.6) and Definition (3.15) of the function $\phi$, we have

$$
\phi\left(T / 2, x^{\prime}\right) \geq d_{1}, \quad \phi\left(0, x^{\prime}\right)=\phi\left(T, x^{\prime}\right)<d_{1}, \quad x^{\prime} \in \bar{\Omega}
$$

with $d_{1}=\exp \left(\tau \inf _{x^{\prime} \in \Omega}\left|x^{\prime}-y^{\prime}\right|^{2}\right)$. Thus, for given $\varepsilon>0$, we can choose a sufficiently small $\delta=\delta(\varepsilon)>0$ such that

$$
\phi(x) \geq d_{1}-\varepsilon, \quad x \in\left[\frac{T}{2}-\delta, \frac{T}{2}+\delta\right] \times \bar{\Omega}
$$

and

$$
\phi(x) \leq d_{1}-2 \varepsilon, \quad x \in([0,2 \delta] \cup[T-2 \delta, T]) \times \bar{\Omega} .
$$

In order to apply Lemma 3.1, it is necessary to introduce a cut-off function $\chi$ satisfying $0 \leq \chi \leq 1, \chi \in C^{\infty}(\mathbb{R})$ and

$$
\chi= \begin{cases}0 & \text { on }[0, \delta] \cup[T-\delta, T] \\ 1 & \text { on }[2 \delta, T-2 \delta] .\end{cases}
$$

In the sequel, $C_{j}>0$ denote generic constants depending on $s_{0}, \tau, M_{0}, M_{1}, \theta_{0}, \theta_{1}, \eta, \Omega, T, y^{\prime}, \omega, \chi$ and $\mathbf{p}, \mathbf{q}$, $\varepsilon, \delta$, but independent of $s>s_{0}$.

Setting $\mathbf{z}_{1}=\chi \partial_{x_{0}}^{2} \mathbf{y}, \mathbf{z}_{2}=\chi \partial_{x_{0}}^{3} \mathbf{y}$ and $\mathbf{z}_{3}=\chi \partial_{x_{0}}^{4} \mathbf{y}$, we have

$$
\left\{\begin{array}{l}
\widetilde{\rho} \partial_{x_{0}}^{2} \mathbf{z}_{1}=L_{\widetilde{\lambda}, \widetilde{\mu}} \mathbf{z}_{1}+\chi G\left(\partial_{x_{0}}^{2} \mathbf{u}\right)+2 \widetilde{\rho}\left(\partial_{x_{0}} \chi\right) \partial_{x_{0}}^{3} \mathbf{y}+\widetilde{\rho}\left(\partial_{x_{0}}^{2} \chi\right) \partial_{x_{0}}^{2} \mathbf{y} \\
\widetilde{\rho} \partial_{x_{0}}^{2} \mathbf{z}_{2}=L_{\widetilde{\lambda}, \widetilde{\mu}} \mathbf{z}_{2}+\chi G\left(\partial_{x_{0}}^{3} \mathbf{u}\right)+2 \widetilde{\rho}\left(\partial_{x_{0}} \chi\right) \partial_{x_{0}}^{4} \mathbf{y}+\widetilde{\rho}\left(\partial_{x_{0}}^{2} \chi\right) \partial_{x_{0}}^{3} \mathbf{y} \\
\widetilde{\rho} \partial_{x_{0}}^{2} \mathbf{z}_{3}=L_{\widetilde{\lambda}, \widetilde{\mu}} \mathbf{z}_{3}+\chi G\left(\partial_{x_{0}}^{4} \mathbf{u}\right)+2 \widetilde{\rho}\left(\partial_{x_{0}} \chi\right) \partial_{x_{0}}^{5} \mathbf{y}+\widetilde{\rho}\left(\partial_{x_{0}}^{2} \chi\right) \partial_{x_{0}}^{4} \mathbf{y} \quad \text { in } Q .
\end{array}\right.
$$

Henceforth we set

$$
\mathcal{E}=\int_{Q_{\omega}}\left(\left|\partial_{x_{0}}^{2} \mathbf{y}\right|^{2}+\left|\partial_{x_{0}}^{3} \mathbf{y}\right|^{2}+\left|\partial_{x_{0}}^{4} \mathbf{y}\right|^{2}\right) \mathrm{e}^{2 s \phi} \mathrm{d} x
$$


Noting that $\mathbf{u} \in W^{7, \infty}(Q)$, in view of (3.24) and Lemma 3.1, we can apply Theorem 2.3 to (3.25), so that

$$
\begin{aligned}
& \sum_{j=2}^{4} \int_{Q}\left|\partial_{x_{0}}^{j} \mathbf{y}\right|^{2} \chi^{2} \mathrm{e}^{2 s \phi} \mathrm{d} x \leq C_{5}\left(\left\|F \mathrm{e}^{s \phi}\right\|_{L^{2}(Q)}^{2}+\left\|g \mathrm{e}^{s \phi}\right\|_{L^{2}(Q)}^{2}+\left\|h \mathrm{e}^{s \phi}\right\|_{L^{2}(Q)}^{2}\right) \\
& +C_{5} \sum_{j=3}^{5}\left\|\left(\partial_{x_{0}} \chi\right)\left(\partial_{x_{0}}^{j} \mathbf{y}\right) \mathrm{e}^{s \phi}\right\|_{L^{2}\left(0, T ;\left(H^{-1}(\Omega)\right)^{2}\right)}^{2} \\
& +C_{5} \sum_{j=2}^{4}\left\|\left(\partial_{x_{0}}^{2} \chi\right)\left(\partial_{x_{0}}^{j} \mathbf{y}\right) \mathrm{e}^{s \phi}\right\|_{L^{2}\left(0, T ;\left(H^{-1}(\Omega)\right)^{2}\right)}^{2}+C_{5} \mathcal{E} \\
& \leq C_{6}\left(\left\|F \mathrm{e}^{s \phi}\right\|_{L^{2}(Q)}^{2}+\left\|g \mathrm{e}^{s \phi}\right\|_{L^{2}(Q)}^{2}+\left\|h \mathrm{e}^{s \phi}\right\|_{L^{2}(Q)}^{2}\right)+C_{6} \mathrm{e}^{2 s\left(d_{1}-2 \varepsilon\right)}+C_{7} \mathcal{E}
\end{aligned}
$$

for all large $s>0$

On the other hand,

$$
\begin{aligned}
& \int_{\Omega}\left|\left(\partial_{x_{0}}^{2} \mathbf{y}\right)\left(T / 2, x^{\prime}\right)\right|^{2} \mathrm{e}^{2 s \phi\left(T / 2, x^{\prime}\right)} \mathrm{d} x^{\prime} \\
& =\int_{0}^{T / 2} \frac{\partial}{\partial x_{0}}\left(\int_{\Omega}\left|\left(\partial_{x_{0}}^{2} \mathbf{y}\right)\left(x_{0}, x^{\prime}\right)\right|^{2} \chi\left(x_{0}\right)^{2} \mathrm{e}^{2 s \phi} \mathrm{d} x^{\prime}\right) \mathrm{d} x_{0} \\
& =\int_{0}^{T / 2} \int_{\Omega} 2\left(\left(\partial_{x_{0}}^{3} \mathbf{y}\right) \cdot\left(\partial_{x_{0}}^{2} \mathbf{y}\right)\right) \chi^{2} \mathrm{e}^{2 s \phi} \mathrm{d} x \\
& +2 s \int_{0}^{T / 2} \int_{\Omega}\left|\partial_{x_{0}}^{2} \mathbf{y}\right|^{2} \chi^{2}\left(\partial_{x_{0}} \phi\right) \mathrm{e}^{2 s \phi} \mathrm{d} x+\int_{0}^{T / 2} \int_{\Omega}\left|\partial_{x_{0}}^{2} \mathbf{y}\right|^{2}\left(\partial_{x_{0}}\left(\chi^{2}\right)\right) \mathrm{e}^{2 s \phi} \mathrm{d} x \\
& \leq C_{7} \int_{Q} s \chi^{2}\left(\left|\partial_{x_{0}}^{3} \mathbf{y}\right|^{2}+\left|\partial_{x_{0}}^{2} \mathbf{y}\right|^{2}\right) \mathrm{e}^{2 s \phi} \mathrm{d} x+C_{7} \mathrm{e}^{2 s\left(d_{1}-2 \varepsilon\right)} \text {. }
\end{aligned}
$$

Therefore (3.26) yields

$$
\int_{\Omega}\left|\left(\partial_{x_{0}}^{2} \mathbf{y}\right)\left(T / 2, x^{\prime}\right)\right|^{2} \mathrm{e}^{2 s \phi\left(T / 2, x^{\prime}\right)} \mathrm{d} x^{\prime} \leq C_{8} s \int_{Q}\left(|F|^{2}+|g|^{2}+|h|^{2}\right) \mathrm{e}^{2 s \phi} \mathrm{d} x+C_{8} s \mathrm{e}^{2 s\left(d_{1}-2 \varepsilon\right)}+C_{8} s \mathcal{E}
$$

for all large $s>0$. Similarly we can estimate $\int_{\Omega}\left|\left(\partial_{x_{0}}^{3} \mathbf{y}\right)\left(T / 2, x^{\prime}\right)\right|^{2} \mathrm{e}^{2 s \phi\left(T / 2, x^{\prime}\right)} \mathrm{d} x^{\prime}$ to obtain

$$
\begin{aligned}
& \int_{\Omega}\left(\left|\left(\partial_{x_{0}}^{2} \mathbf{y}\right)\left(T / 2, x^{\prime}\right)\right|^{2}+\left|\left(\partial_{x_{0}}^{3} \mathbf{y}\right)\left(T / 2, x^{\prime}\right)\right|^{2}\right) \mathrm{e}^{2 s \phi\left(T / 2, x^{\prime}\right)} \mathrm{d} x^{\prime} \\
& \leq C_{9} s \int_{Q}\left(|F|^{2}+|g|^{2}+|h|^{2}\right) \mathrm{e}^{2 s \phi} \mathrm{d} x+C_{9} s \mathrm{e}^{2 s\left(d_{1}-2 \varepsilon\right)}+C_{9} s \mathcal{E}
\end{aligned}
$$

for all large $s>0$.

Now first order partial differential equations satisfied by $h, g$ and $F$ are going to be considered. That is, by (3.18), (3.19) and $\mathbf{u}, \mathbf{v} \in W^{7, \infty}(Q)$, we have

$$
\widetilde{\rho} \partial_{x_{0}}^{2} \mathbf{y}\left(\frac{T}{2}, x^{\prime}\right)=G \mathbf{u}\left(\frac{T}{2}, x^{\prime}\right), \quad \widetilde{\rho} \partial_{x_{0}}^{3} \mathbf{y}\left(\frac{T}{2}, x^{\prime}\right)=G \partial_{x_{0}} \mathbf{u}\left(\frac{T}{2}, x^{\prime}\right)
$$


Then, setting

we rewrite $(3.29)$ as

$$
\left\{\begin{array}{l}
-\frac{1}{\rho} L_{\lambda, \mu} \mathbf{p}=\left(\begin{array}{c}
a_{11} \\
a_{21}
\end{array}\right), \quad-\frac{1}{\rho} L_{\lambda, \mu} \mathbf{q}=\left(\begin{array}{c}
a_{12} \\
a_{22}
\end{array}\right), \\
\operatorname{div} \mathbf{p}=b_{1}, \quad \operatorname{div} \mathbf{q}=b_{2}, \\
\nabla_{x^{\prime}} \mathbf{p}+\left(\nabla_{x^{\prime}} \mathbf{p}\right)^{T}=\left(\begin{array}{ll}
c_{1} & d_{1} \\
d_{1} & e_{1}
\end{array}\right), \quad \nabla_{x^{\prime}} \mathbf{q}+\left(\nabla_{x^{\prime}} \mathbf{q}\right)^{T}=\left(\begin{array}{ll}
c_{2} & d_{2} \\
d_{2} & e_{2}
\end{array}\right), \\
\widetilde{\rho} \partial_{x_{0}}^{2} \mathbf{y}\left(\frac{T}{2}, x^{\prime}\right)-(g+h) \nabla_{x^{\prime}}(\operatorname{div} \mathbf{p})-h \Delta \mathbf{p}=\left(\begin{array}{c}
G_{11} \\
G_{21}
\end{array}\right), \\
\widetilde{\rho} \partial_{x_{0}}^{3} \mathbf{y}\left(\frac{T}{2}, x^{\prime}\right)-(g+h) \nabla_{x^{\prime}}(\operatorname{div} \mathbf{q})-h \Delta \mathbf{q}=\left(\begin{array}{c}
G_{12} \\
G_{22}
\end{array}\right),
\end{array}\right.
$$

$$
\left(\begin{array}{ccc}
a_{11} & b_{1} & 0 \\
a_{21} & 0 & b_{1} \\
a_{12} & b_{2} & 0 \\
a_{22} & 0 & b_{2}
\end{array}\right)\left(\begin{array}{l}
\partial_{x_{1}} F \\
\partial_{x_{1}} g \\
\partial_{x_{2}} g
\end{array}\right)=\left(\begin{array}{l}
G_{11}-c_{1} \partial_{x_{1}} h-d_{1} \partial_{x_{2}} h \\
G_{21}-d_{1} \partial_{x_{1}} h-e_{1} \partial_{x_{2}} h \\
G_{12}-c_{2} \partial_{x_{1}} h-d_{2} \partial_{x_{2}} h \\
G_{22}-d_{2} \partial_{x_{1}} h-e_{2} \partial_{x_{2}} h
\end{array}\right)
$$

Because linear system (3.31) possesses a solution $\left(\partial_{x_{1}} F, \partial_{x_{1}} g, \partial_{x_{2}} g\right)$, the coefficient matrix must satisfy

$$
\operatorname{det}\left(\begin{array}{cccc}
a_{11} & b_{1} & 0 & G_{11}-c_{1} \partial_{x_{1}} h-d_{1} \partial_{x_{2}} h \\
a_{21} & 0 & b_{1} & G_{21}-d_{1} \partial_{x_{1}} h-e_{1} \partial_{x_{2}} h \\
a_{12} & b_{2} & 0 & G_{12}-c_{2} \partial_{x_{1}} h-d_{2} \partial_{x_{2}} h \\
a_{22} & 0 & b_{2} & G_{22}-d_{2} \partial_{x_{1}} h-e_{2} \partial_{x_{2}} h
\end{array}\right)=0
$$

that is,

$$
\left(\partial_{x_{1}} h\right) \operatorname{det}\left(\begin{array}{cccc}
a_{11} & b_{1} & 0 & c_{1} \\
a_{21} & 0 & b_{1} & d_{1} \\
a_{12} & b_{2} & 0 & c_{2} \\
a_{22} & 0 & b_{2} & d_{2}
\end{array}\right)+\left(\partial_{x_{2}} h\right) \operatorname{det}\left(\begin{array}{cccc}
a_{11} & b_{1} & 0 & d_{1} \\
a_{21} & 0 & b_{1} & e_{1} \\
a_{12} & b_{2} & 0 & d_{2} \\
a_{22} & 0 & b_{2} & e_{2}
\end{array}\right)=\operatorname{det}\left(\begin{array}{cccc}
a_{11} & b_{1} & 0 & G_{11} \\
a_{21} & 0 & b_{1} & G_{21} \\
a_{12} & b_{2} & 0 & G_{12} \\
a_{22} & 0 & b_{2} & G_{22}
\end{array}\right)
$$

by the linearity of the determinant. Under Condition (3.11), taking into consideration $h=\mu-\widetilde{\mu}=0$ on $\partial \Omega$ and considering (3.32) as a first order partial differential operator in $h$, we apply Lemma 3.3, so that

$$
\begin{aligned}
s^{2} \int_{\Omega}|h|^{2} e^{2 s \phi\left(T / 2, x^{\prime}\right)} \mathrm{d} x^{\prime} & \leq C_{10}\left\|\operatorname{det}\left(\begin{array}{cccc}
a_{11} & b_{1} & 0 & G_{11} \\
a_{21} & 0 & b_{1} & G_{21} \\
a_{12} & b_{2} & 0 & G_{12} \\
a_{22} & 0 & b_{2} & G_{22}
\end{array}\right) \mathrm{e}^{s \phi(T / 2, \cdot)}\right\|_{L^{2}(\Omega)}^{2} \\
& \leq C_{11} \int_{\Omega}\left(\left|\partial_{x_{0}}^{2} \mathbf{y}\left(\frac{T}{2}, x^{\prime}\right)\right|^{2}+\left|\partial_{x_{0}}^{3} \mathbf{y}\left(\frac{T}{2}, x^{\prime}\right)\right|^{2}\right) \mathrm{e}^{2 s \phi\left(T / 2, x^{\prime}\right)} \mathrm{d} x^{\prime} \\
& +C_{11} \int_{\Omega}\left(|g|^{2}+|h|^{2}\right) e^{2 s \phi\left(T / 2, x^{\prime}\right)} \mathrm{d} x^{\prime}
\end{aligned}
$$

in view of (3.30). We rewrite (3.29) as

$$
\left(\begin{array}{lll}
a_{11} & c_{1} & d_{1} \\
a_{21} & d_{1} & e_{1} \\
a_{12} & c_{2} & d_{2} \\
a_{22} & d_{2} & e_{2}
\end{array}\right)\left(\begin{array}{l}
\partial_{x_{1}} F \\
\partial_{x_{1}} h \\
\partial_{x_{2}} h
\end{array}\right)=\left(\begin{array}{l}
G_{11}-b_{1} \partial_{x_{1}} g \\
G_{21}-b_{1} \partial_{x_{2}} g \\
G_{12}-b_{2} \partial_{x_{1}} g \\
G_{22}-b_{2} \partial_{x_{2}} g
\end{array}\right)
$$


and, using (3.12), we can similarly deduce

$$
\begin{aligned}
s^{2} \int_{\Omega}|g|^{2} \mathrm{e}^{2 s \phi\left(T / 2, x^{\prime}\right)} \mathrm{d} x^{\prime} \leq C_{12} \int_{\Omega}\left(\left|\partial_{x_{0}}^{2} \mathbf{y}\left(\frac{T}{2}, x^{\prime}\right)\right|^{2}\right. & \left.+\left|\partial_{x_{0}}^{3} \mathbf{y}\left(\frac{T}{2}, x^{\prime}\right)\right|^{2}\right) \mathrm{e}^{2 s \phi\left(T / 2, x^{\prime}\right)} \mathrm{d} x^{\prime} \\
& +C_{12} \int_{\Omega}\left(|g|^{2}+|h|^{2}\right) e^{2 s \phi\left(T / 2, x^{\prime}\right)} \mathrm{d} x^{\prime}
\end{aligned}
$$

for all large $s>0$. By (3.33) and (3.34), for sufficiently large $s>0$, we have

$$
s^{2} \int_{\Omega}\left(|g|^{2}+|h|^{2}\right) \mathrm{e}^{2 s \phi\left(T / 2, x^{\prime}\right)} \mathrm{d} x^{\prime} \leq C_{13} \int_{\Omega}\left(\left|\partial_{x_{0}}^{2} \mathbf{y}\left(\frac{T}{2}, x^{\prime}\right)\right|^{2}+\left|\partial_{x_{0}}^{3} \mathbf{y}\left(\frac{T}{2}, x^{\prime}\right)\right|^{2}\right) \mathrm{e}^{2 s \phi\left(T / 2, x^{\prime}\right)} \mathrm{d} x^{\prime}
$$

Moreover, eliminating $\partial_{x_{2}} h$ in the first and the third rows in (3.31) and using (3.13), we have

$$
\begin{aligned}
\partial_{x_{1}}\left(F+\frac{d_{2} b_{1}-d_{1} b_{2}}{d_{2} a_{11}-d_{1} a_{12}} g+\frac{d_{2} c_{1}-d_{1} c_{2}}{d_{2} a_{11}-d_{1} a_{12}} h\right) & \\
& =\frac{d_{2} G_{11}-d_{1} G_{12}}{d_{2} a_{11}-d_{1} a_{12}}+g \partial_{x_{1}}\left(\frac{d_{2} b_{1}-d_{1} b_{2}}{d_{2} a_{11}-d_{1} a_{12}}\right)+h \partial_{x_{1}}\left(\frac{d_{2} c_{1}-d_{1} c_{2}}{d_{2} a_{11}-d_{1} a_{12}}\right) .
\end{aligned}
$$

By (3.10) and (3.17), if $n_{1}\left(x^{\prime}\right) \geq 0$, then $x_{1}=\gamma_{1}\left(x_{2}\right)$, that is, we have: $F\left(x_{1}, x_{2}\right)=0$ for $n_{1}\left(x^{\prime}\right) \geq 0$. Therefore, noting $g=h=0$ on $\partial \Omega$ and setting $p_{0,1}=1, p_{0,2}=0$ in Lemma 3.2, we can apply the lemma. Thus, in view of (3.35) and (3.30), we obtain

$$
s^{2} \int_{\Omega}|F|^{2} \mathrm{e}^{2 s \phi\left(T / 2, x^{\prime}\right)} \mathrm{d} x^{\prime} \leq C_{14} \int_{\Omega}\left(\left|\partial_{x_{0}}^{2} \mathbf{y}\left(\frac{T}{2}, x^{\prime}\right)\right|^{2}+\left|\partial_{x_{0}}^{3} \mathbf{y}\left(\frac{T}{2}, x^{\prime}\right)\right|^{2}\right) \mathrm{e}^{2 s \phi\left(T / 2, x^{\prime}\right)} \mathrm{d} x^{\prime}
$$

for all large $s>0$. Consequently, substituting (3.35) and (3.36) into (3.28) and using $\phi\left(T / 2, x^{\prime}\right) \geq \phi\left(x_{0}, x^{\prime}\right)$ for $\left(x_{0}, x^{\prime}\right) \in Q$, we obtain

$$
\int_{\Omega}\left(|F|^{2}+|g|^{2}+|h|^{2}\right) \mathrm{e}^{2 s \phi\left(T / 2, x^{\prime}\right)} \mathrm{d} x^{\prime} \leq \frac{C_{15} T}{s} \int_{\Omega}\left(|F|^{2}+|g|^{2}+|h|^{2}\right) \mathrm{e}^{2 s \phi\left(T / 2, x^{\prime}\right)} \mathrm{d} x^{\prime}+\frac{C_{15}}{s} \mathrm{e}^{2 s\left(d_{1}-2 \varepsilon\right)}+\frac{C_{15}}{s} \mathcal{E}
$$

for all large $s>0$. Taking $s>0$ sufficiently large and noting $\mathrm{e}^{2 s \phi\left(T / 2, x^{\prime}\right)} \geq \mathrm{e}^{2 s d_{1}}$ for $x^{\prime} \in \bar{\Omega}$, we obtain

$$
\int_{\Omega}\left(|F|^{2}+|g|^{2}+|h|^{2}\right) \mathrm{d} x^{\prime} \leq C_{16} \mathrm{e}^{-4 s \varepsilon}+C_{17} \mathrm{e}^{2 s C_{18}} \mathcal{E}
$$

for all large $s>s_{0}$ : a constant which is dependent on $\tau$, but independent of $s$. Next we take in (3.37) instead of the constant $C_{17}$ the constant $C_{17} e^{2 s_{0} C_{18}}$. Now this inequality holds true for all $s>0$.

Now we choose $s>0$ such that

$$
\mathrm{e}^{2 s C_{16}} \mathcal{E}=\mathrm{e}^{-4 s \varepsilon}
$$

that is,

$$
s=-\frac{1}{4 \varepsilon+2 C_{16}} \ln \mathcal{E} .
$$

Here we may assume that $\mathcal{E}<1$ and so $s>0$. Then it follows from (3.37) that

$$
\int_{\Omega}\left(|F|^{2}+|g|^{2}+|h|^{2}\right) \mathrm{d} x^{\prime} \leq 2 C \mathcal{E}^{\frac{4 \varepsilon}{4 \varepsilon+2 C}}
$$


By Definition (3.17) of $F$, we have

$$
\int_{\Omega} f r \mathrm{~d} x_{1} \mathrm{~d} x_{2}=\int_{\Omega}\left(\partial_{x_{1}} F\right) r \mathrm{~d} x_{1} \mathrm{~d} x_{2}=\int_{\Omega} F\left(\partial_{x_{1}} r\right) \mathrm{d} x_{1} \mathrm{~d} x_{2}
$$

for all $r \in H_{0}^{1}(\Omega)$ by integration by parts. Hence we can directly verify that $\|f\|_{H^{-1}(\Omega)} \leq C\|F\|_{L^{2}(\Omega)}$, so that the proof of Theorem 3.1 is complete.

\section{Proof of Theorem 2.1}

Without loss of generality, we may assume that $\rho \equiv 1$. Otherwise we introduce new coefficients $\mu_{1}=$ $\mu / \rho, \lambda_{1}=\lambda / \rho$ to argue similarly. We can directly verify that the functions $\operatorname{rot} \mathbf{u} \equiv \partial_{x_{1}} u_{2}-\partial_{x_{2}} u_{1}$ and $\operatorname{div} \mathbf{u}$ satisfy the equations

$$
\partial_{x_{0}}^{2} \operatorname{rot} \mathbf{u}-\mu \Delta \operatorname{rot} \mathbf{u}=m_{1}, \quad \partial_{x_{0}}^{2} \operatorname{div} \mathbf{u}-(\lambda+2 \mu) \Delta \operatorname{div} \mathbf{u}=m_{2} \quad \text { in } Q,
$$

where

$$
m_{1}=K_{1} \operatorname{rot} \mathbf{u}+K_{2} \operatorname{div} \mathbf{u}+\mathcal{K}_{1} \mathbf{u}+\operatorname{rot} \mathbf{f}, \quad m_{2}=K_{3} \operatorname{rot} \mathbf{u}+K_{4} \operatorname{div} \mathbf{u}+\mathcal{K}_{2} \mathbf{u}+\operatorname{div} \mathbf{f}
$$

and $K_{j}, \mathcal{K}_{k}$ are first order differential operators with $L^{\infty}$ coefficients.

Thanks to Condition 2.1 on the weight function $\psi$, there exists $\widehat{\tau}$ such that for all $\tau>\widehat{\tau}$, the Carleman estimate for equations (4.1) (see e.g., [45]) yields the inequality

$$
\begin{aligned}
s\left\|(\nabla \operatorname{rot} \mathbf{u}) \mathrm{e}^{s \phi}\right\|_{\left(L^{2}(Q)\right)^{2}}^{2} & +s\left\|(\nabla \operatorname{div} \mathbf{u}) \mathrm{e}^{s \phi}\right\|_{\left(L^{2}(Q)\right)^{2}}^{2}+s^{3}\left\|(\operatorname{rot} \mathbf{u}) \mathrm{e}^{s \phi}\right\|_{\left(L^{2}(Q)\right)^{2}}^{2}+s^{3}\left\|(\operatorname{div} \mathbf{u}) e^{s \phi}\right\|_{\left(L^{2}(Q)\right)^{2}}^{2} \\
& \leq C_{1}\left(s^{2}\left\|\mathrm{fe}^{s \phi}\right\|_{\left(L^{2}(Q)\right)^{2}}^{2}+\left\|(\nabla \mathbf{f}) \mathrm{e}^{s \phi}\right\|_{\left(L^{2}(Q)\right)^{2}}^{2}+s\left\|\frac{\partial \mathbf{u}}{\partial \vec{n}} \mathrm{e}^{s \phi}\right\|_{\left(H^{1}((0, T) \times \partial \Omega)\right)^{2}}^{2}\right. \\
& \left.+s\left\|\frac{\partial^{2} \mathbf{u}}{\partial \vec{n}^{2}} \mathrm{e}^{s \phi}\right\|_{\left(L^{2}((0, T) \times \partial \Omega)\right)^{2}}^{2}+s^{3}\left\|\frac{\partial \mathbf{u}}{\partial \vec{n}} \mathrm{e}^{s \phi}\right\|_{\left(L^{2}((0, T) \times \partial \Omega)\right)^{2}}^{2}+\|\mathbf{u}\|_{\mathcal{B}\left(Q_{\omega}\right)}^{2}\right), \quad \forall s \geq s_{0}(\tau),
\end{aligned}
$$

where the constant $C_{1}$ is independent of $s$.

In order to estimate the $H^{1}(Q)$-norm of the function $\mathbf{u}$, we need the following proposition.

Proposition 4.1. There exists $\widehat{\tau}>1$ such that for any $\tau>\widehat{\tau}$, there exists $s_{0}(\tau)$ such that

$$
\begin{aligned}
& \int_{Q}\left(\frac{1}{s} \sum_{j, k=1}^{2}\left|\partial_{x_{j}} \partial_{x_{k}} \mathbf{u}\right|^{2}+s\left|\nabla_{x^{\prime}} \mathbf{u}\right|^{2}+s^{3}|\mathbf{u}|^{2}\right) \mathrm{e}^{2 s \phi} \mathrm{d} x \\
& \leq C_{2}\left(\left\|(\operatorname{rot} \mathbf{u}) \mathrm{e}^{s \phi}\right\|_{H^{1}(Q)}^{2}+\left\|(\operatorname{div} \mathbf{u}) \mathrm{e}^{s \phi}\right\|_{H^{1}(Q)}^{2}+\int_{Q_{\omega}}\left(s|\nabla \mathbf{u}|^{2}+s^{3}|\mathbf{u}|^{2}\right) \mathrm{e}^{2 s \phi} \mathrm{d} x\right), \\
& \quad \forall s \geq s_{0}(\tau), \mathbf{u} \in\left(H_{0}^{1}(Q)\right)^{2} .
\end{aligned}
$$

Proof of Proposition 4.1. Denote $\operatorname{rot} \mathbf{u}=\mathbf{y}$ and $\operatorname{div} \mathbf{u}=\mathbf{w}$ and let $\operatorname{rot}^{*} v=\left(\frac{\partial v}{\partial x_{2}},-\frac{\partial v}{\partial x_{1}}\right)$. Using a well-known formula: $\operatorname{rot}^{*} \operatorname{rot}=-\Delta_{x^{\prime}}+\nabla_{x^{\prime}}$ div, we obtain

$$
-\Delta_{x^{\prime}} \mathbf{u}=-\operatorname{rot}^{*} \mathbf{y}-\nabla_{x^{\prime}} \mathbf{w} \quad \text { in } \Omega,\left.\quad \mathbf{u}\right|_{\partial \Omega}=0 .
$$

Then (4.3) follows from the Carleman estimate for an elliptic equations obtained by the first author in [17]. 
By (4.2) and (4.3), we estimate $\sum_{|\alpha|=0, \alpha=\left(0, \alpha_{1}, \alpha_{2}\right)}^{2}\left\|\left(\partial_{x}^{\alpha} \mathbf{u}\right) \mathrm{e}^{s \phi}\right\|_{\left(L^{2}(Q)\right)^{2}}^{2}$ via the right hand side of inequality (4.2). Next using this estimate and equation (1.1), we obtain the estimate for the norm $\left\|\left(\partial_{x_{0}}^{2} \mathbf{u}\right) \mathrm{e}^{s \phi}\right\|_{\left(L^{2}(Q)\right)^{2}}^{2}$ via the right hand side of (4.2). Finally we obtain the estimate for $\left\|\left(\partial_{x_{0}} \partial_{x_{j}} \mathbf{u}\right) \mathrm{e}^{s \phi}\right\|_{\left(L^{2}(Q)\right)^{2}}^{2}$ and $s^{2}\left\|\left(\partial_{x_{0}} \mathbf{u}\right) \mathrm{e}^{s \phi}\right\|_{\left(L^{2}(Q)\right)^{2}}^{2}$ by the interpolation argument. Therefore, combining these estimates with (4.2), we have

$$
\begin{aligned}
\|\mathbf{u}\|_{Y(\phi, Q)}^{2} \leq & C_{3}\left(s^{2}\left\|\mathbf{f} \mathrm{e}^{s \phi}\right\|_{\left(L^{2}(Q)\right)^{2}}^{2}+\left\|(\nabla \mathbf{f}) \mathrm{e}^{s \phi}\right\|_{\left(L^{2}(Q)\right)^{2}}^{2}+s\left\|\frac{\partial^{2} \mathbf{u}}{\partial \vec{n}^{2}} \mathrm{e}^{s \phi}\right\|_{\left(L^{2}((0, T) \times \partial \Omega)\right)^{2}}^{2}\right. \\
& \left.+s\left\|\frac{\partial \mathbf{u}}{\partial \vec{n}} \mathrm{e}^{s \phi}\right\|_{\left(H^{1}((0, T) \times \partial \Omega)\right)^{2}}^{2}+\|\mathbf{u}\|_{\mathcal{B}\left(\phi, Q_{\omega}\right)}^{2}\right), \quad \forall s \geq s_{0}(\tau), \\
& +s^{3}\left\|\frac{\partial \mathbf{u}}{\partial \vec{n}} \mathrm{e}^{s \phi}\right\|_{\left(L^{2}((0, T) \times \partial \Omega)\right)^{2}}^{2},
\end{aligned}
$$

where the constant $C_{3}$ is independent of $s$. Here we recall definition (2.8) of $\|\mathbf{u}\|_{\mathcal{B}\left(\phi, Q_{\omega}\right)}^{2}$ and the definition of $\|\mathbf{u}\|_{Y(\phi, Q)}^{2}$ in $(2.9)$.

Now we need to estimate the boundary integrals at the right hand side of (4.4). In order to do that, it is convenient to use another weight function $\varphi$ such that $\left.\varphi\right|_{\partial \Omega}=\left.\phi\right|_{\partial \Omega}$ and $\varphi(x)<\phi(x)$ for all $x$ in small neighbourhood of $(0, T) \times \partial \Omega$. We introduce the function $\varphi$ by formulae:

$$
\varphi(x)=\mathrm{e}^{\tau \widetilde{\psi}(x)}, \quad \widetilde{\psi}(x)=\psi(x)-\widehat{\epsilon} \ell_{1}\left(x^{\prime}\right)+N \ell_{1}^{2}\left(x^{\prime}\right),
$$

where $\widehat{\epsilon}>0$ is a small positive parameter, $N>0$ is a large positive parameter, and $\ell_{1} \in C^{3}(\bar{\Omega})$ is a function such that

$$
\ell_{1}\left(x^{\prime}\right)>0, \quad \forall x^{\prime} \in \Omega,\left.\quad \ell_{1}\right|_{\partial \Omega}=0,\left.\quad \nabla_{x^{\prime}} \ell_{1}\right|_{\partial \Omega} \neq 0 .
$$

Denote $\Omega_{1 / N^{2}}=\left\{x^{\prime} \in \Omega\right.$; dist $\left.\left(x^{\prime}, \partial \Omega\right) \leq \frac{1}{N^{2}}\right\}$. Obviously for any fixed $\widehat{\epsilon}>0$, there exists $N_{0}(\widehat{\epsilon})$ such that

$$
\varphi(x)<\phi(x), \quad \forall x \in[0, T] \times \Omega_{1 / N^{2}}, \quad N \in\left(N_{0}(\widehat{\epsilon}), \infty\right) .
$$

Now we will prove the following estimate:

Lemma 4.1. Under conditions of Theorem 2.1, there exist $\widehat{\tau}>0$ and $N_{0}>1$ such that for all $\tau>\widehat{\tau}$, there exists $s_{0}(\tau, N)$ such that

$$
\begin{aligned}
\|\mathbf{u}\|_{Y(\varphi, Q)}^{2}+N & \sum_{|\alpha|=0}^{2} s^{4-2|\alpha|}\left\|\left(\partial_{x}^{\alpha} \mathbf{u}\right) \mathrm{e}^{s \varphi}\right\|_{\left(L^{2}(Q)\right)^{2}}^{2} \leq C_{4}\left(s^{2}\left\|\mathbf{f} \mathrm{e}^{s \varphi}\right\|_{\left(L^{2}(Q)\right)^{2}}^{2}\right. \\
& \left.+\left\|(\nabla \mathbf{f}) \mathrm{e}^{s \varphi}\right\|_{\left(L^{2}(Q)\right)^{2}}^{2}+\|\mathbf{u}\|_{\mathcal{B}\left(\varphi, Q_{\omega}\right)}^{2}\right), \quad \forall s \geq s_{0}(\tau, N), N>N_{0}, \operatorname{supp} \mathbf{u} \subset[0, T] \times \Omega_{1 / N^{2}},
\end{aligned}
$$

where the constant $C_{4}$ is independent of $s$ and $N$.

The proof of Lemma 4.1 is given in Sections $5-8$. Now, using the result of this lemma, we finish the proof of Theorem 2.1. Let us fix the parameter $N$ such that (4.5) holds true. We take $\widetilde{\delta} \in\left(0, \frac{1}{N^{2}}\right)$ sufficiently small such that

$$
\phi(x)>\varphi(x), \quad \forall x \in \overline{\Omega_{\widetilde{\delta}} \backslash \Omega_{\widetilde{\delta} / 2}} .
$$

We consider a cut off function $\widetilde{\theta} \in C^{3}\left(\bar{\Omega}_{\widetilde{\delta}}\right)$ such that $\left.\widetilde{\theta}\right|_{\Omega_{\frac{\delta}{2}}}=1$ and $\left.\widetilde{\theta}\right|_{\Omega_{\tilde{\delta}} \backslash \Omega_{\frac{3 \tilde{\delta}}{4}}}=0$. The function $\widetilde{\theta} \mathbf{u}$ satisfies the equation

$$
P(\widetilde{\theta} \mathbf{u})=\widetilde{\theta} \mathbf{f}+[P, \widetilde{\theta}] \mathbf{u},\left.\quad \mathbf{u}\right|_{(0, T) \times \partial \Omega}=0, \quad \mathbf{u}(0, \cdot)=\mathbf{u}_{x_{0}}(0, \cdot)=\mathbf{u}(T, \cdot)=\mathbf{u}_{x_{0}}(T, \cdot)=0 .
$$


Applying Carleman estimate (4.5) to this equation, we obtain

$$
\begin{aligned}
& s\left\|\frac{\partial \mathbf{u}}{\partial \vec{n}} \mathrm{e}^{s \phi}\right\|_{\left(H^{1}((0, T) \times \partial \Omega)\right)^{2}}^{2}+s\left\|\frac{\partial^{2} \mathbf{u}}{\partial \vec{n}^{2}} \mathrm{e}^{s \phi}\right\|_{\left(L^{2}((0, T) \times \partial \Omega)\right)^{2}}^{2}+s^{3}\left\|\frac{\partial \mathbf{u}}{\partial \vec{n}} \mathrm{e}^{s \phi}\right\|_{\left(L^{2}((0, T) \times \partial \Omega)\right)^{2}}^{2} \\
& \leq C_{8}\left(s^{2}\left\|\mathbf{f e}^{s \varphi}\right\|_{\left(L^{2}(Q)\right)^{2}}^{2}+\left\|(\nabla \mathbf{f}) \mathrm{e}^{s \varphi}\right\|_{\left(L^{2}(Q)\right)^{2}}^{2}+s^{2}\left\|[P, \widetilde{\theta}] \mathbf{u} \mathrm{e}^{s \varphi}\right\|_{\left(L^{2}(Q)\right)^{2}}^{2}\right. \\
& \left.\quad+\left\|\nabla([P, \widetilde{\theta}] \mathbf{u}) \mathrm{e}^{s \varphi}\right\|_{\left(L^{2}(Q)\right)^{2}}^{2}+\|\mathbf{u}\|_{\mathcal{B}\left(\phi, Q_{\omega}\right)}^{2}\right), \quad \forall s \geq s_{0}(\tau) .
\end{aligned}
$$

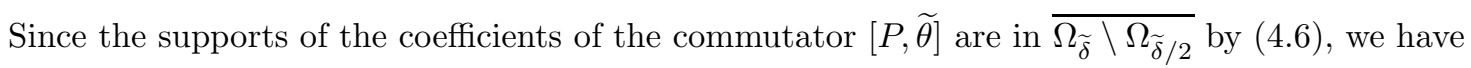

$$
\begin{aligned}
s^{2}\left\|[P, \widetilde{\theta}] \mathbf{u e}^{s \varphi}\right\|_{\left(L^{2}(Q)\right)^{2}}^{2}+\left\|\nabla([P, \widetilde{\theta}] \mathbf{u}) \mathrm{e}^{s \varphi}\right\|_{\left(L^{2}(Q)\right)^{2}}^{2} & =\|\mathbf{u}\|_{\mathcal{B}\left(\varphi, Q_{\omega}\right)}^{2} \\
& \leq C_{9}\left(\sum_{|\alpha|=0}^{2} s^{3-2|\alpha|}\left\|\left(\partial_{x}^{\alpha} \mathbf{u}\right) \mathrm{e}^{s \phi}\right\|_{\left(L^{2}(Q)\right)^{2}}^{2}+\|\mathbf{u}\|_{\mathcal{B}\left(\phi, Q_{\omega}\right)}^{2}\right) .
\end{aligned}
$$

Combining (4.7) and (4.8), we obtain

$$
\begin{aligned}
& s\left\|\frac{\partial \mathbf{u}}{\partial \vec{n}} \mathrm{e}^{s \phi}\right\|_{\left(H^{1}((0, T) \times \partial \Omega)\right)^{2}}^{2}+s\left\|\frac{\partial^{2} \mathbf{u}}{\partial \vec{n}^{2}} \mathrm{e}^{s \phi}\right\|_{\left(L^{2}((0, T) \times \partial \Omega)\right)^{2}}^{2}+s^{3}\left\|\frac{\partial \mathbf{u}}{\partial \vec{n}} \mathrm{e}^{s \phi}\right\|_{\left(L^{2}((0, T) \times \partial \Omega)\right)^{2}}^{2} \\
& \leq C_{10}\left(s^{2}\left\|\mathbf{f e}^{s \varphi}\right\|_{\left(L^{2}(Q)\right)^{2}}^{2}+\left\|(\nabla \mathbf{f}) \mathrm{e}^{s \varphi}\right\|_{\left(L^{2}(Q)\right)^{2}}^{2}+\sum_{|\alpha|=0}^{2} s^{3-2|\alpha|}\left\|\left(\partial_{x}^{\alpha} \mathbf{u}\right) \mathrm{e}^{s \phi}\right\|_{\left(L^{2}(Q)\right)^{2}}^{2}+\|\mathbf{u}\|_{\mathcal{B}\left(\phi, Q_{\omega}\right)}^{2}\right), \quad \forall s \geq s_{0}(\tau) .
\end{aligned}
$$

Finally we will estimate the surface integrals at the right hand side of (4.4) by the right hand side of (4.9). In the new inequality, the term

$$
\sum_{|\alpha|=0}^{2} s^{3-2|\alpha|}\left\|\left(\partial_{x}^{\alpha} \mathbf{u}\right) \mathrm{e}^{s \phi}\right\|_{\left(L^{2}(Q)\right)^{2}}^{2}
$$

which appears at the right hand side, can be absorbed by $\|\mathbf{u}\|_{Y(\phi, Q)}^{2}$. Thus the proof of Theorem 2.1 is complete.

\section{Proof of Lemma 4.1}

In this section, we will prove Lemma 4.1. Following the standard technique, we reduce the proof of estimate (4.5) to subelliptic estimate (5.13) for the operator $\mathbb{P}_{\sigma}$. Next show that we can act microlocally in this case. Namely we reduce estimate (5.13) to estimate (5.15). In the situation with the Lamé system this reduction is not trivial, since we have the subelliptic estimate with loss of one derivative. This difficulty is overcome with the help of the second large parameter $N$ inserted into the function $\varphi$. Finally we formulate several lemmata on factorization of pseudo-differential operators, a priori estimates of Cauchy problem for pseudo-differential operators, and Carleman estimate for a second order scalar hyperbolic equation, which are used in Sections 6-8.

Proof of Lemma 4.1. First we note that, thanks to the large parameter $N$, it suffices to prove (4.5) only locally by assuming

$$
\operatorname{supp} \mathbf{u} \subset B_{\delta} \cap\left([0, T] \times \Omega_{1 / N^{2}}\right)
$$

where $B_{\delta}$ is the ball of the radius $\delta>0$ centered at some point $y^{*}$. In the case of $B_{\delta} \cap((0, T) \times \partial \Omega)=\emptyset$, we can prove (4.5) in a usual way for a function with compact support (see e.g., [15]). Without loss of generality, we may assume that $y^{*}=\left(y_{0}^{*}, 0,0\right)$. Moreover the parameter $\delta>0$ can be chosen arbitrarily small. Assume that near $(0,0)$, the boundary $\partial \Omega$ is locally given by the equation $x_{2}-\ell\left(x_{1}\right)=0$. Furthermore, since the function 
$\widetilde{\mathbf{u}}=\mathcal{O} \mathbf{u}\left(x_{0}, \mathcal{O}^{-1} x^{\prime}\right)$ satisfies system $(2.1)$ and $(2.2)$ with $\widetilde{\mathbf{f}}=\mathcal{O} \mathbf{f}\left(x_{0}, \mathcal{O}^{-1} x^{\prime}\right)$ for any orthogonal matrix $\mathcal{O}$, we may assume that

$$
\ell^{\prime}(0) \equiv \frac{\mathrm{d} \ell}{\mathrm{d} x_{1}}(0)=0
$$

Making the change of variables $y_{1}=x_{1}$ and $y_{2}=x_{2}-\ell\left(x_{1}\right)$, we reduce equation (2.1) to the form

$$
\left\{\begin{array}{l}
\mathbb{P}_{1} \mathbf{u}=\frac{\partial^{2} u_{1}}{\partial y_{0}^{2}}-\mu\left(\frac{\partial^{2} u_{1}}{\partial y_{1}^{2}}-2 \ell^{\prime}\left(y_{1}\right) \frac{\partial^{2} u_{1}}{\partial y_{1} \partial y_{2}}+\left(1+\left|\ell^{\prime}\left(y_{1}\right)\right|^{2}\right) \frac{\partial^{2} u_{1}}{\partial y_{2}^{2}}\right)+\mu \ell^{\prime \prime}\left(y_{1}\right) \frac{\partial u_{1}}{\partial y_{2}} \\
-(\lambda+\mu) \frac{\partial}{\partial y_{1}}\left(\operatorname{div} \mathbf{u}-\frac{\partial u_{1}}{\partial y_{2}} \ell^{\prime}\right)+(\lambda+\mu) \frac{\partial}{\partial y_{2}}\left(\operatorname{div} \mathbf{u}-\frac{\partial u_{1}}{\partial y_{2}} \ell^{\prime}\right) \ell^{\prime}+\widetilde{K}_{1} \mathbf{u}=f_{1} \\
\mathbb{P}_{2} \mathbf{u}=\frac{\partial^{2} u_{2}}{\partial y_{0}^{2}}-\mu\left(\frac{\partial^{2} u_{2}}{\partial y_{1}^{2}}-2 \ell^{\prime}\left(y_{1}\right) \frac{\partial^{2} u_{2}}{\partial y_{1} \partial y_{2}}+\left(1+\left|\ell^{\prime}\left(y_{1}\right)\right|^{2}\right) \frac{\partial^{2} u_{2}}{\partial y_{2}^{2}}\right)+\mu \ell^{\prime \prime}\left(y_{1}\right) \frac{\partial u_{2}}{\partial y_{2}} \\
-(\lambda+\mu) \frac{\partial}{\partial y_{2}}\left(\operatorname{div} \mathbf{u}-\frac{\partial u_{1}}{\partial y_{2}} \ell^{\prime}\right)+\widetilde{K}_{2} \mathbf{u}=f_{2},
\end{array}\right.
$$

where we use the same notations $\mathbf{u}, \mathbf{f}$ after the change of variables and $\widetilde{K}_{1}, \widetilde{K}_{2}$ are partial differential operators of the first order. We set $\mathbb{P}=\left(\mathbb{P}_{1}, \mathbb{P}_{2}\right)$ and

$$
z_{1}=\frac{\partial u_{2}}{\partial y_{1}}-\frac{\partial u_{2}}{\partial y_{2}} \ell^{\prime}\left(y_{1}\right)-\frac{\partial u_{1}}{\partial y_{2}}, \quad z_{2}=\frac{\partial u_{1}}{\partial y_{1}}+\frac{\partial u_{2}}{\partial y_{2}}-\frac{\partial u_{1}}{\partial y_{2}} \ell^{\prime}\left(y_{1}\right)
$$

After the change of variables, equations (4.1) have the form

$$
\begin{gathered}
P_{\mu} z_{1}=\frac{\partial^{2} z_{1}}{\partial y_{0}^{2}}-\mu\left(\frac{\partial^{2} z_{1}}{\partial y_{1}^{2}}-2 \ell^{\prime}\left(y_{1}\right) \frac{\partial^{2} z_{1}}{\partial y_{1} \partial y_{2}}+\left(1+\left|\ell^{\prime}\left(y_{1}\right)\right|^{2}\right) \frac{\partial^{2} z_{1}}{\partial y_{2}^{2}}\right)+\mu \ell^{\prime \prime}\left(y_{1}\right) \frac{\partial z_{1}}{\partial y_{2}} \\
=m_{1} \quad \text { in } \mathcal{G}_{N} \triangleq \mathbb{R}^{2} \times\left[0, \frac{\widehat{\kappa}}{N^{2}}\right] \\
P_{\lambda+2 \mu} z_{2}=\frac{\partial^{2} z_{2}}{\partial y_{0}^{2}}-(\lambda+2 \mu)\left(\frac{\partial^{2} z_{2}}{\partial y_{1}^{2}}-2 \ell^{\prime}\left(y_{1}\right) \frac{\partial^{2} z_{2}}{\partial y_{1} \partial y_{2}}+\left(1+\left|\ell^{\prime}\left(y_{1}\right)\right|^{2}\right) \frac{\partial^{2} z_{2}}{\partial y_{2}^{2}}\right)+(\lambda+2 \mu) \ell^{\prime \prime}\left(y_{1}\right) \frac{\partial z_{2}}{\partial y_{2}} \\
=m_{2} \quad \text { in } \mathcal{G}_{N} .
\end{gathered}
$$

Here we use the same notations $m_{1}, m_{2}$ after the change of variables and the constant $\widehat{\kappa}>0$ is chosen sufficiently large such that the image of $\left([0, T] \times \Omega_{1 / N^{2}}\right) \cap B_{\delta}\left(y^{*}\right)$ belongs to $\mathcal{G}_{N}$. Henceforth we write $\left(z_{1}, z_{2}\right)=R(y, D) \mathbf{u}$, where

$$
D=\left(D_{y_{0}}, D_{y_{1}}, D_{y_{2}}\right), \quad D_{y_{j}}=\frac{1}{i} \partial_{y_{j}}, \quad j=0,1,2, \text { etc. }
$$

and $\bar{c}$ denotes the complex conjugate of $c \in \mathbb{C}$.

Now we claim that in order to prove Lemma 4.1, it suffices to establish the following estimate for the function $\mathbf{w}=\left(w_{1}, w_{2}\right)=\mathrm{e}^{s \varphi}\left(z_{1}, z_{2}\right)=\mathrm{e}^{s \varphi} R(y, D) \mathbf{u}:$

$$
\begin{aligned}
& \|\mathbf{w}\|_{*}^{2} \equiv s\|\mathbf{w}\|_{\left(H^{1}\left(\mathcal{G}_{N}\right)\right)^{2}}^{2}+s^{3}\|\mathbf{w}\|_{\left(L^{2}\left(\mathcal{G}_{N}\right)\right)^{2}}^{2}+s\left\|\frac{\partial \mathbf{w}}{\partial y_{2}}\right\|_{\left(L^{2}\left(\partial \mathcal{G}_{N}\right)\right)^{2}}^{2}+s\|\mathbf{w}\|_{\left(H^{1}\left(\partial \mathcal{G}_{N}\right)\right)^{2}}^{2} \\
& +s^{3}\|\mathbf{w}\|_{\left(L^{2}\left(\partial \mathcal{G}_{N}\right)\right)^{2}}^{2} \leq C_{5}\left(\left\|\mathbb{P} \mathbf{u e} e^{s \varphi}\right\|_{\left(H^{1}\left(\mathcal{G}_{N}\right)\right)^{2}}^{2}+s^{2}\left\|\mathbb{P} \mathbf{u e} e^{s \varphi}\right\|_{\left(L^{2}\left(\mathcal{G}_{N}\right)\right)^{2}}^{2}+s\|\mathbf{g}\|_{\left(L^{2}\left(\partial \mathcal{G}_{N}\right)\right)^{2}}^{2}\right. \\
& \left.+\sum_{|\alpha|=0}^{2} s^{4-2|\alpha|}\left\|\left(\partial_{y^{\prime}}^{\alpha} \mathbf{u}\right) \mathrm{e}^{s \varphi}\right\|_{\left(L^{2}\left(\mathcal{G}_{N}\right)\right)^{2}}^{2}\right), \quad \forall s \geq s_{0}(\tau, N),
\end{aligned}
$$


for all $\mathbf{u} \in\left(H^{2}\left(\mathcal{G}_{N}\right)\right)^{2}$ satisfying $\left.\mathbf{u}\right|_{\partial \mathcal{G}_{N}}=0$ and suppu $\subset B_{\delta} \cap \mathcal{G}_{N}$. Obviously the function $\mathbf{w}$ satisfies the boundary condition

$$
\begin{gathered}
\frac{\partial w_{1}}{\partial y_{2}}=\frac{\lambda+2 \mu}{\mu} \frac{\partial w_{2}}{\partial y_{1}}+s \varphi_{y_{2}}\left(y^{*}\right) w_{1}-s \frac{\lambda+2 \mu}{\mu} \varphi_{y_{1}}\left(y^{*}\right) w_{2}+g_{1}, \quad \text { on } \partial \mathcal{G}_{N}, \\
\frac{\partial w_{2}}{\partial y_{2}}=-\frac{\mu}{\lambda+2 \mu} \frac{\partial w_{1}}{\partial y_{1}}+s \varphi_{y_{2}}\left(y^{*}\right) w_{2}+s \frac{\mu}{\lambda+2 \mu} \varphi_{y_{1}}\left(y^{*}\right) w_{1}+g_{2}, \quad \text { on } \partial \mathcal{G}_{N},
\end{gathered}
$$

where the function $\mathbf{g}=\left(g_{1}, g_{2}\right)$ satisfies the estimate

$$
s\|\mathbf{g}\|_{\left(L^{2}\left(\partial \mathcal{G}_{N}\right)\right)^{2}}^{2} \leq \epsilon(\delta)\left(s\left\|\frac{\partial \mathbf{w}}{\partial y_{2}}\right\|_{\left(L^{2}\left(\partial \mathcal{G}_{N}\right)\right)^{2}}^{2}+s\|\mathbf{w}\|_{\left(H^{1}\left(\partial \mathcal{G}_{N}\right)\right)^{2}}^{2}+s^{3}\|\mathbf{w}\|_{\left(L^{2}\left(\partial \mathcal{G}_{N}\right)\right)^{2}}^{2}\right)+C_{6} s\left\|\mathbb{P} \mathbf{u} \mathbf{e}^{s \varphi}\right\|_{\left(L^{2}\left(\partial \mathcal{G}_{N}\right)\right)^{2}}^{2}
$$

and $\lim _{\delta \rightarrow 0} \epsilon(\delta)=0$.

Boundary Conditions (5.6) and (5.7) with property (5.8) follow from equation (5.2) and the zero Dirichlet boundary condition for $\mathbf{u}$.

In order to deduce (4.5) from estimate (5.5), it suffices to show

$$
\|\mathbf{u}\|_{Y\left(\varphi, \mathcal{G}_{N}\right)}^{2} \leq C_{7}\left(\|\mathbf{w}\|_{*}^{2}+\left\|\mathbb{P} \mathbf{u} \mathrm{e}^{s \varphi}\right\|_{\left(H^{1}\left(\mathcal{G}_{N}\right)\right)^{2}}^{2}+s^{2}\left\|\mathbb{P} \mathbf{u e}^{s \varphi}\right\|_{\left(L^{2}\left(\mathcal{G}_{N}\right)\right)^{2}}^{2}\right), \quad \forall s \geq s_{0}(\tau, N) .
$$

For the proof of (5.9), we need

Proposition 5.1. There exist $\widehat{\tau}>1$ and $N_{0}>1$ such that for any $\tau>\widehat{\tau}$ and $N>N_{0}(\tau)$, there exists $s_{0}(\tau, N)$ such that

$$
\begin{aligned}
& N \int_{\mathcal{G}_{N}}\left(\frac{1}{s \varphi} \sum_{j, k=1}^{2}\left|\partial_{y_{j}} \partial_{y_{k}} \mathbf{u}\right|^{2}+s \varphi\left|\partial_{y_{j}} \mathbf{u}\right|^{2}+s^{3} \varphi^{3}|\mathbf{u}|^{2}\right) \mathrm{e}^{2 s \varphi} \mathrm{d} y \\
& \leq C_{8}\left(\left\|z_{1} \mathrm{e}^{s \varphi}\right\|_{H^{1}\left(\mathcal{G}_{N}\right)}^{2}+\left\|z_{2} \mathrm{e}^{s \varphi}\right\|_{H^{1}\left(\mathcal{G}_{N}\right)}^{2}\right), \quad \forall \mathbf{u} \in\left(H_{0}^{1}\left(\mathcal{G}_{N}\right)\right)^{2}, \text { supp } \mathbf{u} \subset B_{\delta} \cap \mathcal{G}_{N}, \forall s \geq s_{0}(\tau, N),
\end{aligned}
$$

where the constant $C_{8}$ is independent of $N$.

We give the proof of Proposition 5.1 in Appendix I.

Thanks to Proposition 5.1 and equations (5.2), we obtain

$$
\begin{aligned}
& N\left\|\left(\partial_{y_{0}}^{2} \mathbf{u}\right) \mathrm{e}^{s \varphi}\right\|_{\left(L^{2}\left(\mathcal{G}_{N}\right)\right)^{2}}^{2}+\sum_{|\alpha|=0, \alpha=\left(0, \alpha_{1}, \alpha_{2}\right)}^{2} N s^{4-2|\alpha|}\left\|\left(\partial_{y^{\prime}}^{\alpha} \mathbf{u}\right) \mathrm{e}^{s \varphi}\right\|_{\left(L^{2}\left(\mathcal{G}_{N}\right)\right)^{2}}^{2} \\
& \leq C_{9}\left(\|w\|_{*}^{2}+N\left\|\mathbb{P} \mathbf{u e}^{s \varphi}\right\|_{\left.\left(L^{2}\left(\mathcal{G}_{N}\right)\right)^{2}\right)}^{2} \quad \forall s \geq s_{0}(\tau, N) .\right.
\end{aligned}
$$

By (5.5) and (5.8)-(5.10), we obtain

$$
\begin{aligned}
& N\left\|\left(\partial_{y_{0}}^{2} \mathbf{u}\right) \mathrm{e}^{s \varphi}\right\|_{\left(L^{2}\left(\mathcal{G}_{N}\right)\right)^{2}}^{2}+\sum_{|\alpha|=0, \alpha=\left(0, \alpha_{1}, \alpha_{2}\right)}^{2} N s^{4-2|\alpha|}\left\|\left(\partial_{y^{\prime}}^{\alpha} \mathbf{u}\right) \mathrm{e}^{s \varphi}\right\|_{\left(L^{2}\left(\mathcal{G}_{N}\right)\right)^{2}}^{2}+\|\mathbf{u}\|_{Y\left(\varphi, \mathcal{G}_{N}\right)}^{2} \\
& \leq C_{10}\left(\left\|\nabla(\mathbb{P} \mathbf{u}) \mathrm{e}^{s \varphi}\right\|_{\left(L^{2}\left(\mathcal{G}_{N}\right)\right)^{2}}^{2}+s^{2}\left\|\mathbb{P} \mathbf{u} e^{s \varphi}\right\|_{\left(L^{2}\left(\mathcal{G}_{N}\right)\right)^{2}}^{2}\right) \quad \forall s \geq \max \left\{s_{0}(\tau, N), N\right\} .
\end{aligned}
$$

Finally, combining (5.11) with the estimates

$$
s^{2}\left\|\left(\partial_{y_{0}} \mathbf{u}\right) \mathrm{e}^{s \varphi}\right\|_{\left(L^{2}\left(\mathcal{G}_{N}\right)\right)^{2}}^{2} \leq C_{11}\left(\left\|\left(\partial_{y_{0}}^{2} \mathbf{u}\right) \mathrm{e}^{s \varphi}\right\|_{\left(L^{2}\left(\mathcal{G}_{N}\right)\right)^{2}}^{2}+s^{4}\left\|\mathbf{u} \mathrm{e}^{s \varphi}\right\|_{\left(L^{2}\left(\mathcal{G}_{N}\right)\right)^{2}}^{2}\right)
$$


and

$$
\left\|\left(\partial_{y_{0}} \partial_{y_{k}} \mathbf{u}\right) \mathrm{e}^{s \varphi}\right\|_{\left(L^{2}\left(\mathcal{G}_{N}\right)\right)^{2}}^{2} \leq C_{11} \sum_{j=0}^{2}\left\|\left(\partial_{y_{j}}^{2} \mathbf{u}\right) \mathrm{e}^{s \varphi}\right\|_{\left(L^{2}\left(\mathcal{G}_{N}\right)\right)^{2}}^{2}, \quad k \in\{1,2\}
$$

we obtain (5.9).

Now we will proceed to the proof of (5.5). We set $P_{\mu, s}=\mathrm{e}^{|s| \varphi} P_{\mu} \mathrm{e}^{-|s| \varphi}$ and $P_{\lambda+2 \mu, s}=\mathrm{e}^{|s| \varphi} P_{\lambda+2 \mu} \mathrm{e}^{-|s| \varphi}$. By $\mathbf{p}\left(y, \xi_{0}, \xi_{1}, \xi_{2}\right)$ and $p_{\beta}\left(y, \xi_{0}, \xi_{1}, \xi_{2}\right)$ with $\beta=\mu$ or $\lambda+2 \mu$, we denote the principal symbols of the operators $\mathbb{P}$ and $P_{\beta}$ respectively. In order to prove Carleman estimate (5.5), it is convenient for us to introduce a new variable $\sigma$ and consider $s$ as a dual variable to $\sigma$. Following [46], Chapter 14, we consider the pseudo-differential operators defined by

$$
\begin{aligned}
& \mathbf{P}_{\beta}\left(y, D_{\sigma}, D_{y_{0}}, D_{y_{1}}, D_{y_{2}}\right) v=\int_{\mathbb{R}^{3}} p_{\beta}\left(y, \xi_{0}+i|s| \varphi_{y_{0}}, \xi_{1}+i|s| \varphi_{y_{1}}, D_{y_{2}}+i|s| \varphi_{y_{2}}\right) \widehat{v}\left(s, \xi_{0}, \xi_{1}, y_{2}\right) \mathrm{e}^{i\left(<y^{\prime}, \xi^{\prime}>+\sigma s\right)} \mathrm{d} \sigma \mathrm{d} \xi^{\prime} \\
& \mathbb{P}_{\sigma}\left(y, D_{\sigma}, D_{y_{0}}, D_{y_{1}}, D_{y_{2}}\right) v=\int_{\mathbb{R}^{3}} \mathbf{p}\left(y, \xi_{0}+i|s| \varphi_{y_{0}}, \xi_{1}+i|s| \varphi_{y_{1}}, D_{y_{2}}+i|s| \varphi_{y_{2}}\right) \widehat{v}\left(s, \xi_{0}, \xi_{1}, y_{2}\right) \mathrm{e}^{i\left(<y^{\prime}, \xi^{\prime}>+\sigma s\right)} \mathrm{d} \sigma \mathrm{d} \xi^{\prime}
\end{aligned}
$$

where $\xi^{\prime}=\left(\xi_{0}, \xi_{1}\right), y^{\prime}=\left(y_{0}, y_{1}\right)$ and $\widehat{v}\left(s, \xi_{0}, \xi_{1}, y_{2}\right)$ is the Fourier transform of $v\left(\sigma, y_{0}, y_{1}, y_{2}\right)$ with respect to $\sigma, y_{0}, y_{1}$. Let $\mathbf{v}(\sigma, y)=\left(v_{1}(\sigma, y), v_{2}(\sigma, y)\right)$ be a function with the domain $\mathcal{Q}=\mathbb{R}^{3} \times \mathbb{R}_{+}^{1}$. Henceforth $\mathcal{F}_{\sigma}$ denotes the Fourier transform with respect to the variable $\sigma$. Let $h(s)=\left(1+s^{2}\right)^{\frac{1}{4}}, \Sigma=\partial \mathcal{Q}$. Moreover we set $\mathbf{g}=\left(g_{1}, g_{2}\right)$,

$$
R_{s}(y, D) \mathcal{U}=\mathrm{e}^{|s| \varphi} R(y, D) \mathrm{e}^{-|s| \varphi} \mathcal{U}
$$

and

$$
\left\{\begin{array}{l}
B_{1} \mathbf{w} \triangleq-\frac{\partial w_{1}}{\partial y_{2}}+\frac{\lambda+2 \mu}{\mu} \frac{\partial w_{2}}{\partial y_{1}}+|s| \varphi_{y_{2}}\left(y^{*}\right) w_{1}-|s| \frac{\lambda+2 \mu}{\mu} \varphi_{y_{1}}\left(y^{*}\right) w_{2}, \\
B_{2} \mathbf{w} \triangleq-\frac{\partial w_{2}}{\partial y_{2}}-\frac{\mu}{\lambda+2 \mu} \frac{\partial w_{1}}{\partial y_{1}}+|s| \varphi_{y_{2}}\left(y^{*}\right) w_{2}+|s| \frac{\mu}{\lambda+2 \mu} \varphi_{y_{1}}\left(y^{*}\right) w_{1} \quad \text { on } \Sigma
\end{array}\right.
$$

for $\mathbf{w}=\left(w_{1}, w_{2}\right)$, provided that the right hand sides are well-defined.

Then we claim that in order to prove (5.5), it suffices to establish the following estimate

$$
\begin{aligned}
& \left.\quad\|\mathbf{v}\|\right|^{2} \triangleq \sum_{j=0}^{1}\left\|h\left(D_{\sigma}\right)^{3-2 j} \mathbf{v}\right\|_{L^{2}\left(\mathbb{R}^{1} ;\left(H^{j}\left(\mathcal{G}_{N}\right)\right)^{2}\right)}^{2}+\left\|h\left(D_{\sigma}\right)^{3-2 j} \mathbf{v}\right\|_{\left(H^{j}(\Sigma)\right)^{2}}^{2}+\left\|h\left(D_{\sigma}\right) \frac{\partial \mathbf{v}}{\partial y_{2}}\right\|_{\left(L^{2}(\Sigma)\right)^{2}}^{2} \\
& \leq C_{12}\left(\left\|\mathbb{P}_{\sigma}(y, D) \mathcal{F}_{\sigma}^{-1} \mathcal{U}\right\|_{\left(H^{1}(\mathcal{Q})\right)^{2}}^{2}+\left\|h\left(D_{\sigma}\right) \mathcal{F}_{\sigma}^{-1} \mathbf{g}\right\|_{\left(L^{2}(\Sigma)\right)^{2}}^{2}+\left\|\mathcal{F}_{\sigma}^{-1} \mathcal{U}\right\|_{\left(H^{2}(\mathcal{Q})\right)^{2}}^{2}\right),
\end{aligned}
$$

if $\mathcal{U}$ and $\mathbf{v}$ satisfy $\operatorname{supp} \mathcal{U} \subset \mathbb{R}^{1} \times\left(B_{\delta} \cap \mathcal{G}_{N}\right)$, supp $\mathcal{F}_{\sigma}^{-1} \mathcal{U} \subset\left(-\sigma_{0}, \sigma_{0}\right) \times\left(B_{\delta} \cap \mathcal{G}_{N}\right)$ with arbitrarily small parameter $\sigma_{0}>0$, and

$$
\left\{\begin{array}{l}
R_{s}(y, D) \mathcal{U}=\mathcal{F}_{\sigma} \mathbf{v},\left.\quad \mathcal{U}\right|_{\Sigma}=0 \\
B_{1}\left(\mathcal{F}_{\sigma} \mathbf{v}\right)=g_{1}, \quad B_{2}\left(\mathcal{F}_{\sigma} \mathbf{v}\right)=g_{2} \quad \text { on } \Sigma
\end{array}\right.
$$

We set

$$
\mathcal{F}_{\sigma} \mathbf{v}=\mathbf{w}
$$

Then

$$
\left(B_{1} \mathbf{w}, B_{2} \mathbf{w}\right)=\left(g_{1}, g_{2}\right) \equiv \mathbf{g} .
$$

This fact can be proved exactly in the same way as in [46], Chapter 14, Section 2.

Consider the finite covering of the unit sphere $\mathbb{S}^{2} \equiv\left\{\left(s, \xi_{0}, \xi_{1}\right) ; s^{2}+\xi_{0}^{2}+\xi_{1}^{2}=1\right\}: \mathbb{S}^{2} \subset \cup_{\zeta^{*} \in S^{2}}\left\{\zeta=\left(s, \xi_{0}, \xi_{1}\right) \in\right.$ $\left.\mathbb{S}^{2} ;\left|\zeta-\zeta^{*}\right|<\delta_{1}\right\}$ and the partition of unity $\chi_{\nu}(\zeta): \sum_{\nu=1}^{K\left(\delta_{1}\right)} \chi_{\nu}(\zeta)=1$ for any $\zeta \in \mathbb{S}^{2}$ and $\operatorname{supp} \chi_{\nu} \subset\{\zeta \in$ $\left.\mathbb{S}^{2} ;\left|\zeta-\zeta_{\nu}^{*}\right|<\delta_{1}\right\}$. 
We extend the function $\chi_{\nu}$ on the set $|\zeta|>1$ as the homogeneous function of the order zero in such a way that

$$
\operatorname{supp} \chi_{\nu} \subset \mathcal{O}\left(\delta_{1}\right) \equiv\left\{\zeta ;\left|\frac{\zeta}{|\zeta|}-\zeta^{*}\right|<\delta_{1}\right\},
$$

and continue $\chi_{\nu}$ on the set $|\zeta|<1$ up to a $C^{\infty}$ function.

We set $D^{\prime}=\left(D_{\sigma}, D_{y_{0}}, D_{y_{1}}\right)$, and consider the pseudo-differential operator $\chi_{\nu}\left(D^{\prime}\right)$ and the function $\chi_{\nu}\left(D^{\prime}\right) \mathbf{v}$. Obviously equalities (5.14) hold true with $\mathbf{w}$ and $\mathbf{g}$ replaced by $\mathbf{w}_{\nu}=\frac{1}{\sqrt{2 \pi}} \int_{-\infty}^{+\infty} \chi_{\nu}\left(D^{\prime}\right) \mathbf{v e} \mathrm{e}^{-i s \sigma} \mathrm{d} \sigma$ and $\mathbf{g}_{\nu}=$ $\frac{1}{\sqrt{2 \pi}} \int_{-\infty}^{+\infty} \chi_{\nu}\left(D^{\prime}\right) \mathcal{F}_{\sigma}^{-1} \mathbf{g e}^{-i s \sigma} \mathrm{d} \sigma$

Moreover we claim that instead of (5.13), it suffices to prove the following estimate

$$
\left\|\chi_{\nu}\left(D^{\prime}\right) \mathbf{v}\right\| \mid \leq C_{13}\left(\left\|\mathbb{P}_{\sigma} \chi_{\nu}\left(D^{\prime}\right) \mathcal{F}_{\sigma}^{-1} \mathcal{U}\right\|_{\left(H^{1}(\mathcal{Q})\right)^{2}}+\left\|h\left(D_{\sigma}\right) \chi_{\nu}\left(D^{\prime}\right) \mathcal{F}_{\sigma}^{-1} \mathbf{g}\right\|_{\left(L^{2}(\Sigma)\right)^{2}}+\left\|\mathcal{F}_{\sigma}^{-1} \mathcal{U}\right\|_{\left(H^{2}(\mathcal{Q})\right)^{2}}\right)
$$

where

$R_{s}\left(y, D^{\prime}\right) \mathcal{U}=\mathcal{F}_{\sigma} \mathbf{v},\left.\quad \mathcal{U}\right|_{\Sigma}=0, \quad \operatorname{supp} \mathcal{F}_{\sigma}^{-1} \mathcal{U} \subset\left(-\sigma_{0}, \sigma_{0}\right) \times\left(B_{\delta} \cap \mathcal{G}_{N}\right)$,

$$
B_{1}\left(w_{1, \nu}, w_{2, \nu}\right)=g_{1, \nu}, \quad B_{2}\left(w_{1, \nu}, w_{2, \nu}\right)=g_{2, \nu}
$$

and $C_{13}$ is independent of $N$. In fact, assume that estimate (5.15) is already proved. Then

$$
\begin{aligned}
& \|\mid \mathbf{v}\|\left\|^{2} \leq \sum_{\nu=1}^{K\left(\delta_{1}\right)}\right\| \chi_{\nu}\left(D^{\prime}\right) \mathbf{v} \|^{2} \\
& \leq C_{14} \sum_{\nu=1}^{K}\left(\left\|\mathbb{P}_{\sigma}(y, D) \chi_{\nu} \mathcal{F}_{\sigma}^{-1} \mathcal{U}\right\|_{\left(H^{1}(\mathcal{Q})\right)^{2}}^{2}+\left\|h(s) \mathbf{g}_{\nu}\right\|_{\left(L^{2}(\Sigma)\right)^{2}}^{2}+\left\|\chi_{\nu}\left(D^{\prime}\right) \mathcal{F}_{\sigma}^{-1} \mathcal{U}\right\|_{\left(H^{2}(\mathcal{Q})\right)^{2}}^{2}\right) \\
& \leq C_{15} \sum_{\nu=1}^{K}\left(\left\|\chi_{\nu}\left(D^{\prime}\right) \mathbb{P}_{\sigma}(y, D) \mathcal{F}_{\sigma}^{-1} \mathcal{U}\right\|_{\left(H^{1}(\mathcal{Q})\right)^{2}}^{2}+\left\|\left[\chi_{\nu}\left(D^{\prime}\right), \mathbb{P}_{\sigma}\left(y, D^{\prime}\right)\right] \mathcal{F}_{\sigma}^{-1} \mathcal{U}\right\|_{\left(H^{1}(\mathcal{Q})\right)^{2}}^{2}\right. \\
& \left.\quad+\left\|h(s) \mathbf{g}_{\nu}\right\|_{\left(L^{2}(\Sigma)\right)^{2}}^{2}+\left\|\chi_{\nu}\left(D^{\prime}\right) \mathcal{F}_{\sigma}^{-1} \mathcal{U}\right\|_{\left(H^{1}(\mathcal{Q})\right)^{2}}^{2}\right) \\
& \leq C_{16}\left(\left\|\mathbb{P}_{\sigma}(y, D) \mathcal{F}_{\sigma}^{-1} \mathcal{U}\right\|_{\left(H^{1}(\mathcal{Q})\right)^{2}}^{2}+\|h(s) \mathbf{g}\|_{\left(L^{2}(\Sigma)\right)^{2}}^{2}+\left\|\mathcal{F}_{\sigma}^{-1} \mathcal{U}\right\|_{\left(H^{2}(\mathcal{Q})\right)^{2}}^{2}\right)
\end{aligned}
$$

where $K=K\left(\delta_{1}\right)$ and $C_{16}$ are independent of $N$.

Estimate (5.15) follows from Lemmas 6.1, 7.1 and 8.1 which are proved in Sections 6-8.

Now we formulate some results and introduce some definitions which will be used in the proof of estimate (5.15).

The principal symbol of the operator $P_{\beta, s}$ has the form

$$
p_{\beta}\left(y, s, \xi_{0}, \xi_{1}\right)=-\left(\xi_{0}+i|s| \varphi_{y_{0}}\right)^{2}+\beta\left[\left(\xi_{1}+i|s| \varphi_{y_{1}}\right)^{2}-2 \ell^{\prime}\left(\xi_{1}+i|s| \varphi_{y_{1}}\right)\left(\xi_{2}+i|s| \varphi_{y_{2}}\right)+\left(\xi_{2}+i|s| \varphi_{y_{2}}\right)^{2}|G|^{2}\right]
$$

where $|G|^{2}=1+\left(\ell^{\prime}\left(y_{1}\right)\right)^{2}$. The roots of this polynomial with respect to the variable $\xi_{2}$, are

$$
\begin{gathered}
\Gamma_{\beta}^{ \pm}\left(y, s, \xi_{0}, \xi_{1}\right)=-i|s| \varphi_{y_{2}}(y)+\alpha_{\beta}^{ \pm}\left(y, s, \xi_{0}, \xi_{1}\right), \\
\alpha_{\beta}^{ \pm}\left(y, s, \xi_{0}, \xi_{1}\right)=\frac{\left(\xi_{1}+i|s| \varphi_{y_{1}}(y)\right) \ell^{\prime}\left(y_{1}\right)}{|G|^{2}} \pm \sqrt{r_{\beta}\left(y, s, \xi_{0}, \xi_{1}\right)}, \\
r_{\beta}(y, \zeta)=\frac{\left(\left(\xi_{0}+i|s| \varphi_{y_{0}}(y)\right)^{2}-\beta\left(\xi_{1}+i|s| \varphi_{y_{1}}(y)\right)^{2}\right)|G|^{2}+\beta\left(\xi_{1}+i|s| \varphi_{y_{1}}\right)^{2}\left(\ell^{\prime}\right)^{2}}{\beta|G|^{4}},
\end{gathered}
$$

where the function $\sqrt{r_{\beta}}$ is defined below. 
Denote $\gamma=\left(y^{*}, \zeta^{*}\right)=\left(y^{*}, s^{*}, \xi_{0}^{*}, \xi_{1}^{*}\right)$.

Proposition 5.2. Suppose that $\left|r_{\beta}(\gamma)\right| \geq 2 \widehat{\delta}>0$. Then there exists $\delta_{0}=\delta_{0}(\widehat{\delta})>0$ such that for all $\delta, \delta_{1} \in\left(0, \delta_{0}\right)$, there exists a constant $C_{20}>0$, independent of $s$, such that for one of the roots of polynomial (5.17), which we denote by $\Gamma_{\beta}^{-}$, we have

$$
-\operatorname{Im} \Gamma_{\beta}^{-}\left(y, s, \xi_{0}, \xi_{1}\right) \geq C_{20}|s|, \quad \forall\left(y, s, \xi_{0}, \xi_{1}\right) \in B_{\delta} \times \mathcal{O}\left(\delta_{1}\right)
$$

Proof of Proposition 5.2. If $\operatorname{Im} \sqrt{r_{\beta}(\gamma)} \neq 0$, then statement (5.21) is trivial. So it suffices to consider the case $\operatorname{Im} \sqrt{r_{\beta}(\gamma)}=0$. Let $\theta \in\left(0, \frac{1}{8}\right)$ be a constant. Obviously there exists $\widetilde{\delta}(\theta)$ such that for all $\delta, \delta_{1} \in(0, \widetilde{\delta}(\theta))$,

$$
\operatorname{Re} r_{\beta}(y, \zeta) \geq(1-2 \theta)\left|r_{\beta}(y, \zeta)\right|, \quad \forall\left(y, s, \xi_{0}, \xi_{1}\right) \in B_{\delta} \times \mathcal{O}\left(\delta_{1}\right) .
$$

Then

$$
\left|\operatorname{Im} r_{\beta}(y, \zeta)\right| \leq \frac{2 \theta}{1-2 \theta} \operatorname{Re} r_{\beta}(y, \zeta), \quad \forall\left(y, s, \xi_{0}, \xi_{1}\right) \in B_{\delta} \times \mathcal{O}\left(\delta_{1}\right)
$$

We denote $b(y, \zeta)=\operatorname{Im} r_{\beta}(y, \zeta)$ and $a(y, \zeta)=\operatorname{Re} r_{\beta}(y, \zeta)$ with $\zeta=\left(s, \xi_{0}, \xi_{1}\right)$. First, if $\operatorname{Im} \sqrt{r_{\beta}(\gamma)}=0$, then we have $a(\gamma)>0$ and $b(\gamma)=0$. In that case we define the function $\sqrt{r_{\beta}(y, \zeta)}$ by the infinite series

$$
(1+x)^{\frac{1}{2}}=\sum_{n=0}^{\infty} c_{n} x^{n}, \quad|x|<1
$$

where $c_{n}=\frac{\frac{1}{2}\left(\frac{1}{2}-1\right)\left(\frac{1}{2}-2\right) \ldots\left(\frac{1}{2}-(n-1)\right)}{n !}$.

That is, assuming that $\left|\frac{b}{a}\right|<\frac{2 \theta}{1-2 \theta}<\frac{1}{2}$ for all $\left(y, s, \xi_{0}, \xi_{1}\right) \in B_{\delta} \times \mathcal{O}\left(\delta_{1}\right)$, we set

$$
\sqrt{r_{\beta}(y, \zeta)}=\sqrt{a} \sum_{n=0}^{\infty} c_{n}\left(\frac{i b}{a}\right)^{n}=\sqrt{a}+\frac{i}{2}|s|\left(\frac{b}{|s| \sqrt{a}}\right)-|s|\left(\frac{b}{a}\right) \frac{b}{\mid s \sqrt{a}} \sum_{n=0}^{\infty} c_{n+2}\left(\frac{i b}{a}\right)^{n} .
$$

The first term in infinite series (5.22) is real, and the absolute value of the third term is ||$s\left|\frac{b}{|s| \sqrt{a}}\right| O(\theta)$. The function $\frac{b}{|s| \sqrt{a}}$ is a continuous homogeneous function of the order zero in the variable $\zeta$.

If $\frac{b(\gamma)}{\mid s^{*} \sqrt{a(\gamma)}} \leq 0$, then we take $\Gamma_{\beta}^{-}(y, \zeta)=-i|s| \frac{\partial \varphi}{\partial y_{2}}+\alpha_{\beta}^{-}(y, \zeta)$ where $\alpha_{\beta}^{-}(y, \zeta)$ equals the right hand side of (5.22) plus $\left(\xi_{1}+i|s| \varphi_{y_{1}}\right) \ell^{\prime}\left(y_{1}\right) /|G|^{2}$. Otherwise $\Gamma_{\beta}^{-}(y, \zeta)=-i|s| \frac{\partial \varphi}{\partial y_{2}}+\alpha_{\beta}^{+}(y, \zeta)$ where $\alpha_{\beta}^{+}(y, \zeta)$ equals the right hand side of $(5.22)$ multiplied by -1 plus $\left(\xi_{1}+i|s| \varphi_{y_{1}}\right) \ell^{\prime}\left(y_{1}\right) /|G|^{2}$.

For $\frac{b}{\mid s^{*} \sqrt{a}}(\gamma) \leq 0$, we obtain that $\frac{b}{|s| \sqrt{a}}(\gamma)-\frac{1}{2} \varphi_{y_{2}}(y)<0$ for all $\left(y, s, \xi_{0}, \xi_{1}\right) \in B_{\delta} \times \mathcal{O}\left(\delta_{1}\right)$ and for $\frac{b}{\mid s^{*} \sqrt{a}}(\gamma) \geq 0$ we obtain that $-\frac{b}{|s| \sqrt{a}}(\gamma)-\frac{1}{2} \varphi_{y_{2}}(y)<0$ for all $\left(y, s, \xi_{0}, \xi_{1}\right) \in B_{\delta} \times \mathcal{O}\left(\delta_{1}\right)$. These inequalities imply $(5.21)$ provided that $\delta_{1}$ is taken sufficiently small. The proof of Proposition 5.2 is finished.

Under some conditions, we can see that the operator $\mathbf{P}_{\beta}$ can be factorized as a product of two first order pseudo-differential operators:

Proposition 5.3. Let $\beta \in\{\mu, \lambda+2 \mu\}$ and $\left|r_{\beta}(y, \zeta)\right| \geq \widehat{\delta}>0$ for all $(y, \zeta) \in B_{\delta} \times \mathcal{O}\left(2 \delta_{1}\right)$. Then we can factorize the operator $\mathbf{P}_{\beta}$ as the product of two first order pseudo-differential operators:

$$
\mathbf{P}_{\beta} \chi_{\nu}\left(D^{\prime}\right) V=\beta|G|^{2}\left(D_{y_{2}}-\Gamma_{\beta}^{-}\left(y, D^{\prime}\right)\right)\left(D_{y_{2}}-\Gamma_{\beta}^{+}\left(y, D^{\prime}\right)\right) \chi_{\nu}\left(D^{\prime}\right) V+T_{\beta} V,
$$

where $\operatorname{supp} V \subset B_{\delta} \cap \mathcal{G}_{N}$ and $T_{\beta}$ is a continuous operator:

$$
T_{\beta}: L^{2}\left(0,1 ; H^{1}\left(\mathbb{R}^{3}\right)\right) \rightarrow L^{2}\left(0,1 ; L^{2}\left(\mathbb{R}^{3}\right)\right) .
$$


Let us consider the equation

$$
\left(D_{y_{2}}-\Gamma_{\beta}^{-}\left(y, D^{\prime}\right)\right) \chi_{\nu}\left(D^{\prime}\right) V=q,\left.\quad V\right|_{y_{2}=\frac{\hat{\kappa}}{N^{2}}}=0, \quad \operatorname{supp} V \subset B_{\delta} \cap \mathcal{G}_{N} .
$$

For the solutions to this problem, we have an a priori estimate:

Proposition 5.4. Let $\beta \in\{\mu, \lambda+2 \mu\}$ and $\left|r_{\beta}(y, \zeta)\right| \geq \widehat{\delta}>0$ for all $(y, \zeta) \in B_{\delta} \times \mathcal{O}\left(2 \delta_{1}\right)$. Then there exists a constant $C_{22}>0$, which is independent of $N$, such that

$$
\left\|\left.h\left(D_{\sigma}\right) \chi_{\nu}\left(D^{\prime}\right) V\right|_{y_{2}=0}\right\|_{L^{2}\left(\mathbb{R}^{3}\right)} \leq C_{22}\|q\|_{L^{2}(\mathcal{Q})} .
$$

Proof of Proposition 5.4. Taking the scalar product of $q$ and $h^{2}\left(D_{\sigma}\right) \chi_{\nu}\left(D^{\prime}\right) V$ for fixed $y_{2}$, we obtain

$$
\begin{aligned}
2 \operatorname{Re}\left(q\left(y_{2}\right), h^{2}\left(D_{\sigma}\right) \chi_{\nu}\left(D^{\prime}\right) V\left(y_{2}\right)\right)_{L^{2}(\Sigma)} \mathrm{e}^{2 \widetilde{\kappa} y_{2}} & =\frac{\partial}{\partial y_{2}}\left(\mathrm{e}^{2 \widetilde{\kappa} y_{2}}\left\|h\left(D_{\sigma}\right) \chi_{\nu}\left(D^{\prime}\right) V\left(y_{2}\right)\right\|_{L^{2}(\Sigma)}^{2}\right) \\
& -2 \operatorname{Re}\left(i \Gamma_{\beta}^{-}\left(y, D^{\prime}\right) \chi_{\nu}\left(D^{\prime}\right) V+\widetilde{\kappa} \chi_{\nu}\left(D^{\prime}\right) V, h^{2}\left(D_{\sigma}\right) \chi_{\nu}\left(D^{\prime}\right) V\right)_{L^{2}(\Sigma)} \mathrm{e}^{2 \widetilde{\kappa} y_{2}} .
\end{aligned}
$$

By (5.21) and Proposition 2.4.A in [47], for sufficiently large positive $\widetilde{\kappa}$, we have

$$
\operatorname{Re}\left(i \Gamma_{\beta}^{-}\left(y, D^{\prime}\right) h^{-2}\left(D_{\sigma}\right) h^{2}\left(D_{\sigma}\right) \chi_{\nu}\left(D^{\prime}\right) V+\widetilde{\kappa} \chi_{\nu}\left(D^{\prime}\right) V, h^{2}\left(D_{\sigma}\right) \chi_{\nu}\left(D^{\prime}\right) V\right)_{L^{2}(\Sigma)} \geq C_{23}\left\|h^{2}\left(D_{\sigma}\right) \chi_{\nu}\left(D^{\prime}\right) V\right\|_{L^{2}(\Sigma)}^{2} .
$$

Thus

$$
\begin{aligned}
& 2 \operatorname{Re}\left(q\left(y_{2}\right), h^{2}\left(D_{\sigma}\right) \chi_{\nu}\left(D^{\prime}\right) V\left(y_{2}\right)\right)_{L^{2}(\Sigma)} \mathrm{e}^{2 \widetilde{\kappa} y_{2}} \\
& \quad \leq \frac{\partial}{\partial y_{2}}\left(\mathrm{e}^{2 \widetilde{\kappa} y_{2}}\left\|h\left(D_{\sigma}\right) \chi_{\nu}\left(D^{\prime}\right) V\left(y_{2}\right)\right\|_{L^{2}(\Sigma)}^{2}\right)-C_{23}\left\|h^{2}\left(D_{\sigma}\right) \chi_{\nu}\left(D^{\prime}\right) V\left(y_{2}\right)\right\|_{L^{2}(\Sigma)}^{2} \mathrm{e}^{2 \widetilde{\kappa} y_{2}},
\end{aligned}
$$

and (5.24) follows from Gronwall's inequality.

Let $\widetilde{w}(s, y)$ satisfy a scalar second order hyperbolic equation

$$
P_{\beta, s} \widetilde{w}=q \quad \text { in } \mathcal{G}_{N},\left.\quad \frac{\partial \widetilde{w}}{\partial y_{2}}\right|_{y_{2}=1}=\left.\widetilde{w}\right|_{y_{2}=1}=0, \quad \operatorname{supp} \widetilde{w} \subset \mathbb{R}^{1} \times\left(B_{\delta} \cap \mathcal{G}_{N}\right)
$$

for almost all $s \in \mathbb{R}^{1}$. Let $P_{\beta, s}^{*}$ be the formally adjoint operator to $P_{\beta, s}$, where $\beta \in\{\mu, \lambda+2 \mu\}$. Set

$$
L_{+, \beta}=\frac{P_{\beta, s}+P_{\beta, s}^{*}}{2}, \quad L_{-, \beta}=\frac{P_{\beta, s}-P_{\beta, s}^{*}}{2} .
$$

One can easily check that the principal part operator $L_{-, \beta}$ is given by formula

$$
L_{-, \beta} \widetilde{w}=-2|s| \varphi_{y_{0}} \frac{\partial \widetilde{w}}{\partial y_{0}}+\beta\left(2|s| \varphi_{y_{1}} \frac{\partial \widetilde{w}}{\partial y_{1}}-2|s| \ell^{\prime}\left(y_{1}\right)\left(\varphi_{y_{2}} \frac{\partial \widetilde{w}}{\partial y_{1}}+\varphi_{y_{1}} \frac{\partial \widetilde{w}}{\partial y_{2}}\right)+2|s|\left(1+\left(\ell^{\prime}\left(y_{1}\right)\right)^{2}\right) \varphi_{y_{2}} \frac{\partial \widetilde{w}}{\partial y_{2}}\right) .
$$

Obviously $L_{+, \beta} \widetilde{w}+L_{-, \beta} \widetilde{w}=q$. For almost all $s \in \mathbb{R}^{1}$, the following equality holds true:

$$
B_{\beta}+\left\|L_{-, \beta} \widetilde{w}\right\|_{L^{2}\left(\mathcal{G}_{N}\right)}^{2}+\left\|L_{+, \beta} \widetilde{w}\right\|_{L^{2}\left(\mathcal{G}_{N}\right)}^{2}+\operatorname{Re} \int_{\mathcal{G}_{N}}\left(\left[L_{+, \beta}, L_{-, \beta}\right] \widetilde{w}, \overline{\widetilde{w}}\right) \mathrm{d} y=\|q\|_{L^{2}\left(\mathcal{G}_{N}\right)}^{2},
$$


where

$B_{\beta}=\operatorname{Re} \int_{\partial \mathcal{G}_{N}} \widetilde{p}_{\beta}\left(y, \nabla \varphi,-\vec{e}_{3}\right)\left(|s| \widetilde{p}_{\beta}(y, \nabla \widetilde{w})-|s|^{3} \widetilde{p}_{\beta}(y, \nabla \varphi, \nabla \varphi) \widetilde{w}^{2}\right) \mathrm{d} y_{0} \mathrm{~d} y_{1}+\operatorname{Re} \int_{\partial \mathcal{G}_{N}} \widetilde{p}_{\beta}\left(y, \nabla \widetilde{w},-\vec{e}_{3}\right) \overline{L_{-, \beta} \widetilde{w}} \mathrm{~d} y_{0} \mathrm{~d} y_{1}$,

$\vec{e}_{3}=(0,0,1)$ and

$$
\widetilde{p}_{\beta}(y, \xi, \widetilde{\xi})=\xi_{0} \widetilde{\xi}_{0}-\beta\left(\xi_{1} \widetilde{\xi}_{1}-\ell^{\prime}\left(y_{1}\right)\left(\xi_{1} \widetilde{\xi}_{2}+\xi_{2} \widetilde{\xi}_{1}\right)+\left(1+\left|\ell^{\prime}\left(y_{1}\right)\right|^{2}\right) \xi_{2} \widetilde{\xi}_{2}\right)
$$

We note that $\left.\phi_{y_{k}}\right|_{\Sigma}=\left.\varphi_{y_{k}}\right|_{\Sigma}$ for $k \in\{0,1\}$ and $\left.\varphi_{y_{2}}\right|_{\Sigma}=\left.\left(\phi_{y_{2}}-\widehat{\epsilon} \tau\left(\partial_{y_{2}} \ell_{1}\right) \phi\right)\right|_{\Sigma}$. Therefore on $\Sigma$ the function $\nabla \varphi$ is independent of $N$ and $|\nabla \phi(y)-\nabla \varphi(y)| \leq C_{25} \widehat{\epsilon}$ for all $y \in \Sigma$ where $C_{25}>0$ is independent of $\widehat{\epsilon}$ and $N$. In particular, taking $\widehat{\epsilon}$ sufficiently small, we have (2.6) for the function $\varphi$. It is convenient for us to rewrite (5.26) in the form

$$
\begin{gathered}
B_{\beta}=B_{\beta}^{(1)}+B_{\beta}^{(2)} \\
B_{\beta}^{(1)} \equiv \operatorname{Re} \int_{y_{2}=0} 2|s| \beta \frac{\partial \widetilde{w}}{\partial y_{2}} \overline{\left(\beta \frac{\partial \widetilde{w}}{\partial y_{1}} \varphi_{y_{1}}\left(y^{*}\right)+\beta \frac{\partial \widetilde{w}}{\partial y_{2}} \varphi_{y_{2}}\left(y^{*}\right)-\frac{\partial \widetilde{w}}{\partial y_{0}} \varphi_{y_{0}}\left(y^{*}\right)\right)} \mathrm{d} y_{0} \mathrm{~d} y_{1} \\
+\int_{y_{2}=0}|s| \beta \varphi_{y_{2}}\left(y^{*}\right)\left\{\left|\frac{\partial \widetilde{w}}{\partial y_{0}}\right|^{2}-\beta\left(\left|\frac{\partial \widetilde{w}}{\partial y_{1}}\right|^{2}+\left|\frac{\partial \widetilde{w}}{\partial y_{2}}\right|^{2}\right)\right. \\
\left.-|s|^{2}\left(\varphi_{y_{0}}^{2}\left(y^{*}\right)-\beta\left(\varphi_{y_{1}}^{2}\left(y^{*}\right)+\varphi_{y_{2}}^{2}\left(y^{*}\right)\right)\right)|\widetilde{w}|^{2}\right\} \mathrm{d} y_{0} \mathrm{~d} y_{1} .
\end{gathered}
$$

Then

$$
\left|B_{\beta}^{(2)}\right| \leq \epsilon_{0}\left(|s|\left\|\frac{\partial \widetilde{w}}{\partial y_{2}}\right\|_{L^{2}\left(\partial \mathcal{G}_{N}\right)}^{2}+|s|\|\widetilde{w}\|_{H^{1}\left(\partial \mathcal{G}_{N}\right)}^{2}+|s|^{3}\|\widetilde{w}\|_{L^{2}\left(\partial \mathcal{G}_{N}\right)}^{2}\right),
$$

where $\epsilon_{0}=\epsilon_{0}(\delta) \rightarrow 0$ as $|\delta| \rightarrow 0$. It is known (see e.g., [18]) that there exists a parameter $\widehat{\tau}>1$ such that for any $\tau>\widehat{\tau}$, there exists $s_{0}(\tau)$ such that

$$
\begin{aligned}
\left\|L_{-, \beta} \widetilde{w}\right\|_{L^{2}\left(\mathcal{G}_{N}\right)}^{2} & +\left\|L_{+, \beta} \widetilde{w}\right\|_{L^{2}\left(\mathcal{G}_{N}\right)}^{2}+\operatorname{Re} \int_{\mathcal{G}_{N}}\left(\left[L_{+, \beta}, L_{-, \beta}\right] \widetilde{w}, \widetilde{w}\right) \mathrm{d} y \\
& +C_{26}^{\prime}|s|\|\widetilde{w}\|_{L^{2}\left(\partial \mathcal{G}_{N}\right)}\left\|\partial_{y_{2}} \widetilde{w}\right\|_{L^{2}\left(\partial \mathcal{G}_{N}\right)} \geq C_{26}\left(|s|\|\widetilde{w}\|_{H^{1}\left(\mathcal{G}_{N}\right)}^{2}+|s|^{3}\|\widetilde{w}\|_{L^{2}\left(\mathcal{G}_{N}\right)}^{2}\right), \quad \forall|s| \geq s_{0}(\tau),
\end{aligned}
$$

where $C_{26}>0$ is independent of $s$. We also claim that the constant $C_{26}$ is independent of $N$. The proof of estimate (5.28) is given in Appendix II.

Set

$$
\Xi_{\beta}=\int_{-\infty}^{\infty} B_{\beta} \mathrm{d} s, \quad \Xi_{\beta}^{(j)}=\int_{-\infty}^{\infty} B_{\beta}^{(j)} \mathrm{d} s, \quad j=1,2 .
$$

Therefore, integrating $(5.28)$ with respect to $s$ in $\mathbb{R}^{1}$, we have

$$
\begin{aligned}
& C_{27}\left(\|h(s) \widetilde{w}\|_{H^{1}(\mathcal{Q})}^{2}+\left\|h^{3}(s) \widetilde{w}\right\|_{L^{2}(\mathcal{Q})}^{2}\right)+\Xi_{\beta} \leq C_{26}|s| \int_{-\infty}^{\infty}\|\widetilde{w}\|_{L^{2}\left(\partial \mathcal{G}_{N}\right)}\left\|\partial_{y_{2}} \widetilde{w}\right\|_{L^{2}\left(\partial \mathcal{G}_{N}\right)} \mathrm{d} s \\
&+\|q\|_{L^{2}(\mathcal{Q})}^{2}+\|\widetilde{w}\|_{H^{1}(\mathcal{Q})}^{2}, \quad \forall|s| \geq s_{0}(\tau)
\end{aligned}
$$

with some constant $C_{27}>0$ and by $(5.27)$

$$
\left|\Xi_{\widetilde{\beta}}^{(2)}\right|+|s| \int_{-\infty}^{\infty}\|\widetilde{w}\|_{L^{2}\left(\partial \mathcal{G}_{N}\right)}\left\|\partial_{y_{2}} \widetilde{w}\right\|_{L^{2}\left(\partial \mathcal{G}_{N}\right)} \mathrm{d} s \leq \epsilon(\delta)\left\|\left(\frac{\partial \widetilde{w}}{\partial y_{2}}, \widetilde{w}\right)\right\|_{X}^{2}
$$


where we set

$$
\left\|\left(\frac{\partial \widetilde{w}}{\partial y_{2}}, \widetilde{w}\right)\right\|_{X}^{2}=\left\|h(s) \frac{\partial \widetilde{w}}{\partial y_{2}}\right\|_{L^{2}(\Sigma)}^{2}+\|h(s) \widetilde{w}\|_{L^{2}\left(\mathbb{R}^{1} ; H^{1}\left(\mathbb{R}^{2}\right)\right)}^{2}+\left\|h^{3}(s) \widetilde{w}\right\|_{L^{2}(\Sigma)}^{2}
$$

and the parameter $\epsilon(\delta) \rightarrow+0$ as $\delta \rightarrow+0$.

We set

$$
w_{1, \nu}=\mathcal{F}_{\sigma} \chi_{\nu}\left(D^{\prime}\right) v_{1}, \quad w_{2, \nu}=\mathcal{F}_{\sigma} \chi_{\nu}\left(D^{\prime}\right) v_{2} .
$$

Later we will need to apply (5.29) and (5.30) to the functions $w_{1, \nu}$ and $w_{2, \nu}$, since we would like to take the advantage of (5.23). However it is directly impossible because the condition $\operatorname{supp} \chi_{\nu}\left(D^{\prime}\right) \mathbf{v} \subset B_{\delta} \times \mathbb{R}^{1}$ does not hold true, in general. On the other hand, using the fact that

$$
\int_{\mathbb{R}^{2} \backslash B_{2 \delta}} \int_{\mathbb{R}^{1}} h^{4}(s) \sum_{|\alpha| \leq 2}\left|D^{\alpha} w_{j, \nu}\right|^{2} \mathrm{~d} y_{0} \mathrm{~d} y_{1} \mathrm{~d} s \leq C_{28}\|\mathbf{v}\|_{\left(H^{1}(\mathcal{Q})\right)^{2}}^{2},
$$

we can modify (5.29) and (5.30):

$$
\begin{aligned}
& C_{29}\left(\left\|h(s) w_{j(\beta), \nu}\right\|_{H^{1}(\mathcal{Q})}^{2}+\left\|h^{3}(s) w_{j(\beta), \nu}\right\|_{L^{2}(\mathcal{Q})}^{2}\right)+\Xi_{\beta} \\
\leq & \left\|P_{\beta, s} w_{j(\beta), \nu}\right\|_{L^{2}(\mathcal{Q})}^{2}+C_{30}\|\mathbf{v}\|_{\left(H^{1}(\mathcal{Q})\right)^{2}}^{2}+C_{30}|s| \int_{-\infty}^{\infty}\left\|w_{j(\beta), \nu}\right\|_{L^{2}\left(\partial \mathcal{G}_{N}\right)}\left\|\partial_{y_{2}} w_{j(\beta), \nu}\right\|_{L^{2}\left(\partial \mathcal{G}_{N}\right)} \mathrm{d} s
\end{aligned}
$$

where $C_{29}>0$ is independent of $s, N$ and we set $j(\beta)=1$ if $\beta=\mu$ and $j(\beta)=2$ if $\beta=\lambda+2 \mu$, and

$$
\left|\Xi_{\beta}^{(2)}\right|+|s| \int_{-\infty}^{\infty}\left\|w_{j(\beta), \nu}\right\|_{L^{2}\left(\partial \mathcal{G}_{N}\right)}\left\|\partial_{y_{2}} w_{j(\beta), \nu}\right\|_{L^{2}\left(\partial \mathcal{G}_{N}\right)} \mathrm{d} s \leq \epsilon\left\|\left(\frac{\partial w_{j(\beta), \nu}}{\partial y_{2}}, w_{j(\beta), \nu}\right)\right\|_{X}^{2}+C_{31}\|\mathbf{v}\|_{\left(H^{1}(\mathcal{Q})\right)^{2}}^{2}
$$

Now we will prove (5.15) separately in the cases: $r_{\mu}(\gamma)=0$ (Sect. 6), $r_{\lambda+2 \mu}(\gamma)=0$ (Sect. 7) and $r_{\mu}(\gamma) \neq 0$, $r_{\lambda+2 \mu}(\gamma) \neq 0$ (Sect. 8).

\section{THE CASE $r_{\mu}(\gamma)=0$}

In this section, we treat the case where $r_{\mu}(\gamma)=0$ with $\gamma=\left(y^{*}, \zeta^{*}\right) \equiv\left(y^{*}, s^{*}, \xi_{0}^{*}, \xi_{1}^{*}\right) \in \Sigma \times \mathbb{S}^{2}$. Let $\chi_{\nu}$ be a member of the partition of unity such that

$$
\operatorname{supp} \chi_{\nu} \subset \mathcal{O}\left(\delta_{1}\right) \equiv\left\{\zeta=\left(s, \zeta_{0}, \zeta_{1}\right) ;\left|\frac{\zeta}{|\zeta|}-\zeta^{*}\right|<\delta_{1}\right\} .
$$

We note that by (5.31) and (5.32), there exist $C_{1}>0$ and $C_{2}>0$ such that

$$
\begin{aligned}
C_{1}\left(\left\|h(s) w_{1, \nu}\right\|_{H^{1}(\mathcal{Q})}^{2}+\left\|h^{3}(s) w_{1, \nu}\right\|_{L^{2}(\mathcal{Q})}^{2}\right) & +\Xi_{\mu}^{(1)} \\
\leq & C_{2}\left(\left\|\mathbf{P}_{\mu} v_{1, \nu}\right\|_{L^{2}(\mathcal{Q})}^{2}+\left\|w_{1, \nu}\right\|_{H^{1}(\mathcal{Q})}^{2}\right)+\epsilon(\delta)\left\|\left(\frac{\partial w_{1, \nu}}{\partial y_{2}}, w_{1, \nu}\right)\right\|_{X}^{2},
\end{aligned}
$$


and the parameter $\epsilon$ can be taken sufficiently small, if we decrease $\delta$. Note that $\Xi_{\mu}^{(1)}$ can be written in the form

$$
\begin{aligned}
\Xi_{\mu}^{(1)}= & \int_{\Sigma}\left(|s| \mu^{2} \varphi_{y_{2}}\left(y^{*}\right)\left|\frac{\partial w_{1, \nu}}{\partial y_{2}}\right|^{2}+|s|^{3} \mu^{2} \varphi_{y_{2}}^{3}\left(y^{*}\right)\left|w_{1, \nu}\right|^{2}\right) \mathrm{d} \Sigma \\
& +\operatorname{Re} \int_{\Sigma} 2|s| \mu \frac{\partial w_{1, \nu}}{\partial y_{2}} \overline{\left(\mu \varphi_{y_{1}}\left(y^{*}\right) \frac{\partial w_{1, \nu}}{\partial y_{1}}-\varphi_{y_{0}}\left(y^{*}\right) \frac{\partial w_{1, \nu}}{\partial y_{0}}\right)} \mathrm{d} \Sigma \\
& +\int_{\Sigma}|s| \mu \varphi_{y_{2}}\left(y^{*}\right)\left(\xi_{0}^{2}-\mu \xi_{1}^{2}-s^{2} \varphi_{y_{0}}^{2}\left(y^{*}\right)+s^{2} \mu \varphi_{y_{1}}^{2}\left(y^{*}\right)\right)\left|\widehat{v}_{1, \nu}\right|^{2} \mathrm{~d} \Sigma \\
\equiv & J_{1}+J_{2}+J_{3} .
\end{aligned}
$$

Let us introduce the set $\mathcal{M}$ by formula

$$
\mathcal{M}=\left\{\zeta=\left(s, \xi_{0}, \xi_{1}\right) \in \mathbb{S}^{2} ; \frac{\mu}{2} \varphi_{y_{2}}\left(y^{*}\right) \widehat{C} s^{2}>4 \mu^{2} \frac{\varphi_{y_{1}}^{2}\left(y^{*}\right)}{\left|\varphi_{y_{2}}\left(y^{*}\right)\right|} \xi_{1}^{2}+4 \frac{\varphi_{y_{0}}^{2}\left(y^{*}\right)}{\left|\varphi_{y_{2}}\left(y^{*}\right)\right|} \xi_{0}^{2}+2 \mu^{2} \varphi_{y_{2}}\left(y^{*}\right)\left(\left|\xi_{0}\right|^{2}+\left|\xi_{1}\right|^{2}\right)\right\}
$$

where $\widehat{C}=-p_{\mu}\left(y^{*}, \nabla \varphi\left(y^{*}\right)\right)$. By $(2.6)$, it follows that $\widehat{C}$ is positive.

Next we introduce the set $\widetilde{\mathcal{M}}$ by the formula

$$
\widetilde{\mathcal{M}}=\left\{\zeta=\left(s, \xi_{0}, \xi_{1}\right) \in \mathbb{S}^{2} ; \frac{\mu}{4} \varphi_{y_{2}}\left(y^{*}\right) \widehat{C} s^{2}<4 \mu^{2} \frac{\varphi_{y_{1}}^{2}\left(y^{*}\right)}{\left|\varphi_{y_{2}}\left(y^{*}\right)\right|} \xi_{1}^{2}+4 \frac{\varphi_{y_{0}}^{2}\left(y^{*}\right)}{\left|\varphi_{y_{2}}\left(y^{*}\right)\right|} \xi_{0}^{2}+2 \mu^{2} \varphi_{y_{2}}\left(y^{*}\right)\left(\left|\xi_{0}\right|^{2}+\left|\xi_{1}\right|^{2}\right)\right\}
$$

Then we can see that $\mathbb{S}^{2} \subset \mathcal{M} \cup \widetilde{\mathcal{M}}$. Therefore, taking the parameter $\delta_{1}$ sufficiently small, we obtain either $\mathcal{O}\left(\delta_{1}\right) \subset \mathcal{M}$ or $\mathcal{O}\left(\delta_{1}\right) \subset \widetilde{M}$. The main purpose of this section is the proof of the following lemma.

Lemma 6.1. If $\gamma=\left(y^{*}, \zeta^{*}\right)$ is a point on $\Sigma \times \mathbb{S}^{2}$ such that $r_{\mu}(\gamma)=0$ and $\operatorname{supp} \chi_{\nu} \subset \mathcal{O}\left(\delta_{1}\right) \subset \widetilde{\mathcal{M}}$, then estimate (5.15) holds true. If $\gamma=\left(y^{*}, \zeta^{*}\right) \in \mathcal{M}$, then estimate (5.15) holds true also.

Proof. We consider two cases.

Case A. Assume that $\operatorname{supp} \widehat{\mathbf{v}}_{\nu} \subset \mathcal{O}\left(\delta_{1}\right) \subset \mathcal{M}$.

Applying the Cauchy-Bunyakovskii inequality and using (6.3) and (2.6), we see that there exists a constant $C_{3}>0$ such that

$$
\begin{aligned}
\Xi_{\mu}^{(1)} \geq & \int_{\Sigma}\left(|s| \mu^{2} \varphi_{y_{2}}\left(y^{*}\right)\left|\frac{\partial w_{1, \nu}}{\partial y_{2}}\right|^{2}-|s|^{3} \mu \varphi_{y_{2}}\left(y^{*}\right) p_{\mu}\left(y^{*}, \nabla \varphi\left(y^{*}\right)\right)\left|w_{1, \nu}\right|^{2}\right) \mathrm{d} \Sigma \\
& -\int_{\Sigma}\left(\frac{1}{2}|s| \mu^{2} \varphi_{y_{2}}\left(y^{*}\right)\left|\frac{\partial w_{1, \nu}}{\partial y_{2}}\right|^{2}+4|s| \mu^{2} \frac{\varphi_{y_{1}}^{2}\left(y^{*}\right)}{\left|\varphi_{y_{2}}\left(y^{*}\right)\right|}\left|\frac{\partial w_{1, \nu}}{\partial y_{1}}\right|^{2}+4|s| \frac{\varphi_{y_{0}}^{2}\left(y^{*}\right)}{\left|\varphi_{y_{2}}\left(y^{*}\right)\right|}\left|\frac{\partial w_{1, \nu}}{\partial y_{0}}\right|^{2}\right) \mathrm{d} \Sigma \\
& -\int_{\Sigma}|s| \mu^{2} \varphi_{y_{2}}\left(y^{*}\right) \xi_{1}^{2}\left|\widehat{v}_{1, \nu}\right|^{2} \mathrm{~d} \Sigma \\
\geq & C_{3} \int_{\Sigma}\left(\frac{1}{2}|s| \mu^{2} \varphi_{y_{2}}\left(y^{*}\right)\left|\frac{\partial w_{1, \nu}}{\partial y_{2}}\right|^{2}+|s|\left|\frac{\partial w_{1, \nu}}{\partial y_{1}}\right|^{2}+|s|\left|\frac{\partial w_{1, \nu}}{\partial y_{0}}\right|^{2}+\frac{1}{2}|s|^{3} \mu \varphi_{y_{2}}\left(y^{*}\right) \widehat{C}\left|w_{1, \nu}\right|^{2}\right) \mathrm{d} \Sigma
\end{aligned}
$$

Similary we have

$$
\Xi_{\lambda+2 \mu}^{(1)} \geq C_{4} \int_{\Sigma}\left\{|s|\left(\left|\frac{\partial w_{2, \nu}}{\partial y_{2}}\right|^{2}+\left|\frac{\partial w_{2, \nu}}{\partial y_{1}}\right|^{2}+\left|\frac{\partial w_{2, \nu}}{\partial y_{0}}\right|^{2}\right)+|s|^{3}\left|w_{2, \nu}\right|^{2}\right\} \mathrm{d} \Sigma .
$$


Combining (6.4) and (6.5), we obtain

$$
\Xi_{\mu}^{(1)}+\Xi_{\lambda+2 \mu}^{(1)} \geq C_{5}\left\|\left(\frac{\partial w_{\nu}}{\partial y_{2}}, w_{\nu}\right)\right\|_{X}^{2} .
$$

If we apply (5.31) with $\beta=\lambda+2 \mu$, then (6.1), (6.4) and (6.6) imply (5.15).

Case B. Assume that supp $\widehat{\mathbf{v}}_{\nu} \subset \widetilde{\mathcal{M}}$.

By (5.18)-(5.20), there exists $C_{6}>0$ such that

$$
\begin{aligned}
\left|\xi_{0}^{2}-s^{2} \varphi_{y_{0}}^{2}\left(y^{*}\right)-\mu \xi_{1}^{2}+\mu s^{2} \varphi_{y_{1}}^{2}\left(y^{*}\right)\right|+\left|\xi_{0} s \varphi_{y_{0}}\left(y^{*}\right)-\mu s \xi_{1} \varphi_{y_{1}}\left(y^{*}\right)\right| & \\
& \leq \delta_{1} C_{6}\left(\left|\xi_{1}\right|^{2}+\left|\xi_{0}\right|^{2}+s^{2}\right), \quad \forall \zeta \in \mathcal{O}\left(\delta_{1}\right) .
\end{aligned}
$$

Now we suppose that the parameter $\delta_{1}$ is sufficiently small such that there exists a constant $C_{7}>0$ such that

$$
\left|\xi_{0}\right|^{2} \leq C_{7}\left(\left|\xi_{1}\right|^{2}+s^{2}\right), \quad \forall \zeta \in \mathcal{O}\left(\delta_{1}\right) .
$$

Then, by (6.7), we have

$$
\left|J_{3}\right| \leq \delta_{1} \mu \varphi_{y_{2}}\left(y^{*}\right)\left\|\left(\frac{\partial w_{1, \nu}}{\partial y_{2}}, w_{1, \nu}\right)\right\|_{X}^{2} .
$$

Moreover we claim that there exists $\delta_{0}>0$ such that if $\delta_{1} \in\left(0, \delta_{0}\right)$, then there exists $C_{8}>0$ such that

$$
\left|\xi_{0}\right| \leq C_{8}\left|\xi_{1}\right|, \quad \forall \zeta \in \mathcal{O}\left(\delta_{1}\right)
$$

Our proof is by contradiction. Suppose that (6.10) is not true. Then for the sequence $\delta_{1}(n)=\frac{1}{n}$, there exists a sequence $\left(\xi_{0}(n), \xi_{1}(n)\right) \rightarrow\left(\xi_{0}^{*}, \xi_{1}^{*}\right)$ such that $\xi_{1}(n) / \xi_{0}(n) \rightarrow 0$. Hence for $\zeta^{*}$ we have $r_{\mu}\left(y^{*}, \zeta^{*}\right)=0$, and $\xi_{1}^{*}=0, \xi_{0}^{*} \neq 0$ by the definition of the set $\widetilde{\mathcal{M}}$. Therefore $s^{*} \varphi_{y_{0}}\left(y^{*}\right)=0$. If $s^{*}=0$, then we obtain $\left(\xi_{0}^{*}\right)^{2}=0$ and if $\varphi_{y_{0}}\left(y^{*}\right)=0$, then $\left(\xi_{0}^{*}\right)^{2}+\mu \varphi_{y_{1}}^{2}\left(y^{*}\right)\left(s^{*}\right)^{2}=0$ by (5.19), (5.20). Therefore in the both cases, we have the equality $\xi_{0}^{*}=0$ which leads us to a contradiction.

Note that if $r_{\lambda+2 \mu}(\gamma)=0$, then

$$
\varphi_{y_{0}}\left(y^{*}\right)=0, \quad \varphi_{y_{1}}\left(y^{*}\right)=0, \quad \xi_{0}^{*}=\xi_{1}^{*}=0, \quad s^{*}=1
$$

and the conic neighbourhood of $\zeta^{*}$ is in the set $\mathcal{M}$ provided that the parameter $\delta_{1}$ is chosen sufficiently small. Therefore if $\gamma \in \widetilde{\mathcal{M}}$ and $r_{\mu}(\gamma)=0$, then we have $r_{\lambda+2 \mu}(\gamma) \neq 0$ and by Proposition 5.4, decomposition (5.23) holds true. We set $V_{\lambda+2 \mu}^{+}=\left(D_{y_{2}}-\Gamma_{\lambda+2 \mu}^{+}\left(y, D^{\prime}\right)\right) v_{2, \nu}$. Then

$$
\mathbf{P}_{\lambda+2 \mu} v_{2, \nu}=(\lambda+2 \mu)|G|^{2}\left(D_{y_{2}}-\Gamma_{\lambda+2 \mu}^{-}\left(y, D^{\prime}\right)\right) V_{\lambda+2 \mu}^{+}+T_{\lambda+2 \mu} v_{2, \nu},
$$

where $T_{\lambda+2 \mu} \in \mathcal{L}\left(H^{1}(\mathcal{Q}), L^{2}(\mathcal{Q})\right)$. This decomposition and Proposition 5.4 immediately imply

$$
\left\|\left.h\left(D_{\sigma}\right)\left(D_{y_{2}}-\Gamma_{\lambda+2 \mu}^{+}\left(y, D^{\prime}\right)\right) v_{2, \nu}\right|_{y_{2}=0}\right\|_{L^{2}(\Sigma)} \leq C_{9}\left(\left\|P_{\lambda+2 \mu, s} w_{2, \nu}\right\|_{L^{2}(\mathcal{Q})}+\|\mathbf{v}\|_{\left(H^{1}(\mathcal{Q})\right)^{2}}\right) .
$$

Now we need again obtain the estimate of $\Xi_{\mu}^{(1)}$. We start from the term $J_{2}$. By (5.16), we have

$$
\begin{aligned}
J_{2}=\operatorname{Re} \int_{\Sigma} 2|s|(\lambda+2 \mu)\left(\frac{\partial w_{2, \nu}}{\partial y_{1}}-|s| \varphi_{y_{1}}\left(y^{*}\right) w_{2, \nu}\right) \times \overline{\left(\mu \frac{\partial w_{1, \nu}}{\partial y_{1}} \varphi_{y_{1}}\left(y^{*}\right)-\frac{\partial w_{1, \nu}}{\partial y_{0}} \varphi_{y_{0}}\left(y^{*}\right)\right)} \mathrm{d} \Sigma \\
+\operatorname{Re} \int_{\Sigma} 2|s| \mu\left(|s| \varphi_{y_{2}}\left(y^{*}\right) w_{1, \nu}-g_{1, \nu}\right) \overline{\left(\mu \frac{\partial w_{1, \nu}}{\partial y_{1}} \varphi_{y_{1}}\left(y^{*}\right)-\frac{\partial w_{1, \nu}}{\partial y_{0}} \varphi_{y_{0}}\left(y^{*}\right)\right)} \mathrm{d} \Sigma
\end{aligned}
$$


and denoting

$$
\begin{aligned}
\tilde{\alpha}_{\lambda+2 \mu}^{+}\left(y^{\prime}, D\right) & =\alpha_{\lambda+2 \mu}^{+}\left(y^{\prime}, D\right)+i\left|D_{\sigma}\right|\left(\varphi_{y_{2}}-\varphi_{y_{2}}\left(y^{*}\right)\right) \\
& -\frac{\mu}{\lambda+2 \mu}\left(\frac{\partial v_{1, \nu}}{\partial y_{1}}-\left|D_{\sigma}\right| \varphi_{y_{1}}\left(y^{*}\right) v_{1, \nu}\right)-i \tilde{\alpha}_{\lambda+2 \mu}^{+}\left(y, D^{\prime}\right) v_{2, \nu}=i V_{\lambda+2 \mu}^{+}(\cdot, 0)-\frac{\mu}{\lambda+2 \mu} \mathcal{F}_{\sigma}^{-1} g_{2, \nu} .
\end{aligned}
$$

Here and henceforth $\left|D_{\sigma}\right|$ is the pseudo-differential operator with the symbol $|s|$.

First assume that $s^{*}=0$. Then we can see by $\left|s^{*}\right|^{2}+\left|\xi_{0}^{*}\right|^{2}+\left|\xi_{1}^{*}\right|^{2}=1$ that $\left|\tilde{\alpha}_{\lambda+2 \mu}^{+}(\gamma)\right|=\left|r_{\lambda+2 \mu}(\gamma)\right| \neq 0$. Therefore, by Proposition 5.2.A from [47], p. 105, there exists a parametrix of the operator $\tilde{\alpha}_{\lambda+2 \mu}^{+}\left(y, D^{\prime}\right)$ which we denote by $\left(\tilde{\alpha}_{\lambda+2 \mu}^{+}\left(y, D^{\prime}\right)\right)^{-1}$. From $(6.13)$ we obtain

$$
v_{2, \nu}=-\frac{1}{i}\left(\tilde{\alpha}_{\lambda+2 \mu}^{+}\left(y, D^{\prime}\right)\right)^{-1}\left(\frac{\mu}{\lambda+2 \mu}\left(\frac{\partial v_{1, \nu}}{\partial y_{1}}-\left|D_{\sigma}\right| \varphi_{y_{1}}\left(y^{*}\right) v_{1, \nu}\right)+i V_{\lambda+2 \mu}^{+}(\cdot, 0)-\frac{\mu}{\lambda+2 \mu} \mathcal{F}_{\sigma}^{-1} g_{2, \nu}\right)+T_{0} v_{2, \nu}
$$

where $T_{0} \in \mathcal{L}\left(L^{2}(\Sigma), H^{1}(\Sigma)\right)$. Using (6.14), we transform (6.12) to obtain

$$
\begin{aligned}
J_{2}=\operatorname{Re} \int_{\Sigma}-\frac{2\left|D_{\sigma}\right| \mu}{i}\left(\frac{\partial}{\partial y_{1}}-\left|D_{\sigma}\right| \varphi_{y_{1}}\left(y^{*}\right)\right)\left(\tilde{\alpha}_{\lambda+2 \mu}^{+}\left(y, D^{\prime}\right)\right)^{-1} \\
\quad\left(\frac{\partial v_{1, \nu}}{\partial y_{1}}-\left|D_{\sigma}\right| \varphi_{y_{1}}\left(y^{*}\right) v_{1, \nu}\right) \overline{\left(\mu \frac{\partial v_{1, \nu}}{\partial y_{1}} \varphi_{y_{1}}\left(y^{*}\right)-\frac{\partial v_{1, \nu}}{\partial y_{0}} \varphi_{y_{0}}\left(y^{*}\right)\right)} \mathrm{d} \Sigma+\kappa_{3},
\end{aligned}
$$

where

$$
\begin{aligned}
\kappa_{3}= & \operatorname{Re} \int_{\Sigma} 2\left|D_{\sigma}\right| \mu\left(\left|D_{\sigma}\right| \varphi_{y_{2}}\left(y^{*}\right) v_{1, \nu}+\mathcal{F}_{\sigma}^{-1} g_{1, \nu}\right) \overline{\left(\mu \frac{\partial v_{1, \nu}}{\partial y_{1}} \varphi_{y_{1}}\left(y^{*}\right)-\frac{\partial v_{1, \nu}}{\partial y_{0}} \varphi_{y_{0}}\left(y^{*}\right)\right)} \mathrm{d} \Sigma \\
& +\operatorname{Re} \int_{\Sigma} 2\left|D_{\sigma}\right|(\lambda+2 \mu)\left(\frac{\partial}{\partial y_{1}}-|s| \varphi_{y_{1}}\left(y^{*}\right)\right) \\
\times & {\left[-\frac{1}{i}\left(\tilde{\alpha}_{\lambda+2 \mu}^{+}\left(y, D^{\prime}\right)\right)^{-1}\left(i V_{\lambda+2 \mu}^{+}(\cdot, 0)-\frac{\mu}{\lambda+2 \mu} \mathcal{F}_{\sigma}^{-1} g_{2, \nu}\right)+T_{0} v_{2, \nu}\right] \times \overline{\left(\mu \frac{\partial v_{1, \nu}}{\partial y_{1}} \varphi_{y_{1}}\left(y^{*}\right)-\frac{\partial v_{1, \nu}}{\partial y_{0}} \varphi_{y_{0}}\left(y^{*}\right)\right)} \mathrm{d} \Sigma . }
\end{aligned}
$$

Then we have

$$
\left|\kappa_{3}\right| \leq \epsilon\left\|\left(\frac{\partial \mathbf{w}_{\nu}}{\partial y_{2}}, \mathbf{w}_{\nu}\right)\right\|_{X}^{2}+C_{10}\left(\|h(s) \mathbf{g}\|_{\left(L^{2}(\Sigma)\right)^{2}}^{2}+\left\|P_{\lambda+2 \mu, s} w_{2, \nu}\right\|_{L^{2}(\mathcal{Q})}^{2}\right)
$$

and $\epsilon$ can be chosen arbitrarily small by taking $\delta$ small enough.

Let us consider the pseudo-differential operator

$$
b\left(y, D^{\prime}\right) \equiv \frac{1}{i}\left(\frac{\partial}{\partial y_{1}}-|s| \varphi_{y_{1}}\left(y^{*}\right)\right)\left(\tilde{\alpha}_{\lambda+2 \mu}^{+}\left(y, D^{\prime}\right)\right)^{-1} .
$$

By (6.7), for the principal symbol of this operator, we have

$$
\begin{aligned}
b\left(y^{*}, \zeta\right) & =\frac{1}{i}\left(i \xi_{1}-|s| \varphi_{y_{1}}\left(y^{*}\right)\right)\left(\tilde{\alpha}_{\lambda+2 \mu}^{+}\left(y^{*}, \zeta\right)\right)^{-1} \\
& \equiv-\operatorname{sign}\left(\xi_{1}^{*} \sqrt{\left(\frac{\lambda+\mu}{\lambda+2 \mu}\right)\left(y^{*}\right)} \frac{\left(i \xi_{1}-|s| \varphi_{y_{1}}\left(y^{*}\right)\right)}{\xi_{1}+i|s| \varphi_{y_{1}}\left(y^{*}\right)}+\widetilde{b}\left(y^{*}, \zeta\right)\right. \\
& =\frac{1}{i} \sqrt{\left(\frac{\lambda+\mu}{\lambda+2 \mu}\right)\left(y^{*}\right)}+\widetilde{b}\left(y^{*}, \zeta\right),
\end{aligned}
$$


where $\widetilde{b}\left(y^{*}, \xi^{*}\right)=0$. Therefore the operator $b\left(y, D^{\prime}\right)$ can be represented in the form

$$
b\left(y, D^{\prime}\right)=\frac{1}{i} \sqrt{\frac{\lambda+\mu}{\lambda+2 \mu}(y)}+\widetilde{b}\left(y, D^{\prime}\right)
$$

where $\widetilde{b}\left(y, D^{\prime}\right) \in \mathcal{L}\left(L^{2}(\Sigma), L^{2}(\Sigma)\right)$ and

$$
\left\|\widetilde{b}\left(y, D^{\prime}\right)\right\|_{\mathcal{L}\left(L^{2}(\Sigma), L^{2}(\Sigma)\right)} \leq \epsilon .
$$

Using (6.17) in (6.15), we obtain

$$
\begin{aligned}
& J_{2}=\operatorname{Re} \int_{\Sigma}-2\left|D_{\sigma}\right| \mu\left(\frac{\operatorname{sign}\left(\xi_{1}^{*}\right)}{i} \sqrt{\left(\frac{\lambda+\mu}{\lambda+2 \mu}\right)\left(y^{*}\right)}+\widetilde{b}\left(y, D^{\prime}\right)\right)\left(\frac{\partial v_{1, \nu}}{\partial y_{1}}-\left|D_{\sigma}\right| \varphi_{y_{1}}\left(y^{*}\right) v_{1, \nu}\right) \\
& \times \overline{\left(\mu \frac{\partial v_{1, \nu}}{\partial y_{1}} \varphi_{y_{1}}\left(y^{*}\right)-\frac{\partial v_{1, \nu}}{\partial y_{0}} \varphi_{y_{0}}\left(y^{*}\right)\right)} d \Sigma+\kappa_{3} \\
& =\operatorname{Re} \int_{\Sigma}-2\left|D_{\sigma}\right| \mu\left(\widetilde{b}\left(y, D^{\prime}\right)+\frac{\operatorname{sign}\left(\xi_{1}^{*}\right)}{i} \sqrt{\left(\frac{\lambda+\mu}{\lambda+2 \mu}\right)\left(y^{*}\right)}\right)\left(\frac{\partial v_{1, \nu}}{\partial y_{1}}-\left|D_{\sigma}\right| \varphi_{y_{1}}\left(y^{*}\right) v_{1, \nu}\right) \\
& \times \overline{\left(\mu \frac{\partial v_{1, \nu}}{\partial y_{1}} \varphi_{y_{1}}\left(y^{*}\right)-\frac{\partial v_{1, \nu}}{\partial y_{0}} \varphi_{y_{0}}\left(y^{*}\right)\right)} \mathrm{d} \Sigma+\operatorname{Re} \kappa_{3} .
\end{aligned}
$$

By (6.7), (6.16) and (6.18), taking the parameters $\delta, \delta_{1}$ sufficiently small, we obtain

$$
\left|J_{2}\right| \leq \epsilon\left(\delta, \delta_{1}\right)\left\|\left(\frac{\partial \mathbf{w}_{\nu}}{\partial y_{2}}, \mathbf{w}_{\nu}\right)\right\|_{X}^{2}+C_{11}\left(\|h(s) \mathbf{g}\|_{\left(L^{2}(\Sigma)\right)^{2}}^{2}+\left\|P_{\lambda+2 \mu, s} w_{2, \nu}\right\|_{L^{2}(\mathcal{Q})}^{2}+\|\mathbf{v}\|_{\left(H^{1}(\mathcal{Q})\right)^{2}}^{2}\right),
$$

and $\epsilon\left(\delta, \delta_{1}\right) \rightarrow 0$ as $|\delta|+\left|\delta_{1}\right| \rightarrow 0$.

Next assume that $s^{*} \neq 0$. Then by (6.7) we have

$$
\left|\mu\left(y^{*}\right) \varphi_{y_{1}}\left(y^{*}\right) \xi_{1}-\varphi_{y_{0}}\left(y^{*}\right) \xi_{0}\right| \leq C \delta_{1}|\zeta|, \quad \forall \zeta \in \mathcal{O}\left(\delta_{1}\right)
$$

and (6.19) follows immediately. Therefore, for any $s^{*} \in \mathbb{R}^{1}$, by (6.1), (6.2), (6.9) and (6.19), we have

$$
\begin{gathered}
\int_{\Sigma}\left(h^{2}(s) \mu^{2} \varphi_{y_{2}}\left(y^{*}\right)\left|\frac{\partial w_{1, \nu}}{\partial y_{2}}\right|^{2}+h^{6}(s) \mu^{2} \varphi_{y_{2}}^{3}\left(y^{*}\right)\left|w_{1, \nu}\right|^{2}\right) \mathrm{d} \Sigma+C_{12}\left(\left\|h(s) w_{1, \nu}\right\|_{H^{1}(\mathcal{Q})}^{2}+\left\|h^{3}(s) w_{1, \nu}\right\|_{L^{2}(\mathcal{Q})}^{2}\right) \\
\leq C_{13}\left(\left\|P_{\lambda+2 \mu, s} w_{2, \nu}\right\|_{L^{2}(\mathcal{Q})}^{2}+\|h(s) \mathbf{g}\|_{\left(L^{2}(\Sigma)\right)^{2}}^{2}+\|\mathbf{v}\|_{\left(H^{1}(\mathcal{Q})\right)^{2}}^{2}+\epsilon\left\|\left(\frac{\partial \mathbf{w}_{\nu}}{\partial y_{2}}, \mathbf{w}_{\nu}\right)\right\|_{X}^{2}\right.
\end{gathered}
$$

From (5.16), we obtain

$$
\begin{aligned}
\int_{\Sigma}\left(|s|\left|\frac{\partial w_{2, \nu}}{\partial y_{1}}\right|^{2}\right. & \left.+|s|^{3} \mu^{2} \varphi_{y_{1}}^{2}\left(y^{*}\right)\left|w_{2, \nu}\right|^{2}\right) \mathrm{d} \Sigma \\
& \leq C_{14} \int_{\Sigma}\left(|s| \mu^{2} \varphi_{y_{2}}\left(y^{*}\right)\left|\frac{\partial w_{1, \nu}}{\partial y_{2}}\right|^{2}+|s|^{3} \mu^{2} \varphi_{y_{2}}^{3}\left(y^{*}\right)\left|w_{1, \nu}\right|^{2}\right) \mathrm{d} \Sigma+C_{14}\left\|h(s) \mathbf{g}_{\nu}\right\|_{\left(L^{2}(\Sigma)\right)^{2}}^{2}
\end{aligned}
$$


Using $(6.10),(6.21)$ and the definition of the set $\widetilde{\mathcal{M}}$, we obtain

$$
\begin{aligned}
& \int_{\Sigma}\left(h^{2}(s)\left|\frac{\partial w_{2, \nu}}{\partial y_{1}}\right|^{2}+h^{2}(s)\left|\frac{\partial w_{2, \nu}}{\partial y_{0}}\right|^{2}+h^{6}(s)\left|w_{2, \nu}\right|^{2}\right) \mathrm{d} \Sigma \\
& \quad \leq C_{15}\left\{\int_{\Sigma}\left(|s| \mu^{2} \varphi_{y_{2}}\left(y^{*}\right)\left|\frac{\partial w_{1, \nu}}{\partial y_{2}}\right|^{2}+|s|^{3} \mu^{2} \varphi_{y_{2}}^{3}\left(y^{*}\right)\left|w_{1, \nu}\right|^{2}\right) \mathrm{d} \Sigma+\epsilon\left(\sigma_{0}\right)\left\|\left(\frac{\partial \mathbf{w}_{\nu}}{\partial y_{2}}, \mathbf{w}_{\nu}\right)\right\|_{X}^{2}+\left\|h(s) \mathbf{g}_{\nu}\right\|_{\left(L^{2}(\Sigma)\right)^{2}}^{2}\right\}
\end{aligned}
$$

From (6.11) and (6.22), we have

$$
\begin{aligned}
& \int_{\Sigma} h^{2}(s)\left|\frac{\partial w_{2, \nu}}{\partial y_{2}}\right|^{2} \mathrm{~d} \Sigma \\
& \leq C_{16}\left\{\int_{\Sigma}\left(h^{2}(s)\left|\frac{\partial w_{2, \nu}}{\partial y_{1}}\right|^{2}+h^{2}(s)\left|\frac{\partial w_{2, \nu}}{\partial y_{0}}\right|^{2}+h^{6}(s)\left|w_{2, \nu}\right|^{2}\right) \mathrm{d} \Sigma\right. \\
& \left.+\left\|V_{\lambda+2 \mu}^{+}(\cdot, 0)\right\|_{L^{2}(\Sigma)}^{2}+\epsilon\left(\sigma_{0}\right)\left\|\left(\frac{\partial \mathbf{w}_{\nu}}{\partial y_{2}}, \mathbf{w}_{\nu}\right)\right\|_{X}^{2}+\left\|h(s) \mathbf{g}_{\nu}\right\|_{\left(L^{2}(\Sigma)\right)^{2}}^{2}\right\} \\
& \leq C_{17}\left\{\int_{\Sigma}\left(h^{2}(s)\left|\frac{\partial w_{1, \nu}}{\partial y_{2}}\right|^{2}+h^{6}(s)\left|w_{1, \nu}\right|^{2}\right) \mathrm{d} \Sigma+\left\|h(s) \mathbf{g}_{\nu}\right\|_{\left(L^{2}(\Sigma)\right)^{2}}^{2}\right. \\
& \left.\quad+\|\mathbf{v}\|_{\left(H^{1}(\mathcal{Q})\right)^{2}}^{2}+\left\|P_{\lambda+2 \mu, s} w_{2, \nu}\right\|_{L^{2}(\mathcal{Q})}^{2}+\epsilon\left(\sigma_{0}\right)\left\|\left(\frac{\partial \mathbf{w}_{\nu}}{\partial y_{2}}, \mathbf{w}_{\nu}\right)\right\|_{X}^{2}\right\}
\end{aligned}
$$

Finally (5.16), (6.10), (6.20) and (6.23) imply

$$
\begin{aligned}
& \int_{\Sigma} h^{2}(s)\left(\left|\frac{\partial w_{1, \nu}}{\partial y_{1}}\right|^{2}+\left|\frac{\partial w_{1, \nu}}{\partial y_{0}}\right|^{2}\right) \mathrm{d} \Sigma \\
& \leq C_{18}\left\{\int_{\Sigma}\left(h^{2}(s)\left|\frac{\partial w_{1, \nu}}{\partial y_{2}}\right|^{2}+h^{6}(s)\left|w_{1, \nu}\right|^{2}\right) \mathrm{d} \Sigma+\left\|h(s) \mathbf{g}_{\nu}\right\|_{\left(L^{2}(\Sigma)\right)^{2}}^{2}\right. \\
& \left.\quad+\|\mathbf{v}\|_{\left(H^{1}(\mathcal{Q})\right)^{2}}^{2}+\left\|P_{\lambda+2 \mu, s} w_{2, \nu}\right\|_{L^{2}(\mathcal{Q})}^{2}+\epsilon\left(\sigma_{0}\right)\left\|\left(\frac{\partial \mathbf{w}_{\nu}}{\partial y_{2}}, \mathbf{w}_{\nu}\right)\right\|_{X}^{2}\right)
\end{aligned}
$$

Inequalities (6.1), (6.20)-(6.24) imply

$$
\begin{aligned}
\left\|\left(\frac{\partial \mathbf{w}_{\nu}}{\partial y_{2}}, \mathbf{w}_{\nu}\right)\right\|_{X}^{2}+\left\|h(s) w_{1, \nu}\right\|_{H^{1}(\mathcal{Q})}^{2}+\left\|h^{3}(s) w_{1, \nu}\right\|_{L^{2}(\mathcal{Q})}^{2} \leq \epsilon\left\|\left(\frac{\partial \mathbf{w}_{\nu}}{\partial y_{2}}, \mathbf{w}_{\nu}\right)\right\|_{X}^{2} \\
+C_{19}\left(\|\mathbf{v}\|_{\left(H^{1}(\mathcal{Q})\right)^{2}}^{2}+\left\|h(s) \mathbf{g}_{\nu}\right\|_{\left(L^{2}(\Sigma)\right)^{2}}^{2}+\left\|P_{\mu, s} w_{2, \nu}\right\|_{L^{2}(\mathcal{Q})}^{2}+\left\|P_{\lambda+2 \mu, s} w_{2, \nu}\right\|_{L^{2}(\mathcal{Q})}^{2}\right)
\end{aligned}
$$

From this inequality and (5.31), (5.32) with $\beta=\lambda+2 \mu$, we obtain (5.15). Thus the proof of Lemma 6.1 is complete. 


\section{The CASE $r_{\lambda+2 \mu}(\gamma)=0$}

In this section, we will prove

Lemma 7.1. Let $\gamma=\left(y^{*}, \zeta^{*}\right)$ be a point on $\Sigma \times \mathbb{S}^{2}$ such that $r_{\lambda+2 \mu}(\gamma)=0$. If $\operatorname{supp} \chi_{\nu} \subset \mathcal{O}\left(\delta_{1}\right) \subset \widetilde{\mathcal{M}}$, then estimate (5.15) holds true.

Proof. We note that if $r_{\mu}(\gamma)=0$, then $s^{*} \neq 0$ and $\xi_{0}^{*}=\xi_{1}^{*}=\varphi_{y_{0}}\left(y^{*}\right)=\varphi_{y_{1}}\left(y^{*}\right)=0$. Consequently $\zeta^{*} \in \mathcal{M}$ and this case was treated in the previous section. Therefore, taking the parameters $\delta$ and $\delta_{1}$ sufficiently small, we may assume that there exists a constant $\widehat{C}>0$ such that

$$
\left|r_{\mu}(y, \zeta)\right| \geq \widehat{C}|\zeta|^{2}, \quad \forall(y, \zeta) \in B_{\delta} \times \mathcal{O}\left(\delta_{1}\right),|\zeta| \geq 1
$$

By (5.19) and (5.20), there exist $\delta_{0}>0$ and $C_{1}>0$ such that for all $\delta_{1} \in\left(0, \delta_{0}\right)$ we have

$$
\left|\xi_{0}\right|^{2} \leq C_{1}\left(\xi_{1}^{2}+s^{2}\right), \quad \forall \zeta \in \mathcal{O}\left(\delta_{1}\right)
$$

We consider the following three cases.

Case A. Assume that $s^{*}=0$ and

$$
\mu\left(y^{*}\right) \varphi_{y_{2}}\left(y^{*}\right)>\frac{\left|\mu\left(y^{*}\right) \varphi_{y_{1}}\left(y^{*}\right) \xi_{1}^{*}-\varphi_{y_{0}}\left(y^{*}\right) \xi_{0}^{*}\right|}{\sqrt{\frac{\lambda+\mu}{\mu}\left(y^{*}\right)}\left|\xi_{1}^{*}\right|} .
$$

In that case, there exists a constant $C_{2}>0$ such that

$$
-\operatorname{Im} \Gamma_{\mu}^{ \pm}(y, \zeta) \geq C_{2}|s|, \quad \forall(y, \zeta) \in B_{\delta} \times \mathcal{O}\left(\delta_{1}\right),
$$

provided that $|\delta|+\left|\delta_{1}\right|$ is sufficiently small. Since $s^{*}=0$, we may assume that for some constant $C_{3}>0$,

$$
\left|\xi_{0}\right|^{2}+s^{2} \leq C_{3} \xi_{1}^{2}, \quad \forall \zeta \in \mathcal{O}\left(\delta_{1}\right)
$$

taking a sufficiently small $\delta_{1}$. We set $V_{\mu}^{ \pm}=\left(D_{y_{2}}-\Gamma_{\mu}^{ \pm}\left(y, D^{\prime}\right)\right) v_{1, \nu}$. Then, by Proposition 5.3,

$$
\mathbf{P}_{\mu} v_{1, \nu}=|G|^{2} \mu\left(D_{y_{2}}-\Gamma_{\mu}^{\mp}\left(y, D^{\prime}\right)\right) V_{\mu}^{ \pm}+T_{\mu}^{ \pm} v_{1, \nu}
$$

where $T_{\mu}^{ \pm} \in \mathcal{L}\left(H^{1}(\mathcal{Q}), L^{2}(\mathcal{Q})\right)$. This decomposition and Proposition 5.4 imply

$$
\left\|\left.h\left(D_{\sigma}\right)\left(D_{y_{2}}-\Gamma_{\mu}^{ \pm}\left(y, D^{\prime}\right)\right) v_{1, \nu}\right|_{y_{2}=0}\right\|_{L^{2}(\Sigma)} \leq C_{4}\left(\left\|\mathbf{P}_{\mu} v_{1, \nu}\right\|_{L^{2}(\mathcal{Q})}+\|\mathbf{v}\|_{\left(H^{1}(\mathcal{Q})\right)^{2}}\right)
$$

We have

$$
-V_{\mu}^{+}(\cdot, 0)+V_{\mu}^{-}(\cdot, 0)=\left(\alpha_{\mu}^{+}\left(y, D^{\prime}\right)-\alpha_{\mu}^{-}\left(y, D^{\prime}\right)\right) v_{1, \nu} \quad \text { on } \Sigma .
$$

Since $\alpha_{\mu}^{+}\left(y^{*}, \zeta^{*}\right)-\alpha_{\mu}^{-}\left(y^{*}, \zeta^{*}\right)=2 \sqrt{r_{\mu}\left(y^{*}, \zeta^{*}\right)} \neq 0$, by (7.4), (7.5) and Gårding's inequality, we have

$$
\int_{\Sigma}\left(h^{2}(s)\left(\left|\frac{\partial w_{1, \nu}}{\partial y_{1}}\right|^{2}+\left|\frac{\partial w_{1, \nu}}{\partial y_{0}}\right|^{2}\right)+h^{6}(s)\left|w_{1, \nu}\right|^{2}\right) \mathrm{d} \Sigma \leq C_{5}\left(\left\|P_{\mu, s} w_{1, \nu}\right\|_{L^{2}(\mathcal{Q})}^{2}+\|\mathbf{v}\|_{\left(H^{1}(\mathcal{Q})\right)^{2}}^{2} .\right.
$$

By (7.6) and (7.4), we obtain

$$
\int_{\Sigma} h^{2}(s)\left|\frac{\partial w_{1, \nu}}{\partial y_{2}}\right|^{2} \mathrm{~d} \Sigma \leq C_{6}\left(\left\|P_{\mu, s} w_{1, \nu}\right\|_{L^{2}(\mathcal{Q})}^{2}+\|\mathbf{v}\|_{\left(H^{1}(\mathcal{Q})\right)^{2}}^{2}\right)
$$


Finally, by (7.6), (7.7) combined with (5.16), we obtain

$$
\left\|\left(\frac{\partial w_{2, \nu}}{\partial y_{2}}, w_{2, \nu}\right)\right\|_{X}^{2} \leq C_{7}\left(\left\|P_{\mu, s} w_{1, \nu}\right\|_{L^{2}(\mathcal{Q})}^{2}+\|\mathbf{v}\|_{\left(H^{1}(\mathcal{Q})\right)^{2}}^{2}+\|h(s) \mathbf{g}\|_{\left(L^{2}(\Sigma)\right)^{2}}^{2}\right) .
$$

Since (7.6)-(7.8), (5.31) and (5.32), we obtain (5.15).

Case B. Assume that $s^{*}=0$ and

$$
\mu\left(y^{*}\right) \varphi_{y_{2}}\left(y^{*}\right) \leq \frac{\left|\mu\left(y^{*}\right) \varphi_{y_{1}}\left(y^{*}\right) \xi_{1}^{*}-\varphi_{y_{0}}\left(y^{*}\right) \xi_{0}^{*}\right|}{\sqrt{\frac{\lambda+\mu}{\mu}\left(y^{*}\right)}\left|\xi_{1}^{*}\right|} .
$$

Then $\lim _{\zeta \rightarrow \zeta^{*}} \operatorname{Im} r_{\mu}\left(y^{*}, \zeta\right) /|s| \neq 0$. Since $s^{*}=0$, we note that $\operatorname{Re} r_{\mu}\left(y^{*}, \zeta^{*}\right)>0$. Set $I=\operatorname{sign} \lim _{\zeta \rightarrow \zeta^{*}}$ $\operatorname{Im} r_{\mu}\left(y^{*}, \zeta\right) /|s|$. Then we have

$$
\Gamma_{\mu}^{+}\left(y^{*}, \zeta^{*}\right)=I \sqrt{\operatorname{Re} r_{\mu}\left(y^{*}, \zeta^{*}\right)} .
$$

Therefore for some $C_{8}>0$ we have

$$
-\Gamma_{\mu}^{+}\left(y^{*}, \zeta^{*}\right)\left(\mu \varphi_{y_{1}}\left(y^{*}\right) \xi_{1}^{*}-\varphi_{y_{0}}\left(y^{*}\right) \xi_{0}^{*}\right)>C_{8}
$$

Taking the parameters $\delta>0$ and $\delta_{1}>0$ sufficiently small, we obtain

$$
-\operatorname{Re} \Gamma_{\mu}^{+}(y, \zeta)\left(\mu \varphi_{y_{1}}(y) \xi_{1}-\varphi_{y_{0}}(y) \xi_{0}\right)>0, \quad \forall(y, \zeta) \in B_{\delta} \times \mathcal{O}\left(\delta_{1}\right) .
$$

Let us consider estimate (6.1). Let us recall that $J_{1}, J_{2}, J_{3}$ are defined in (6.2). We have

$$
\begin{aligned}
J_{2}= & \operatorname{Re} \int_{\Sigma} 2|s| \mu \frac{\partial w_{1, \nu}}{\partial y_{2}} \overline{\left(\mu \frac{\partial w_{1, \nu}}{\partial y_{1}} \varphi_{y_{1}}\left(y^{*}\right)-\frac{\partial w_{1, \nu}}{\partial y_{0}} \varphi_{y_{0}}\left(y^{*}\right)\right)} \mathrm{d} \Sigma \\
= & \operatorname{Re} \int_{\Sigma} 2\left|D_{\sigma}\right| \mu i \Gamma_{\mu}^{+}\left(y, D^{\prime}\right) v_{1, \nu} \overline{\left(\mu \frac{\partial v_{1, \nu}}{\partial y_{1}} \varphi_{y_{1}}\left(y^{*}\right)-\frac{\partial v_{1, \nu}}{\partial y_{0}} \varphi_{y_{0}}\left(y^{*}\right)\right)} \mathrm{d} \Sigma \\
& +\operatorname{Re} \int_{\Sigma} 2\left|D_{\sigma}\right| \mu i V_{\mu}^{+}(\cdot, 0) \overline{\left(\mu \frac{\partial v_{1, \nu}}{\partial y_{1}} \varphi_{y_{1}}\left(y^{*}\right)-\frac{\partial v_{1, \nu}}{\partial y_{0}} \varphi_{y_{0}}\left(y^{*}\right)\right)} \mathrm{d} \Sigma \\
= & \operatorname{Re} \int_{\Sigma} 2 \mu\left(D_{y_{1}} \varphi_{y_{1}}\left(y^{*}\right)-D_{\left.y_{0} \varphi_{y_{0}}\left(y^{*}\right)\right) \Gamma_{\mu}^{+}\left(y, D^{\prime}\right)\left|D_{\sigma}\right|^{\frac{1}{2}} \widehat{v}_{1, \nu}\left|D_{\sigma}\right|^{\frac{1}{2} \widehat{v}_{1, \nu}}} \mathrm{d} \Sigma\right. \\
& +\operatorname{Re} \int_{\Sigma} 2\left|D_{\sigma}\right| \mu i V_{\mu}^{+}(\cdot, 0) \overline{\left(\mu \frac{\partial v_{1, \nu}}{\partial y_{1}} \varphi_{y_{1}}\left(y^{*}\right)-\frac{\partial v_{1, \nu}}{\partial y_{0}} \varphi_{y_{0}}\left(y^{*}\right)\right)} \mathrm{d} \Sigma .
\end{aligned}
$$

By (7.10) we obtain from Gårding's inequality that the first integral in the right hand side of (7.11) is negative. Consider two cases. First let

$$
\varphi_{y_{1}}\left(y^{*}\right) \xi_{1}^{*} \Gamma_{\mu}^{+}\left(y^{*}, \zeta^{*}\right)>0
$$

This inequality and (7.10) yield $\left|\xi_{0}^{*} \varphi_{y_{0}}\left(y^{*}\right)\right|>\left|\xi_{1}^{*} \mu\left(y^{*}\right) \varphi_{y_{1}}\left(y^{*}\right)\right|$. If $\xi_{0}^{*} \varphi_{y_{0}}\left(y^{*}\right)>0$ then $\Gamma_{\mu}^{+}\left(y^{*}, \zeta^{*}\right)=\left|\sqrt{r_{\mu}(\gamma)}\right|$

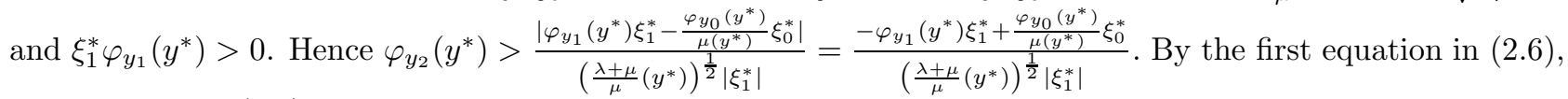
this contradicts (7.9).

If $\xi_{0} \varphi_{y_{0}}\left(y^{*}\right)<0$ then $\Gamma_{\mu}^{+}\left(y^{*}, \zeta^{*}\right)=-\left|\sqrt{r_{\mu}(\gamma)}\right|$ and $\xi_{1}^{*} \varphi_{y_{1}}\left(y^{*}\right)<0$. Therefore $\varphi_{y_{2}}\left(y^{*}\right)>\frac{\left|\varphi_{y_{1}}\left(y^{*}\right) \xi_{1}^{*}-\frac{\varphi_{y_{0}}\left(y^{*}\right)}{\mu\left(y^{*}\right)} \xi_{0}^{*}\right|}{\left(\frac{\lambda+\mu}{\mu}\left(y^{*}\right)\right)^{\frac{1}{2}}\left|\xi_{1}^{*}\right|}=$ $\frac{\varphi_{y_{1}}\left(y^{*}\right) \xi_{1}^{*}-\frac{\varphi_{y_{0}}\left(y^{*}\right)}{\mu\left(y^{*}\right)} \xi_{0}^{*}}{\left(\frac{\lambda+\mu}{\mu}\left(y^{*}\right)\right)^{\frac{1}{2}}\left|\xi_{1}^{*}\right|}$. By (2.6) this again contradicts (7.9). 
In the second case one have to consider $\varphi_{y_{1}}\left(y^{*}\right) \xi_{1}^{*} \Gamma_{\mu}^{+}\left(y^{*}, \zeta^{*}\right)<0$. By Gårding's inequality we have

$$
\operatorname{Re} \int_{\Sigma} 2\left|D_{\sigma}\right| \mu i \Gamma_{\mu}^{+}\left(y, D^{\prime}\right) v_{1, \nu} \overline{\mu\left(y^{*}\right) \varphi_{y_{1}}\left(y^{*}\right) \frac{\partial v_{1, \nu}}{\partial y_{1}}} \mathrm{~d} \Sigma<0 .
$$

This inequality and the fact that the second integral in the right hand side of $J_{2}$ is negative, imply that

$$
-\operatorname{Re} \int_{\Sigma} 2\left|D_{\sigma}\right| \mu i \Gamma_{\mu}^{+}\left(y, D^{\prime}\right) v_{1, \nu} \overline{\left((\lambda+2 \mu) \frac{\partial v_{1, \nu}}{\partial y_{1}} \varphi_{y_{1}}\left(y^{*}\right)-\frac{\partial v_{1, \nu}}{\partial y_{0}} \varphi_{y_{0}}\left(y^{*}\right)\right)} \mathrm{d} \Sigma>0 .
$$

Note that

$$
\begin{aligned}
& \Xi_{\lambda+2 \mu}^{(1)}=\int_{\Sigma}\left(|s|(\lambda+2 \mu)^{2} \varphi_{y_{2}}\left(y^{*}\right)\left|\frac{\partial w_{2, \nu}}{\partial y_{2}}\right|^{2}+|s|^{3}(\lambda+2 \mu)^{2} \varphi_{y_{2}}^{3}\left(y^{*}\right)\left|w_{2, \nu}\right|^{2}\right) \mathrm{d} \Sigma \\
& +\operatorname{Re} \int_{\Sigma} 2|s|(\lambda+2 \mu) \frac{\partial w_{2, \nu}}{\partial y_{2}} \overline{\left((\lambda+2 \mu) \varphi_{y_{1}}\left(y^{*}\right) \frac{\partial w_{2, \nu}}{\partial y_{1}}-\varphi_{y_{0}}\left(y^{*}\right) \frac{\partial w_{2, \nu}}{\partial y_{0}}\right)} \mathrm{d} \Sigma \\
& +\int_{\Sigma}|s|(\lambda+2 \mu) \varphi_{y_{2}}\left(y^{*}\right)\left(\xi_{0}^{2}-(\lambda+2 \mu) \xi_{1}^{2}-s^{2} \varphi_{y_{0}}^{2}\left(y^{*}\right)+s^{2}(\lambda+2 \mu) \varphi_{y_{1}}^{2}\left(y^{*}\right)\right)\left|\widehat{v}_{2, \nu}\right|^{2} \mathrm{~d} \Sigma \\
& =\widetilde{J}_{1}+\widetilde{J}_{2}+\widetilde{J}_{3} .
\end{aligned}
$$

Using equalities (5.14) we can transform $\widetilde{J}_{2}$ as

$$
\widetilde{J}_{2}=-\operatorname{Re} \int_{\Sigma} 2|s| \frac{\mu}{\lambda+2 \mu} \frac{\partial w_{1, \nu}}{\partial y_{2}} \overline{\left((\lambda+2 \mu) \varphi_{y_{1}}\left(y^{*}\right) \frac{\partial w_{1, \nu}}{\partial y_{1}}-\varphi_{y_{0}}\left(y^{*}\right) \frac{\partial w_{1, \nu}}{\partial y_{0}}\right)} \mathrm{d} \Sigma+I
$$

where

Then by (7.12) there exists $C_{10}>0$ such that

$$
|I| \leq \epsilon(\delta)\left\|\left(\frac{\partial \mathbf{w}_{\nu}}{\partial y_{2}}, \mathbf{w}_{\nu}\right)\right\|_{X}^{2}+C_{9}\|h(s) \mathbf{g}\|_{\left(L^{2}(\Sigma)\right)^{2}}^{2} .
$$

$$
\widetilde{J}_{2}>C_{10} \int_{\Sigma}\left(|s|\left|\frac{\partial w_{1, \nu}}{\partial y_{1}}\right|^{2}+|s|^{3}\left|w_{1, \nu}\right|^{2}\right) \mathrm{d} \Sigma .
$$

Since $r_{\lambda+2 \mu}(\gamma)=0$, we have

This inequality and (7.14) imply

$$
\left|\widetilde{J}_{3}\right| \leq C_{11}^{\prime} \delta_{1}\left\|\left(\frac{\partial w_{2, \nu}}{\partial y_{2}}, w_{2, \nu}\right)\right\|_{X}^{2}
$$

$$
\begin{aligned}
\Xi_{\lambda+2 \mu}^{(1)} \geq C_{11} \int_{\Sigma}\left(|s|\left|\frac{\partial w_{2, \nu}}{\partial y_{2}}\right|^{2}+|s|^{3}\left|w_{2, \nu}\right|^{2}+|s|\left|\frac{\partial w_{1, \nu}}{\partial y_{1}}\right|^{2}\right. & \left.+|s|^{3}\left|w_{1, \nu}\right|^{2}\right) \mathrm{d} \Sigma \\
& -\epsilon(\delta)\left\|\left(\frac{\partial \mathbf{w}_{\nu}}{\partial y_{2}}, \mathbf{w}_{\nu}\right)\right\|_{X}^{2}+C_{9}\|h(s) \mathbf{g}\|_{\left(L^{2}(\Sigma)\right)^{2}}^{2} .
\end{aligned}
$$

Now we will estimate $J_{3}$. By (5.18) and (5.19), there exists a constant $C_{12}^{\prime}>0$ such that

$$
\begin{aligned}
& \left|\xi_{0}^{2}-s^{2} \varphi_{y_{0}}^{2}\left(y^{*}\right)-(\lambda+2 \mu) \xi_{1}^{2}+(\lambda+2 \mu) s^{2} \varphi_{y_{1}}^{2}\left(y^{*}\right)\right| \\
\leq & C_{12}^{\prime} \delta_{1}\left(\left|\xi_{0}\right|^{2}+\left|\xi_{1}\right|^{2}+s^{2}\right), \quad \forall \zeta \in \mathcal{O}\left(\delta_{1}\right) .
\end{aligned}
$$


Using this inequality we obtain

$$
\begin{aligned}
& \xi_{0}^{2}-\mu \xi_{1}^{2}-s^{2} \varphi_{y_{0}}^{2}\left(y^{*}\right)+s^{2} \mu \varphi_{y_{1}}^{2}\left(y^{*}\right) \\
= & (\lambda+\mu)\left(\xi_{1}^{2}-s^{2} \varphi_{y_{1}}^{2}\left(y^{*}\right)\right)+\left(\xi_{0}^{2}-(\lambda+2 \mu) \xi_{1}^{2}-s^{2} \varphi_{y_{0}}^{2}\left(y^{*}\right)+s^{2}(\lambda+2 \mu) \varphi_{y_{1}}^{2}\left(y^{*}\right)\right) \\
\geq & (\lambda+\mu)\left(\xi_{1}^{2}-s^{2} \varphi_{y_{1}}^{2}\left(y^{*}\right)\right)-C_{12} \delta_{1}\left(\left|\xi_{0}\right|^{2}+\left|\xi_{1}\right|^{2}+s^{2}\right) .
\end{aligned}
$$

Therefore, for all sufficiently small $\delta_{1}$, there exists $C_{13}>0$ such that

$$
\xi_{0}^{2}-\mu \xi_{1}^{2}-s^{2} \varphi_{y_{0}}^{2}\left(y^{*}\right)+s^{2} \mu \varphi_{y_{1}}^{2}\left(y^{*}\right) \geq C_{13}\left(\left|\xi_{0}\right|^{2}+\left|\xi_{1}\right|^{2}+s^{2}\right)
$$

By (7.17), we see that $J_{3} \geq 0$. Therefore by (7.15) and (6.1), there exist constants $C_{13}^{\prime}>0, C_{14}>0$ such that

$$
\begin{array}{r}
\Xi_{\mu}^{(1)}+C_{13}^{\prime} \Xi_{\lambda+2 \mu}^{(1)} \geq C_{14}\left\|\left(\frac{\partial w_{1, \nu}}{\partial y_{2}}, w_{1, \nu}\right)\right\|_{X}^{2}-C_{10}\left(\delta, \delta_{1}\right)\left(\left\|P_{\mu, s} w_{1, \nu}\right\|_{L^{2}(\mathcal{Q})}^{2}+\|\mathbf{v}\|_{\left(H^{1}(\mathcal{Q})\right)^{2}}^{2}\right) \\
-\epsilon(\delta)\left\|\left(\frac{\partial \mathbf{w}_{\nu}}{\partial y_{2}}, \mathbf{w}_{\nu}\right)\right\|_{X}^{2}+C_{9}\|h(s) \mathbf{g}\|_{\left(L^{2}(\Sigma)\right)^{2}}^{2}
\end{array}
$$

This inequality, (5.16) and (7.4) with the sign + , imply

$$
\begin{gathered}
\Xi_{\mu}^{(1)} \geq C_{15}\left\|\left(\frac{\partial \mathbf{w}_{\nu}}{\partial y_{2}}, \mathbf{w}_{\nu}\right)\right\|_{X}^{2} \\
-C_{16}\left(\delta, \delta_{1}\right)\left(\left\|P_{\mu, s} w_{1, \nu}\right\|_{L^{2}(\mathcal{Q})}^{2}+\|h(s) \mathbf{g}\|_{\left(L^{2}(\Sigma)\right)^{2}}^{2}+\|\mathbf{v}\|_{\left(H^{1}(\mathcal{Q})\right)^{2}}^{2}\right) .
\end{gathered}
$$

By (7.18), (5.31) and (5.32), we obtain (5.15).

Case C. Assume that $s^{*} \neq 0$. If $\delta_{1}>0$ is small enough, then there exists a constant $C_{17}>0$ such that

$$
\left|\xi_{0} \varphi_{y_{1}}\left(y^{*}\right)-(\lambda+2 \mu) \xi_{1} \varphi_{y_{1}}\left(y^{*}\right)\right|^{2} \leq \delta_{1}^{2} C_{17}\left(\left|\xi_{1}\right|^{2}+s^{2}\right)
$$

By (5.31), there exists $C_{18}>0$ such that

$$
\begin{aligned}
\Xi_{\lambda+2 \mu}^{(1)}+C_{18}\left(\left\|h(s) w_{2, \nu}\right\|_{H^{1}(\mathcal{Q})}^{2}+\left\|h^{3}(s) w_{2, \nu}\right\|_{L^{2}(\mathcal{Q})}^{2}\right) & \\
& \leq C_{18}\left(\left\|\mathbf{P}_{\lambda+2 \mu} v_{2}\right\|_{L^{2}(\mathcal{Q})}^{2}+\|\mathbf{v}\|_{\left(H^{1}(\mathcal{Q})\right)^{2}}^{2}\right)+\epsilon\left\|\left(\frac{\partial w_{2, \nu}}{\partial y_{2}}, w_{2, \nu}\right)\right\|_{X}^{2}
\end{aligned}
$$

By (7.16) and (7.19), we have

$$
\left|\widetilde{J}_{2}+\widetilde{J}_{3}\right| \leq C_{19} \delta_{1}\left\|\left(\frac{\partial w_{2, \nu}}{\partial y_{2}}, w_{2, \nu}\right)\right\|_{X}^{2} .
$$

By (7.21) we obtain from (7.13) that there exists a constant $C_{20}>0$ such that

$$
\begin{aligned}
& \Xi_{\lambda+2 \mu}^{(1)} \geq-\epsilon\left\|\left(\frac{\partial w_{2, \nu}}{\partial y_{2}}, w_{2, \nu}\right)\right\|_{X}^{2} \\
& \quad+C_{20} \int_{\Sigma}\left(h^{2}(s)(\lambda+2 \mu)^{2} \varphi_{y_{2}}\left(y^{*}\right)\left|\frac{\partial w_{2, \nu}}{\partial y_{2}}\right|^{2}+h^{6}(s)(\lambda+2 \mu)^{2} \varphi_{y_{2}}^{3}\left(y^{*}\right)\left|w_{2, \nu}\right|^{2}\right) \mathrm{d} \Sigma
\end{aligned}
$$


From (5.16), we easily obtain

$$
\left\|h(s)\left(\frac{\partial w_{2, \nu}}{\partial y_{2}}-|s| \varphi_{y_{2}}\left(y^{*}\right) w_{2, \nu}+g_{2, \nu}\right)\right\|_{L^{2}(\Sigma)}^{2}=\frac{\mu^{2}}{(\lambda+2 \mu)^{2}}\left(\left\|h(s) \frac{\partial w_{1, \nu}}{\partial y_{1}}\right\|_{L^{2}(\Sigma)}^{2}+\varphi_{y_{1}}^{2}\left(y^{*}\right)\left\|h(s)|s| w_{1, \nu}\right\|_{L^{2}(\Sigma)}^{2}\right) .
$$

Hence (7.22) and this equality imply

$$
\Xi_{\lambda+2 \mu}^{(1)} \geq C_{21} \int_{\Sigma}\left(h^{2}(s)\left(\left|\frac{\partial w_{2, \nu}}{\partial y_{2}}\right|^{2}+\left|\frac{\partial w_{1, \nu}}{\partial y_{1}}\right|^{2}\right)+h^{6}(s)\left|w_{2, \nu}\right|^{2}\right) \mathrm{d} \Sigma-\epsilon\left\|\left(\frac{\partial w_{2, \nu}}{\partial y_{2}}, w_{2, \nu}\right)\right\|_{X}^{2}-C_{22}\|h(s) \mathbf{g}\|_{\left(L^{2}(\Sigma)\right)^{2}}^{2}
$$

Now we claim that inequality (7.2) holds true for all sufficiently small $\delta_{1}$. First we may assume that for all $\zeta \in \mathcal{O}\left(\delta_{1}\right)$ we have $s^{2} \leq C_{23}\left(\xi_{0}^{2}+\xi_{1}^{2}\right)$. In fact, if the last inequality is not true, then $\zeta^{*} \in \mathcal{M}$ and the case was treated in the previous section. Suppose that (7.2) is not true. In that case $\xi_{1}^{*}=0$ and $\xi_{0}^{*} \neq 0, s^{*} \neq 0$. Therefore $\varphi_{y_{0}}\left(y^{*}\right)=0$ by (5.18). However, this implies $\left(\xi_{0}^{*}\right)^{2}+\left(\lambda\left(y^{*}\right)+2 \mu\left(y^{*}\right)\right) \varphi_{y_{1}}^{2}\left(y^{*}\right)\left(s^{*}\right)^{2}=0$. Hence we arrived at a contradiction and the verification of $(7.2)$ is complete.

Inequalities (7.2) and (7.23) imply that there exists a constant $C_{24}>0$ such that

$$
\begin{aligned}
\Xi_{\lambda+2 \mu}^{(1)} \geq & C_{24} \int_{\Sigma}\left(h^{2}(s)\left(\left|\frac{\partial w_{2, \nu}}{\partial y_{2}}\right|^{2}+\left|\frac{\partial w_{1, \nu}}{\partial y_{1}}\right|^{2}+\left|\frac{\partial w_{1, \nu}}{\partial y_{0}}\right|^{2}\right)+h^{6}(s)\left|\mathbf{w}_{\nu}\right|^{2}\right) \mathrm{d} \Sigma \\
& -\epsilon\left\|\left(\frac{\partial w_{2, \nu}}{\partial y_{2}}, w_{2, \nu}\right)\right\|_{X}^{2}-C_{22}\|h(s) \mathbf{g}\|_{\left(L^{2}(\Sigma)\right)^{2}}^{2}
\end{aligned}
$$

By inequality $(7.4)$ for $V_{\mu}^{+}(\cdot, 0)$, we obtain the estimate

$$
\begin{gathered}
\left\|h(s) \frac{\partial w_{1, \nu}}{\partial y_{2}}\right\|_{L^{2}(\Sigma)}^{2} \leq C_{25}\left\{\int_{\Sigma}\left(h^{2}(s)\left(\left|\frac{\partial w_{1, \nu}}{\partial y_{1}}\right|^{2}+\left|\frac{\partial w_{1, \nu}}{\partial y_{0}}\right|^{2}\right)+h^{6}(s)\left|w_{1, \nu}\right|^{2}\right) \mathrm{d} \Sigma\right. \\
\left.+\left\|\mathbf{P}_{\mu} v_{1, \nu}\right\|_{L^{2}(\mathcal{Q})}^{2}+\|\mathbf{v}\|_{\left(H^{1}(\mathcal{Q})\right)^{2}}^{2}\right\}
\end{gathered}
$$

Inequalities (7.24) and (7.25) imply that there exists a constant $C_{26}>0$ such that

$$
\Xi_{\lambda+2 \mu}^{(1)} \geq C_{26}\left\|\left(\frac{\partial \mathbf{w}_{\nu}}{\partial y_{2}}, \mathbf{w}_{\nu}\right)\right\|_{X}^{2}-C_{27}\left(\delta, \delta_{1}\right)\left(\left\|P_{\mu, s} w_{1, \nu}\right\|_{L^{2}(\mathcal{Q})}^{2}+\|h(s) \mathbf{g}\|_{\left(L^{2}(\Sigma)\right)^{2}}^{2}+\|\mathbf{v}\|_{\left(H^{1}(\mathcal{Q})\right)^{2}}^{2}\right)
$$

By (7.26), (5.31) and (5.32), we obtain (5.15). The proof of Lemma 7.1 is finished.

$$
\text { 8. ThE } \operatorname{CASE} r_{\mu}(\gamma) \neq 0 \text { AND } r_{\lambda+2 \mu}(\gamma) \neq 0
$$

In this section, we will prove

Lemma 8.1. Let $\gamma=\left(y^{*}, \zeta^{*}\right) \in \Sigma \times \mathbb{S}^{2}$ be a point such that

$$
\left|r_{\mu}\left(y^{*}, \zeta^{*}\right)\right| \neq 0 \quad \text { and } \quad\left|r_{\lambda+2 \mu}\left(y^{*}, \zeta^{*}\right)\right| \neq 0
$$

If $\operatorname{supp} \chi_{\nu} \subset \mathcal{O}\left(\delta_{1}\right)$ and $\delta_{1}>0$ is sufficiently small, then estimate (5.15) holds true. 
Proof. Thanks to (8.1) and Proposition 5.3, decomposition (5.23) holds true for $\beta=\mu$ and $\beta=\lambda+2 \mu$. Therefore we have

$$
\begin{aligned}
\left.\left(D_{y_{2}}-\Gamma_{\mu}^{+}\left(y, D^{\prime}\right)\right) v_{1, \nu}\right|_{y_{2}=0} & =V_{\mu}^{+}(\cdot, 0), \\
\left.\left(D_{y_{2}}-\Gamma_{\lambda+2 \mu}^{+}\left(y, D^{\prime}\right)\right) v_{2, \nu}\right|_{y_{2}=0} & =V_{\lambda+2 \mu}^{+}(\cdot, 0) .
\end{aligned}
$$

By Proposition 5.4, we have an a priori estimate

$$
\left\|h\left(D_{\sigma}\right) V_{\mu}^{+}(\cdot, 0)\right\|_{L^{2}(\Sigma)}^{2}+\left\|h\left(D_{\sigma}\right) V_{\lambda+2 \mu}^{+}(\cdot, 0)\right\|_{L^{2}(\Sigma)}^{2} \leq C_{1}\left(\left\|\mathbf{P}_{\lambda+2 \mu} v_{2}\right\|_{L^{2}(\mathcal{Q})}^{2}+\left\|\mathbf{P}_{\mu} v_{1}\right\|_{L^{2}(\mathcal{Q})}^{2}+\|\mathbf{v}\|_{\left(H^{1}(\mathcal{Q})\right)^{2}}^{2}\right)
$$

Denote

$$
\tilde{\alpha}_{\mu}^{+}\left(y^{\prime}, D\right)=\alpha_{\mu}^{+}\left(y^{\prime}, D\right)+i\left|D_{\sigma}\right|\left(\varphi_{y_{2}}-\varphi_{y_{2}}\left(y^{*}\right)\right) .
$$

Using (5.16), we may rewrite (8.2) and (8.3) as

$$
\begin{gathered}
\frac{\lambda+2 \mu}{\mu}\left(\frac{\partial v_{2, \nu}}{\partial y_{1}}-\left|D_{\sigma}\right| \varphi_{y_{1}}\left(y^{*}\right) v_{2, \nu}\right)-i \tilde{\alpha}_{\mu}^{+}\left(y, D^{\prime}\right) v_{1, \nu}=i V_{\mu}^{+}(\cdot, 0)-i \mathcal{F}_{\sigma}^{-1} g_{1, \nu}, \\
\frac{\mu}{\lambda+2 \mu}\left(-\frac{\partial v_{1, \nu}}{\partial y_{1}}+\left|D_{\sigma}\right| \varphi_{y_{1}}\left(y^{*}\right) v_{1, \nu}\right)-i \tilde{\alpha}_{\lambda+2 \mu}^{+}\left(y, D^{\prime}\right) v_{2, \nu}=i V_{\lambda+2 \mu}^{+}(\cdot, 0)-i \mathcal{F}_{\sigma}^{-1} g_{2, \nu} .
\end{gathered}
$$

Let $\mathbf{B}\left(y, D^{\prime}\right)$ be the matrix pseudo-differential operator with the symbol

$$
\mathbf{B}(y, \zeta)=\left(\begin{array}{cc}
-i \tilde{\alpha}_{\mu}^{+}(y, \zeta) & \frac{\lambda+2 \mu}{\mu}\left(i \xi_{1}-|s| \varphi_{y_{1}}(y)\right) \\
\frac{\mu}{\lambda+2 \mu}\left(-i \xi_{1}+|s| \varphi_{y_{1}}(y)\right) & -i \tilde{\alpha}_{\lambda+2 \mu}^{+}(y, \zeta)
\end{array}\right)
$$

By (5.19) and (5.20), we see: if $\operatorname{det} \mathbf{B}\left(y^{*}, \zeta^{*}\right)=0$, then either $\xi_{0}^{*}+i s^{*} \varphi_{y_{0}}\left(y^{*}\right)=0$ or

$$
\zeta^{*} \in\left\{\zeta \in \mathbb{R}^{3} ;\left(\xi_{1}+i|s| \varphi_{y_{1}}\left(y^{*}\right)\right)^{2}=\frac{\left(\xi_{0}+i|s| \varphi_{y_{0}}\left(y^{*}\right)\right)^{2}}{(\lambda+3 \mu)\left(y^{*}\right)}\right\} .
$$

In this case of $(8.7)$, we have $\varphi_{y_{0}}\left(y^{*}\right)=\varphi_{y_{1}}\left(y^{*}\right)=\xi_{0}^{*}=\xi_{1}^{*}=0, s^{*}=1$.

Now we consider two cases

Case A. $\operatorname{det} \mathbf{B}(\gamma) \neq 0$.

In this case, there exists a parametrix of the operator $\mathbf{B}\left(y, D^{\prime}\right)$, which we denote by $\mathbf{B}^{-1}\left(y, D^{\prime}\right)$, such that

$$
\left(v_{1, \nu}, v_{2, \nu}\right)=\mathbf{B}^{-1}\left(y, D^{\prime}\right)\left(V_{\mu}^{+}(\cdot, 0)-\mathcal{F}_{\sigma}^{-1} g_{1, \nu}, V_{\lambda+2 \mu}^{+}(\cdot, 0)-\mathcal{F}_{\sigma}^{-1} g_{2, \nu}\right)^{T}+K\left(v_{1, \nu}, v_{2, \nu}\right),
$$

where $K:\left(L^{2}(\mathcal{Q})\right)^{2} \rightarrow\left(H^{1}(\mathcal{Q})\right)^{2}$. By $(8.4)$ and $(8.8)$,

$$
\left|\Xi_{\mu}\right|+\left|\Xi_{\lambda+2 \mu}\right| \leq C_{2}\left(\left\|\mathbf{P}_{\mu} v_{1}\right\|_{L^{2}(\mathcal{Q})}^{2}+\left\|\mathbf{P}_{\lambda+2 \mu} v_{2}\right\|_{L^{2}(\mathcal{Q})}^{2}+\|h(s) \mathbf{g}\|_{\left(L^{2}(\Sigma)\right)^{2}}^{2}+\|\mathbf{v}\|_{\left(H^{1}(\mathcal{Q})\right)^{2}}^{2}\right) .
$$

(Here and henceforth, for simplicity, we do not distinguish $\mathbf{a}^{T}$ from a vector a.) By (8.9), (5.30) and (5.31), we obtain (5.15).

Case B. $\operatorname{det} \mathbf{B}(\gamma)=0$.

We claim that this situation is possible in the two cases:

$$
\begin{aligned}
& \text { (i) } \varphi_{y_{0}}\left(y^{*}\right)=\varphi_{y_{1}}\left(y^{*}\right)=\xi_{0}^{*}=\xi_{1}^{*}=0, \quad s^{*}=1 \\
& \text { (ii) } \xi_{0}^{*}=0, \quad s^{*} \varphi_{y_{0}}\left(y^{*}\right)=0 .
\end{aligned}
$$


The first subcase was treated in Section 6. Let us consider the second subcase (8.10). Next we may assume that

$$
\zeta^{*} \in \widetilde{\mathcal{M}}
$$

Otherwise, $\zeta^{*} \in \mathcal{M}$, so that the case was treated in Section 6 . Next we may assume that

$$
\operatorname{Im} \Gamma_{\mu}^{+}(\gamma)=\operatorname{Im} \Gamma_{\lambda+2 \mu}^{+}(\gamma) \geq 0
$$

Really if

$$
\operatorname{Im} \Gamma_{\mu}^{+}(\gamma)=\operatorname{Im} \Gamma_{\lambda+2 \mu}^{+}(\gamma)<0
$$

then the situation is simple since we have the decomposition

$$
\mathbf{P}_{\beta} v_{j(\beta), \nu}=\beta|G|^{2}\left(D_{y_{2}}-\Gamma_{\beta}^{\mp}\left(y, D^{\prime}\right)\right) V_{\beta}^{ \pm}+T_{\mu}^{ \pm} v_{j(\beta), \nu}
$$

where $T_{\beta}^{ \pm} \in \mathcal{L}\left(H^{1}(\mathcal{Q}), L^{2}(\mathcal{Q})\right), \beta \in\{\mu, \lambda+2 \mu\}, j(\beta)=1$ for $\beta=\mu$ and $j(\beta)=2$ for $\beta=\lambda+2 \mu$. This decomposition, (8.12) and Proposition 5.3 imply

$$
\left\|\left.h\left(D_{\sigma}\right)\left(D_{y_{2}}-\Gamma_{\beta}^{ \pm}\left(y, D^{\prime}\right)\right) v_{j(\beta), \nu}\right|_{y_{2}=0}\right\|_{L^{2}(\Sigma)} \leq C_{3}\left(\left\|\mathbf{P}_{\beta} v_{j(\beta), \nu}\right\|_{L^{2}(\mathcal{Q})}+\|\mathbf{v}\|_{\left(H^{2}(\mathcal{Q})\right)^{2}}\right)
$$

Obviously

$$
-V_{\beta}^{+}(\cdot, 0)+V_{\beta}^{-}(\cdot, 0)=\left(\alpha_{\beta}^{+}\left(y, D^{\prime}\right)-\alpha_{\beta}^{-}\left(y, D^{\prime}\right)\right) v_{1, \nu} \quad \text { on } \Sigma .
$$

Since $\alpha_{\mu}^{+}\left(y^{*}, \zeta^{*}\right)-\alpha_{\mu}^{-}\left(y^{*}, \zeta^{*}\right)=2 \sqrt{r_{\mu}\left(y^{*}, \zeta^{*}\right)} \neq 0$, we have

$$
\left\|\left(\frac{\partial \mathbf{w}_{\nu}}{\partial y_{2}}, \mathbf{w}_{\nu}\right)\right\|_{X}^{2} \leq C_{4}\left(\left\|P_{\lambda+2 \mu, s} w_{2, \nu}\right\|_{L^{2}(\mathcal{Q})}^{2}+\left\|P_{\mu, s} w_{1, \nu}\right\|_{L^{2}(\mathcal{Q})}^{2}+\|\mathbf{v}\|_{\left(H^{1}(\mathcal{Q})\right)^{2}}^{2}\right)
$$

by (8.13) and Gårding's inequality.

By (8.14), (5.30) and (5.31), we obtain (5.15) under Condition (8.12).

In order to treat (8.10) under (8.11), we will use Calderon's method. First we introduce the new variables $U=\left(U_{1}, U_{2}, U_{3}, U_{4}\right)$ with four components, where

$$
\left(U_{1}, U_{2}\right)=\Lambda\left(D^{\prime}\right) \mathcal{F}_{\sigma}^{-1} \mathcal{U}, \quad\left(U_{3}, U_{4}\right)=\left(D_{2}+i\left|D_{\sigma}\right| \varphi_{y_{2}}\right) \mathcal{F}_{\sigma}^{-1} \mathcal{U}
$$

and $\Lambda$ is the pseudo-differential operator with the symbol $\left(s^{2}+\xi_{1}^{2}+\xi_{0}^{2}+1\right)^{\frac{1}{2}}$. In the new notations, problem (5.1) and (5.2) can be written in the form

$$
D_{y_{2}} U=M\left(y, D^{\prime}\right) U+F \quad \text { in } \mathbb{R}^{3} \times[0,1],\left.\quad\left(U_{1}, U_{2}\right)(y)\right|_{y_{2}=0}=0,
$$

where $F=\left(0, \mathbb{P}_{\sigma} \mathcal{F}_{\sigma}^{-1} \mathcal{U}\right)$. Here $M\left(y, D^{\prime}\right)$ is the matrix pseudo-differential operator whose principal symbol $M_{1}(y, \zeta)$ is given by

$$
M_{1}(y, \zeta)=\left(\begin{array}{cc}
0 & \Lambda_{1} E_{2} \\
A^{-1} M_{21} \Lambda_{1}^{-1} & A^{-1} M_{22}
\end{array}\right)-i|s| \varphi_{y_{2}} E_{4}
$$

(see [49]). Here we set $\vec{\theta}=\left(\xi_{1}+i|s| \varphi_{y_{1}}, 0\right), G\left(y_{1}\right)=\left(-\mathrm{d} \ell\left(y_{1}\right) / \mathrm{d} y_{1}, 1\right), \Lambda_{1}=|\zeta|, M_{21}\left(y, \xi^{\prime}+i|s| \nabla_{y^{\prime}} \varphi(y)\right)=$ $\left(\left(\xi_{0}+i|s| \varphi_{y_{0}}(y)\right)^{2}-\mu\left(\xi^{\prime}+i|s| \varphi_{y_{1}}(y)\right)^{2}\right) E_{2}-(\lambda+\mu)(y) \vec{\theta}^{T} \vec{\theta}, M_{22}\left(y, \xi^{\prime}\right)=-(\lambda+\mu)(y) \vec{\theta}^{T} G+G^{T} \vec{\theta}-2 \mu(\vec{\theta}, G) E_{2}$, $A=(\lambda+\mu)(y) G^{T} G+\mu(y)|G|^{2} E_{2}$. The matrix $M_{1}(\gamma)$ has only two eigenvalues $M^{ \pm}$given by (5.18)-(5.20). Moreover it is known that the Jordan form of the matrix $M_{1}(\gamma)$ has two Jordan blocks of the form

$$
M^{ \pm}=\left(\begin{array}{cc}
\Gamma_{\mu}^{ \pm}(\gamma) & 1 \\
0 & \Gamma_{\mu}^{ \pm}(\gamma)
\end{array}\right)
$$


Following [46] and using the change of variables $W=S^{-1}\left(y, D^{\prime}\right) U$ which is constructed below, we can reduce system (8.15) to the form

$$
D_{y_{2}} W=\widetilde{M}\left(y, D^{\prime}\right) W+T\left(y, D^{\prime}\right) W+\widetilde{F}
$$

where the matrix $\widetilde{M}$ has the form

$$
\widetilde{M}(y, \zeta)=\left(\begin{array}{cc}
M_{+}(y, \zeta) & 0 \\
0 & M_{-}(y, \zeta)
\end{array}\right), \quad M_{ \pm}=\left(\begin{array}{cc}
\Gamma_{\lambda+2 \mu}^{ \pm}(y, \zeta) & m_{12}^{ \pm}(y, \zeta) \\
0 & \Gamma_{\mu}^{ \pm}(y, \zeta)
\end{array}\right)
$$

the operator $T$ is in $L^{\infty}\left(0,1 ; \mathcal{L}\left(\left(H^{1}(\Sigma)\right)^{4},\left(H^{1}(\Sigma)\right)^{4}\right)\right), m_{12}^{ \pm}\left(y, D^{\prime}\right)$ are first order operators and

$$
\|\widetilde{F}\|_{L^{2}\left(\mathbb{R}^{1} ;\left(H^{1}(\Sigma)\right)^{2}\right)} \leq C_{5}\left(\left\|\mathbb{P}_{\sigma} \mathcal{F}_{\sigma}^{-1} \mathcal{U}\right\|_{\left(H^{1}(\mathcal{Q})\right)^{2}}+\left\|\mathcal{F}_{\sigma}^{-1} \mathcal{U}\right\|_{L^{2}\left(\mathbb{R}^{1} ;\left(H^{1}(\Sigma)\right)^{2}\right)}\right)
$$

Now we describe the construction of the pseudo-differential operator $S$. We take the symbol $S$ in the form $S=\left(s_{1}^{+}, s_{2}^{+}, s_{1}^{-}, s_{2}^{-}\right)$. Here

$$
s_{1}^{ \pm}=\left(\left(\vec{\theta}+\alpha_{\lambda+2 \mu}^{ \pm} G\right) \Lambda_{1}^{-1}, \alpha_{\lambda+2 \mu}^{ \pm}\left(\vec{\theta}+\alpha_{\lambda+2 \mu}^{ \pm} G\right) \Lambda_{1}^{-2}\right)
$$

are the eigenvectors of the matrix $M_{1}(y, \zeta)$ on the sphere $\zeta \in \mathbb{S}^{2}$ which corresponds to the eigenvalue $\Gamma_{\lambda+2 \mu}^{ \pm}$and the vectors $s_{2}^{ \pm}$are given by the formula

$$
s_{2}^{ \pm}=E_{ \pm} s^{ \pm}, \quad E_{ \pm}=\frac{1}{2 \pi i} \int_{C^{ \pm}}\left(z-M_{1}(y, \zeta)\right)^{-1} \mathrm{~d} z
$$

where $C^{ \pm}$are small circles centered at $\Gamma_{\mu}^{ \pm}(\gamma)$ and $s^{ \pm}$solves the equation $M_{1}(\gamma) s^{ \pm}-\Gamma_{\mu}^{ \pm}(\gamma) s^{ \pm}=s_{1}^{ \pm}(\gamma)$. Since $\zeta^{*} \in \widetilde{\mathcal{M}}$ and $\xi_{0}^{*}=0$, we have $\xi_{1}^{*} \neq 0$. Therefore the circles $C^{ \pm}$may be taken such that the disks bounded by these circles do not intersect, provided that $\delta_{1}, \delta$ are taken sufficiently small. Note that the vectors $s_{j}^{ \pm} \in C^{2}\left(B_{\delta} \times \mathcal{O}_{\delta_{1}}\right)$ are homogeneous functions of the order zero in $\left(s, \xi_{0}, \xi_{1}\right)$. Now using a standard argument (see [36], p. 241), we can estimate the last two components of $W$ as follows

$$
\left\|\left(W_{3}, W_{4}\right)\right\|_{\left(H^{\frac{3}{2}}(\Sigma)\right)^{2}} \leq C_{6}\left(\left\|\mathbb{P}_{\sigma} \mathcal{F}_{\sigma}^{-1} \mathcal{U}\right\|_{\left(H^{1}(\mathcal{Q})\right)^{2}}+\left\|\mathcal{F}_{\sigma}^{-1} \mathcal{U}\right\|_{\left(H^{2}(\mathcal{Q})\right)^{2}}\right)
$$

where the constant $C_{6}$ is independent of $N$.

Now we need to estimate the first two components of the vector function $W$ on $\Sigma$. Thanks to the zero boundary conditions for $U_{3}$ and $U_{4}$, we have

$$
S_{11}\left(y_{0}, y_{1}, 0, D^{\prime}\right)\left(W_{1}, W_{2}\right)=-S_{12}\left(y_{0}, y_{1}, 0, D^{\prime}\right)\left(W_{3}, W_{4}\right)+T_{-1}\left(y_{0}, y_{1}, 0, D^{\prime}\right) \mathcal{F}_{\sigma}^{-1} \mathcal{U}
$$

where we set

$$
S(y, \zeta)=\left(\begin{array}{ll}
S_{11}(y, \zeta) & S_{12}(y, \zeta) \\
S_{21}(y, \zeta) & S_{22}(y, \zeta)
\end{array}\right), \quad T_{-1}:\left(H^{1}(\Sigma)\right)^{2} \rightarrow\left(H^{2}(\Sigma)\right)^{2}
$$

The principal symbol of the pseudo-differential operator $S_{11}$ is the $2 \times 2$ matrix such that the first column equals the last two coordinates of the vector $s_{1}^{+}$and the second column equals the last two coordinates of the vector $s_{2}^{+}$. At the point $\gamma$, these vectors are given by the formulae

$$
\begin{gathered}
\vec{\eta}=\left(\xi_{1}^{*}+i\left|s^{*}\right| \varphi_{y_{1}}\left(y^{*}\right), i \operatorname{sign}\left(\xi_{1}^{*}\right)\left(\xi_{1}^{*}+i\left|s^{*}\right| \varphi_{y_{1}}\left(y^{*}\right)\right)\right) \\
s_{1}^{+}(\gamma)=\left(\vec{\eta}, i \frac{\operatorname{sign}\left(\xi_{1}^{*}\right)\left(\xi_{1}^{*}+i\left|s^{*}\right| \varphi_{y_{1}}\left(y^{*}\right)\right)}{\sqrt{\left(\xi_{1}^{*}\right)^{2}+\left(s^{*}\right)^{2}}} \vec{\eta}\right)
\end{gathered}
$$




$$
\begin{gathered}
\vec{\varsigma}=\frac{-1}{\sqrt{\left(\xi_{1}^{*}\right)^{2}+\left(s^{*}\right)^{2}}} \frac{\lambda+3 \mu}{2(\lambda+\mu)}\left(y^{*}\right)\left(i \operatorname{sign}\left(\xi_{1}^{*}\right), 1\right), \\
s_{2}^{+}(\gamma)=\left(\vec{\varsigma}, \frac{1}{\sqrt{\left(\xi_{1}^{*}\right)^{2}+\left(s^{*}\right)^{2}}}\left\{i \operatorname{sign}\left(\xi_{1}^{*}\right)\left(\xi_{1}^{*}+i\left|s^{*}\right| \varphi_{y_{1}}\left(y^{*}\right)\right) \vec{\varsigma}+\vec{\eta}\right\}\right) .
\end{gathered}
$$

Therefore $\operatorname{det} S_{11}(\gamma) \neq 0$. From (8.15), (8.16) and Gårding's inequality, we obtain

$$
\left\|\left(\frac{\partial \mathbf{w}_{\nu}}{\partial y_{2}}, \mathbf{w}_{\nu}\right)\right\|_{X} \leq C_{7}\left(\left\|\mathbb{P}_{\sigma} \mathcal{F}_{\sigma}^{-1} \mathcal{U}\right\|_{\left(H^{1}(\mathcal{Q})\right)^{2}}+\left\|\mathcal{F}_{\sigma}^{-1} \mathcal{U}\right\|_{\left(H^{2}(\mathcal{Q})\right)^{2}}\right),
$$

where the constant $C_{7}$ is independent of $N$. By (8.9), (5.30) and (5.31), we obtain (5.15). The proof of Lemma 8.1 is finished.

\section{Proofs of Theorems 2.2 AND 2.3}

In this section we prove Theorems 2.2 and 2.3. The proof is based on the duality argument and the scenario is described as follows. In view of the fact that observability implies controllability and vice versa, we will introduce an extremal problem, and, using Carleman estimate (2.9), we show that there exists a solution to this problem which solves the control problem for the operator $P^{*}$ and minimizes weighted $L^{2}(Q)$-norm. At the next step, we obtain an estimate of this solution in the weighted $H^{1}$-norm. This estimate implies (2.11) and (2.12).

We introduce the Banach space $\mathcal{X}=\left(H^{1}(Q)\right)^{2}$ with the norm

$$
\|\mathbf{w}\|_{\mathcal{X}}^{2}=\int_{Q}\left(|\nabla \mathbf{w}|^{2}+s^{2}|\mathbf{w}|^{2}\right) \mathrm{d} x
$$

In order to prove the theorems, we consider the following extremal problem

$$
\begin{gathered}
J\left(\mathbf{z}, \mathbf{v}_{1}, \mathbf{v}_{2}\right)=\frac{1}{2}\left\|\mathbf{z} \mathrm{e}^{-s \phi}\right\|_{\left(L^{2}(Q)\right)^{2}}^{2}+\frac{1}{2}\left\|\mathbf{v}_{1} \mathrm{e}^{-s \phi}\right\|_{\left(L^{2}\left(Q_{\omega}\right)\right)^{2}}^{2}+\frac{1}{2 s^{2}}\left\|\mathbf{v}_{2} \mathrm{e}^{-s \phi}\right\|_{\left(L^{2}\left(Q_{\omega}\right)\right)^{2}}^{2} \longrightarrow \inf , \\
P \mathbf{z}=\mathbf{u} e^{2 s \phi}+\frac{\partial \mathbf{v}_{1}}{\partial x_{0}}+\mathbf{v}_{2} \quad \text { in } Q, \\
\operatorname{supp} \mathbf{v}_{j} \subset \overline{Q_{\omega}}, \quad j=1,2,\left.\quad \mathbf{z}\right|_{(0, T) \times \partial \Omega}=0, \quad \frac{\partial \mathbf{z}}{\partial x_{0}}(0, \cdot)=\frac{\partial \mathbf{z}}{\partial x_{0}}(T, \cdot)=0 .
\end{gathered}
$$

Denote by $\left(\mathbf{z}, \mathbf{v}_{1}, \mathbf{v}_{2}\right)$ the solution to extremal problem (9.1)-(9.3).

We have

Lemma 9.1. Under the conditions of Theorem 2.2 for all $\mathbf{u} \in\left(L^{2}(Q)\right)^{2}$, there exists a unique solution $\left(\mathbf{z}, \mathbf{v}_{1}, \mathbf{v}_{2}\right) \in\left(H^{1}(Q)\right)^{2} \times\left(H^{1}\left(0, T ; L^{2}(\Omega)\right)\right)^{2} \times\left(H^{1}(Q)\right)^{2}$ to problem (9.1)-(9.3). Moreover this solution satisfies the optimality system

$$
\begin{gathered}
P \mathbf{p}+\mathbf{z} \mathrm{e}^{-2 s \phi}=0 \quad \text { in } Q, \\
\left.\mathbf{p}\right|_{(0, T) \times \partial \Omega}=0, \quad \frac{\partial \mathbf{p}}{\partial x_{0}}(0, \cdot)=\frac{\partial \mathbf{p}}{\partial x_{0}}(T, \cdot)=0, \\
\mathbf{p}=\frac{1}{s^{2}} \mathbf{v}_{2} \mathrm{e}^{-2 s \phi} \quad \text { in } Q_{\omega}, \quad \frac{\partial \mathbf{p}}{\partial x_{0}}=-\mathbf{v}_{1} \mathrm{e}^{-2 s \phi} \quad \text { in } Q_{\omega}, \\
P \mathbf{z}=\mathbf{u} \mathbf{e}^{2 s \phi}+\frac{\partial \mathbf{v}_{1}}{\partial x_{0}}+\mathbf{v}_{2} \quad \text { in } Q, \operatorname{supp} \mathbf{v}_{j} \subset \overline{Q_{\omega}}, \quad j \in\{1,2\}, \\
\left.\mathbf{z}\right|_{(0, T) \times \partial \Omega}=0, \quad \frac{\partial \mathbf{z}}{\partial x_{0}}(0, \cdot)=\frac{\partial \mathbf{z}}{\partial x_{0}}(T, \cdot)=0,
\end{gathered}
$$


and the following estimate holds true:

$$
\left\|\mathbf{z} \mathrm{e}^{-s \phi}\right\|_{\mathcal{X}}^{2}+\left\|\frac{\partial \mathbf{v}_{1}}{\partial x_{0}} \mathrm{e}^{-s \phi}\right\|_{\left(L^{2}\left(Q_{\omega}\right)\right)^{2}}^{2}+s^{2}\left\|\mathbf{v}_{1} \mathrm{e}^{-s \phi}\right\|_{\left(L^{2}\left(Q_{\omega}\right)\right)^{2}}^{2}+\left\|\mathbf{v}_{2} \mathrm{e}^{-s \phi}\right\|_{\left(L^{2}\left(Q_{\omega}\right)\right)^{2}}^{2} \leq C_{1}\left\|\mathbf{u} \mathrm{e}^{s \phi}\right\|_{\left(L^{2}(Q)\right)^{2}}^{2}
$$

Proof of Lemma 9.1. The proof is done along the standard argument (e.g., [38]) and for completeness we will give it. For any $\varepsilon \in(0,1)$, we consider the following extremal problem

$$
\begin{gathered}
J_{\varepsilon}\left(\mathbf{z}, \mathbf{v}_{1}, \mathbf{v}_{2}, \mathbf{w}\right)=\frac{1}{2} \int_{Q}|\mathbf{z}|^{2} \mathrm{e}^{-2 s \phi} \mathrm{d} x+\frac{1}{2} \int_{Q} m_{\varepsilon}\left(\left|\mathbf{v}_{1}\right|^{2}+\frac{\left|\mathbf{v}_{2}\right|^{2}}{s^{2}}\right) \mathrm{e}^{-2 s \phi} \mathrm{d} x+\frac{1}{2 \varepsilon} \int_{Q}|\mathbf{w}|^{2} \mathrm{~d} x \longrightarrow \inf , \\
P \mathbf{z}=\frac{\partial \mathbf{v}_{1}}{\partial x_{0}}+\mathbf{v}_{2}+\mathbf{u} \mathrm{e}^{2 s \phi}+\mathbf{w} \quad \text { in } Q \\
\left.\mathbf{z}\right|_{(0, T) \times \partial \Omega}=0, \quad \frac{\partial \mathbf{z}}{\partial x_{0}}\left(0, x^{\prime}\right)=\frac{\partial \mathbf{z}}{\partial x_{0}}\left(T, x^{\prime}\right)=0,
\end{gathered}
$$

where $m_{\varepsilon} \in C^{2}(\bar{\Omega}), m_{\varepsilon}\left(x^{\prime}\right)>0$ on $\bar{\Omega}$,

$$
m_{\varepsilon}\left(x^{\prime}\right)= \begin{cases}1, & \text { for } x \in \omega \\ \frac{1}{\varepsilon}, & \text { for } \operatorname{dist}(x, \omega) \geq \frac{1}{\ln \frac{1}{\varepsilon}}\end{cases}
$$

Denote by $\left(\widehat{\mathbf{z}}_{\varepsilon}, \widehat{\mathbf{v}}_{1, \varepsilon}, \widehat{\mathbf{v}}_{2, \varepsilon}, \widehat{\mathbf{w}}_{\varepsilon}\right)$ a solution to extremal problem $(9.10)-(9.12)$.

Remark. We understand equation (9.11) and the boundary Conditions (9.12) in the sense of the equality:

$$
(\mathbf{z}, P \delta)_{\left(L^{2}(Q)\right)^{2}}=-\left(\mathbf{v}_{1}, \partial_{x_{0}} \delta\right)_{\left(L^{2}(Q)\right)^{2}}+\left(\mathbf{v}_{2}+\mathbf{u}^{2 s \phi}+\mathbf{w}, \delta\right)_{\left(L^{2}(Q)\right)^{2}}
$$

for any $\delta \in\left(H^{1}(Q)\right)^{2}$ satisfying $P \delta \in\left(L^{2}(Q)\right)^{2},\left.\delta\right|_{(0, T) \times \partial \Omega}=0, \frac{\partial \delta}{\partial x_{0}}(0,)=.\frac{\partial \delta}{\partial x_{0}}(T,)=$.0 . If $\mathbf{z}, \mathbf{v}_{1}$ are regular, then $\frac{\partial \mathbf{z}}{\partial x_{0}}(0,)-.\mathbf{v}_{1}(0,)=$.0 and $\frac{\partial \mathbf{z}}{\partial x_{0}}(T,)-.\mathbf{v}_{1}(T,)=$.0 .

We have

Proposition 9.1. Under conditions of Theorem 2.2 for all $\mathbf{u} \in\left(L^{2}(Q)\right)^{2}$, there exists a unique solution $\left(\widehat{\mathbf{z}}_{\varepsilon}, \widehat{\mathbf{v}}_{1, \varepsilon}, \widehat{\mathbf{v}}_{2, \varepsilon}, \widehat{\mathbf{w}}_{\varepsilon}\right) \in\left(H^{1}(Q)\right)^{2} \times\left(H^{1}\left(0, T ; L^{2}(\Omega)\right)\right)^{2} \times\left(H^{2}(Q)\right)^{2} \times\left(H^{2}(Q)\right)^{2}$ to problem (9.10)-(9.12). Moreover this solution satisfies the optimality system:

$$
\begin{gathered}
\mathbf{p}_{\varepsilon}(x)=\frac{\widehat{\mathbf{w}}_{\varepsilon}(x)}{\varepsilon} \quad \text { in } Q \\
P \mathbf{p}_{\varepsilon}+\mathrm{e}^{-2 s \phi} \widehat{\mathbf{z}}_{\varepsilon}=0 \quad \text { in } Q \\
\left.\mathbf{p}_{\varepsilon}\right|_{(0, T) \times \partial \Omega}=\left.\widehat{\mathbf{z}}_{\varepsilon}\right|_{(0, T) \times \partial \Omega}=0 \\
\frac{\partial \mathbf{p}_{\varepsilon}}{\partial x_{0}}(0, \cdot)=\frac{\partial \mathbf{p}_{\varepsilon}}{\partial x_{0}}(T, \cdot)=\frac{\partial \widehat{\mathbf{z}}_{\varepsilon}}{\partial x_{0}}(0, \cdot)=\frac{\partial \widehat{\mathbf{z}}_{\varepsilon}}{\partial x_{0}}(T, \cdot)=0, \\
P \widehat{\mathbf{z}}_{\varepsilon}=\frac{\partial \widehat{\mathbf{v}}_{1, \varepsilon}}{\partial x_{0}}+\widehat{\mathbf{v}}_{2, \varepsilon}+\mathbf{u} \mathrm{e}^{2 s \phi}+\widehat{\mathbf{w}}_{\varepsilon} \quad \text { in } Q \\
\frac{\partial \mathbf{p}_{\varepsilon}}{\partial x_{0}}+m_{\varepsilon} \widehat{\mathbf{v}}_{1, \varepsilon} \mathrm{e}^{-2 s \phi}=0 \quad \text { in } Q \\
\mathbf{p}_{\varepsilon}-m_{\varepsilon} \frac{\widehat{\mathbf{v}}_{2, \varepsilon}}{s^{2}} \mathrm{e}^{-2 s \phi}=0 \quad \text { in } Q
\end{gathered}
$$


and the following estimate holds:

$$
\left\|\widehat{\mathbf{z}}_{\varepsilon} \mathrm{e}^{-s \phi}\right\|_{\mathcal{X}}^{2}+\left\|\frac{\partial \widehat{\mathbf{v}}_{1, \varepsilon}}{\partial x_{0}} \mathrm{e}^{-s \phi}\right\|_{\left(L^{2}\left(Q_{\omega}\right)\right)^{2}}^{2}+s^{2}\left\|\widehat{\mathbf{v}}_{1, \varepsilon} \mathrm{e}^{-s \phi}\right\|_{\left(L^{2}\left(Q_{\omega}\right)\right)^{2}}^{2}+\left\|\widehat{\mathbf{v}}_{2, \varepsilon} \mathrm{e}^{-s \phi}\right\|_{\left(L^{2}\left(Q_{\omega}\right)\right)^{2}}^{2} \leq C_{2}\left\|\mathbf{u} \mathrm{e}^{s \phi}\right\|_{\left(L^{2}(Q)\right)^{2}}^{2}
$$

Proof of Proposition 9.1. Since the functional $J_{\varepsilon}$ is strictly convex and the set of admissible elements is a linear space, problem (9.10)-(9.12) has at most one solution. First let us prove that there exists a solution to $(9.10)-(9.12)$ : an element $\left(\widehat{\mathbf{z}}, \widehat{\mathbf{v}}_{1}, \widehat{\mathbf{v}}_{2}, \widehat{\mathbf{w}}\right)$ in the space $\left(L^{2}(Q)\right)^{8}$. Obviously $\left(0,0,0,-\mathbf{u e}{ }^{-2 s \phi}\right)$ is an admissible element and so the set of an admissible elements is not empty. Hence there exists a minimizing sequence $\left\{\left(\widehat{\mathbf{z}}_{j, \varepsilon}, \widehat{\mathbf{v}}_{1, j, \varepsilon}, \widehat{\mathbf{v}}_{2, j, \varepsilon}, \widehat{\mathbf{w}}_{j, \varepsilon}\right)\right\}_{j=1}^{\infty}$ such that

$$
\left(\widehat{\mathbf{z}}_{j, \varepsilon}, \widehat{\mathbf{v}}_{1, j, \varepsilon}, \widehat{\mathbf{v}}_{2, j, \varepsilon}, \widehat{\mathbf{w}}_{j, \varepsilon}\right) \rightarrow\left(\widehat{\mathbf{z}}_{\varepsilon}, \widehat{\mathbf{v}}_{1, \varepsilon}, \widehat{\mathbf{v}}_{2, \varepsilon}, \widehat{\mathbf{w}}_{\varepsilon}\right) \quad \text { weakly in }\left(L^{2}(Q)\right)^{8} .
$$

Passing to the limit in (9.11), (9.12) and using (9.20), we obtain that $\left(\widehat{\mathbf{z}}_{\varepsilon}, \widehat{\mathbf{v}}_{1, \varepsilon}, \widehat{\mathbf{v}}_{2, \varepsilon}, \widehat{\mathbf{w}}_{\varepsilon}\right)$ is an admissible element. On the other hand, since the functional $J_{\varepsilon}$ is lower semi-continuous with respect to the weak convergence in $\left(L^{2}(Q)\right)^{8}$, this element is a solution to problem $(9.10)-(9.12)$.

In order to obtain optimality system (9.13)-(9.18), we introduce the function $\mathbf{q}\left(\delta_{1}, \delta_{2}, \delta_{3}\right)=J_{\varepsilon}\left(\widehat{\mathbf{z}}_{\varepsilon}+\delta_{1} d_{1}, \widehat{\mathbf{v}}_{1, \varepsilon}+\right.$ $\left.\delta_{2} d_{2}, \widehat{\mathbf{v}}_{2, \varepsilon}+\delta_{3} d_{3}, r\left(\delta_{1}, \delta_{2}, \delta_{3}\right)\right)$, where $d_{1} \in\left(L^{2}(Q)\right)^{2}$ with $P d_{1} \in\left(L^{2}(Q)\right)^{2}, d_{2} \in\left(H^{1}(Q)\right)^{2}, d_{3} \in\left(L^{2}(Q)\right)^{2}$,

$$
r\left(\delta_{1}, \delta_{2}, \delta_{3}\right)=P\left(\widehat{\mathbf{z}}_{\varepsilon}+\delta_{1} d_{1}\right)-\left(\frac{\partial}{\partial x_{0}}\left(\widehat{\mathbf{v}}_{1, \varepsilon}+\delta_{2} d_{2}\right)+\widehat{\mathbf{v}}_{2, \varepsilon}+\delta_{3} d_{3}\right)-\mathbf{u} \mathrm{e}^{2 s \phi} .
$$

Obviously the function $\mathbf{q}$ attains the minimum in $\mathbb{R}^{3}$ at $(0,0,0)$ if the variation is admissible. Thus $\nabla \mathbf{q}(0,0,0)=$ 0 . Moreover the equalities $\frac{\partial \mathbf{q}}{\partial \delta_{2}}(0,0,0)=\frac{\partial \mathbf{q}}{\partial \delta_{3}}(0,0,0)=0$ imply

$$
\begin{gathered}
-\frac{1}{\varepsilon} \int_{Q} \widehat{\mathbf{w}}_{\varepsilon} \frac{\partial d_{2}}{\partial x_{0}} \mathrm{~d} x+\int_{Q} m_{\varepsilon} \widehat{\mathbf{v}}_{1, \varepsilon} d_{2} \mathrm{e}^{-2 s \phi} \mathrm{d} x=0, \forall d_{2} \in\left(H^{1}(Q)\right)^{2} \text { such that } d_{2}(0, \cdot)=d_{2}(T, \cdot)=0, \\
-\frac{1}{\varepsilon} \int_{Q} \widehat{\mathbf{w}}_{\varepsilon} d_{3} \mathrm{~d} x+\int_{Q} m_{\varepsilon} \frac{\widehat{\mathbf{v}}_{2, \varepsilon} d_{3}}{s^{2}} \mathrm{e}^{-2 s \phi} \mathrm{d} x=0, \quad \forall d_{3} \in\left(L^{2}(Q)\right)^{2} .
\end{gathered}
$$

On the other hand, these equalities are equivalent to

$$
\begin{gathered}
\frac{1}{\varepsilon} \frac{\partial \widehat{\mathbf{w}}_{\varepsilon}}{\partial x_{0}}+m_{\varepsilon} \widehat{\mathbf{v}}_{1, \varepsilon} \mathrm{e}^{-2 s \phi}=0 \quad \text { in } Q, \\
\frac{\widehat{\mathbf{w}}_{\varepsilon}}{\varepsilon}-m_{\varepsilon} \frac{\widehat{\mathbf{v}}_{2, \varepsilon}}{s^{2}} \mathrm{e}^{-2 s \phi}=0 \quad \text { in } Q .
\end{gathered}
$$

By the equality $\frac{\partial \mathbf{q}}{\partial \delta_{1}}(0,0,0)=0$, we obtain

$$
\left(\frac{\widehat{\mathbf{w}}_{\varepsilon}}{\varepsilon}, P d_{1}\right)_{\left(L^{2}(Q)\right)^{2}}+\int_{Q} \widehat{\mathbf{z}}_{\varepsilon} d_{1} \mathrm{e}^{-2 s \phi} \mathrm{d} x=0, \quad \forall d_{1} \in X
$$

where $X=\left\{d_{1} \in L^{2}\left(0, T ;\left(H^{2}(\Omega)\right)^{2}\right) ; P d_{1} \in\left(L^{2}(Q)\right)^{2},\left.d_{1}\right|_{(0, T) \times \partial \Omega}=0, \frac{\partial d_{1}}{\partial x_{0}}(0, \cdot)=\frac{\partial d_{1}}{\partial x_{0}}(T, \cdot)=0\right\}$.

Since $\widehat{\mathbf{v}}_{1, \varepsilon} \in\left(L^{2}(Q)\right)^{2}$, we obtain immediately from $(9.21)$ that $\frac{\partial \widehat{\mathbf{w}}_{\varepsilon}}{\partial x_{0}} \in\left(L^{2}(Q)\right)^{2}$. Since $d_{1}(0, \cdot)$ and $d_{1}(T, \cdot)$ can be chosen arbitrarily, it follows from (9.23) that

$$
\frac{\partial \widehat{\mathbf{w}}_{\varepsilon}}{\partial x_{0}}(0, \cdot)=\frac{\partial \widehat{\mathbf{w}}_{\varepsilon}}{\partial x_{0}}(T, \cdot)=0,\left.\quad \widehat{\mathbf{w}}_{\varepsilon}\right|_{(0, T) \times \partial \Omega}=0 .
$$


Introducing the function $\mathbf{p}_{\varepsilon}$ by formula (9.13), in terms of (9.21)-(9.23), we immediately obtain equalities (9.17), (9.18) and (9.14), (9.15). Equation (9.17) implies $\frac{\partial \mathbf{p}_{\varepsilon}}{\partial x_{0}} \in\left(L^{2}(Q)\right)^{2}$. From (9.14), (9.15) we obtain $\mathbf{p}_{\varepsilon} \in\left(H^{1}(Q)\right)^{2}$.

Next we will show that $\mathbf{p}_{\varepsilon} \in\left(H^{2}(Q)\right)^{2}$. We extend $\mathbf{p}_{\varepsilon}$ on the set $[-T, 2 T] \times \Omega$ by the formula: $\mathbf{p}_{\varepsilon}\left(x_{0}, x^{\prime}\right)=$ $\mathbf{p}_{\varepsilon}\left(-x_{0}, x^{\prime}\right)$ for $x \in[-T, 0] \times \Omega$ and $\mathbf{p}_{\varepsilon}\left(x_{0}, x^{\prime}\right)=\mathbf{p}_{\varepsilon}\left(2 T-x_{0}, x^{\prime}\right)$ for $\left(x_{0}, x^{\prime}\right) \in[T, 2 T] \times \Omega$. In the same way, we extend $-\widehat{\mathbf{z}}_{\varepsilon} \mathrm{e}^{-2 s \phi}$ on the domain $[-T, 2 T] \times \Omega$ and denote the extended function by $\widetilde{\mathbf{f}}$. Since $\frac{\partial \phi}{\partial x_{0}}\left(T, x^{\prime}\right)<0$ for all $x^{\prime} \in \bar{\Omega}$ and $\frac{\partial \phi}{\partial x_{0}}\left(0, x^{\prime}\right)>0$ for all $x^{\prime} \in \bar{\Omega}$ by (2.10), there exists $\delta>0$ such that we can continue the function $\phi$ on $[-\delta, T+\delta] \times \Omega$ up to a $C^{3}$-function such that $\frac{\partial \phi}{\partial x_{0}}(x)<0$ for all $x \in[T, T+\delta] \times \bar{\Omega}$ and $\frac{\partial \phi}{\partial x_{0}}(x)>0$ for all $x \in[-\delta, 0] \times \bar{\Omega}$. By (9.14), we have

$$
P \mathbf{p}_{\varepsilon}=\widetilde{\mathbf{f}} \quad \text { in } \widetilde{Q} \equiv[-\delta, T+\delta] \times \Omega .
$$

Also Condition 2.1 for the function $\phi$ holds true if we replace the domains $Q, Q_{\omega}$ by $\widetilde{Q},[-\delta, T+\delta] \times \omega$ respectively.

Let $D_{h} f=\frac{f\left(x_{0}+h, x^{\prime}\right)-f(x)}{h}$ and $D_{\bar{h}} f=\frac{f(x)-f\left(x_{0}-h, x^{\prime}\right)}{h}$. For the function $D_{h} D_{\bar{h}} \mathbf{p}_{\varepsilon}$, we have

$$
\left.\frac{\partial}{\partial x_{0}} D_{h} D_{\bar{h}} \mathbf{p}_{\varepsilon}\right|_{x_{0}=0}=\left.\frac{\partial}{\partial x_{0}} D_{h} D_{\bar{h}} \mathbf{p}_{\varepsilon}\right|_{x_{0}=T}=0 .
$$

Note that $P D_{h} D_{\bar{h}} \mathbf{p}_{\varepsilon}=D_{h} D_{\bar{h}} \widetilde{\mathbf{f}}$. Hence

$$
\left(\widehat{\mathbf{z}}_{\varepsilon}, D_{h} D_{\bar{h}} \widetilde{\mathbf{f}}\right)_{\left(L^{2}(Q)\right)^{2}}=-\left(\widehat{\mathbf{v}}_{1, \varepsilon}, \partial_{x_{0}} D_{h} D_{\bar{h}} \mathbf{p}_{\varepsilon}\right)_{\left(L^{2}(Q)\right)^{2}}+\left(\widehat{\mathbf{v}}_{2, \varepsilon}+\mathbf{u} e^{2 s \phi}+\widehat{\mathbf{w}}_{\varepsilon}, D_{h} D_{\bar{h}} \mathbf{p}_{\varepsilon}\right)_{\left(L^{2}(Q)\right)^{2}} .
$$

Using (9.17), (9.18) and the definition of the function $\widetilde{\mathbf{f}}$, we have

$$
\begin{aligned}
& \frac{1}{2}\left(D_{h} \widehat{\mathbf{z}}_{\varepsilon}, D_{h}\left(e^{-2 s \phi} \widehat{\mathbf{z}}_{\varepsilon}\right)\right)_{\left(L^{2}(Q)\right)^{2}}+\frac{1}{2}\left(D_{\bar{h}} \widehat{\mathbf{z}}_{\varepsilon}, D_{\bar{h}}\left(e^{-2 s \phi} \widehat{\mathbf{z}}_{\varepsilon}\right)\right)_{\left(L^{2}(Q)\right)^{2}} \\
+ & \frac{1}{2}\left(D_{h} \widehat{\mathbf{v}}_{1, \varepsilon}, D_{h}\left(m_{\varepsilon} e^{-2 s \phi} \widehat{\mathbf{v}}_{1, \varepsilon}\right)\right)_{\left(L^{2}(Q)\right)^{2}}+\frac{1}{2}\left(D_{\bar{h}} \widehat{\mathbf{v}}_{1, \varepsilon}, D_{\bar{h}}\left(m_{\varepsilon} e^{-2 s \phi} \widehat{\mathbf{v}}_{1, \varepsilon}\right)\right)_{\left(L^{2}(Q)\right)^{2}} \\
+ & \frac{1}{2}\left(D_{h} \widehat{\mathbf{v}}_{2, \varepsilon}, D_{h}\left(s^{-2} m_{\varepsilon} e^{-2 s \phi} \widehat{\mathbf{v}}_{2, \varepsilon}\right)\right)_{\left(L^{2}(Q)\right)^{2}}+\frac{1}{2}\left(D_{\bar{h}} \widehat{\mathbf{v}}_{2, \varepsilon}, D_{\bar{h}}\left(s^{-2} m_{\varepsilon} e^{-2 s \phi} \widehat{\mathbf{v}}_{2, \varepsilon}\right)\right)_{\left(L^{2}(Q)\right)^{2}} \\
+ & \frac{1}{2 \varepsilon}\left(D_{h} \mathbf{w}_{\varepsilon}, D_{h} \mathbf{w}_{\varepsilon}\right)_{\left(L^{2}(Q)\right)^{2}}+\frac{1}{2 \varepsilon}\left(D_{\bar{h}} \mathbf{w}_{\varepsilon}, D_{\bar{h}} \mathbf{w}_{\varepsilon}\right)_{\left(L^{2}(Q)\right)^{2}} \\
= & \left(\mathbf{u} e^{2 s \phi}, D_{h} D_{\bar{h}} \mathbf{p}_{\varepsilon}\right)_{\left(L^{2}(Q)\right)^{2}}
\end{aligned}
$$

Hence

$$
\begin{aligned}
& \left\|D_{h} \widehat{\mathbf{z}}_{\varepsilon}\right\|_{\left(L^{2}(Q)\right)^{2}}+\left\|D_{h} \widehat{\mathbf{v}}_{1, \varepsilon}\right\|_{\left(L^{2}(Q)\right)^{2}}+\left\|D_{h} \widehat{\mathbf{v}}_{2, \varepsilon}\right\|_{\left(L^{2}(Q)\right)^{2}} \\
\leq & C_{2}^{\prime}\left(\left\|D_{h} \mathbf{u}\right\|_{\left(L^{2}(Q)\right)^{2}}+\left\|\left(\widehat{\mathbf{z}}_{\varepsilon}, \widehat{\mathbf{v}}_{1, \varepsilon}, \widehat{\mathbf{v}}_{2, \varepsilon}\right)\right\|_{\left.\left(L^{2}(Q)\right)^{6}\right)}\right.
\end{aligned}
$$

where the constant $C_{2}^{\prime}>0$ is independent of $h$. Therefore

$$
\left(\partial_{x_{0}} \widehat{\mathbf{z}}_{\varepsilon}, \partial_{x_{0}} \widehat{\mathbf{v}}_{1, \varepsilon}, \partial_{x_{0}} \widehat{\mathbf{v}}_{2, \varepsilon}\right) \in\left(L^{2}(Q)\right)^{6} .
$$

Equations (9.11) - (9.18) imply that $\widehat{\mathbf{z}}_{\varepsilon} \in\left(H^{1}(Q)\right)^{2}$ and $\mathbf{p}_{\varepsilon} \in\left(H^{2}(Q)\right)^{2}$.

Let $\chi_{1} \in C_{0}^{\infty}(-\delta, T+\delta)$ be a cut-off function such that $\left.\chi_{1}\right|_{\left[-\frac{\delta}{2}, T+\frac{\delta}{2}\right]}=1$. Then

$$
P\left(\chi_{1} \mathbf{p}_{\varepsilon}\right)=\chi_{1} \widetilde{\mathbf{f}}-\left[\chi_{1}, P\right] \mathbf{p}_{\varepsilon} \text { in } \widetilde{Q},
$$

where $\operatorname{supp}\left[\chi_{1}, P\right] \mathbf{p}_{\varepsilon} \subset\left(\left[T+\frac{\delta}{2}, T+\delta\right] \times \bar{\Omega}\right) \cup\left(\left[-\delta,-\frac{\delta}{2}\right] \times \bar{\Omega}\right)$. We will apply Carleman estimate (2.9) to equation (9.25). 
For this, we observe that

$$
\begin{aligned}
& \left\|\widetilde{\mathbf{f}} e^{s \phi}\right\|_{L^{2}\left(-\delta, T+\delta ;\left(L^{2}(\Omega)\right)^{2}\right)} \leq C_{3}\left\|\mathbf{z}_{\varepsilon} \mathrm{e}^{-s \phi}\right\|_{\left(L^{2}(Q)\right)^{2}}, \\
& \left\|\left(\left[\chi_{1}, P\right] \mathbf{p}_{\varepsilon}\right) \mathrm{e}^{s \phi}\right\|_{L^{2}\left(-\delta, T+\delta ;\left(L^{2}(\Omega)\right)^{2}\right)} \leq \frac{C_{4}}{s}\left\|\mathbf{p}_{\varepsilon} \mathrm{e}^{s \phi}\right\|_{\mathcal{X}} .
\end{aligned}
$$

Moreover, by a way similar to Appendix II in [26] (i.e., the final step of the proof of Lem. 2.3 in [26]), we can prove that at the right hand side of (2.9), we can replace the integral over $Q_{\omega}$ by the following integral

$$
\int_{Q_{\omega}}\left(\left|\frac{\partial^{2} \mathbf{u}}{\partial x_{0}^{2}}\right|^{2}+s^{2}\left|\frac{\partial \mathbf{u}}{\partial x_{0}}\right|^{2}+s^{4}|\mathbf{u}|^{2}\right) \mathrm{e}^{2 s \phi} \mathrm{d} x .
$$

Note that thanks to the choice of the extension of the function $\phi$, we have

$$
\begin{aligned}
\int_{(-\delta, T+\delta) \times \omega}\left(\left|\frac{\partial^{2}\left(\chi_{1} \mathbf{p}_{\varepsilon}\right)}{\partial x_{0}^{2}}\right|^{2}+s^{2}\left|\frac{\partial\left(\chi_{1} \mathbf{p}_{\varepsilon}\right)}{\partial x_{0}}\right|^{2}+s^{4}\left|\chi_{1} \mathbf{p}_{\varepsilon}\right|^{2}\right) \mathrm{e}^{2 s \phi} \mathrm{d} x & \\
& \leq C_{5} \int_{Q_{\omega}}\left(\left|\frac{\partial^{2} \mathbf{p}_{\varepsilon}}{\partial x_{0}^{2}}\right|^{2}+s^{2}\left|\frac{\partial \mathbf{p}_{\varepsilon}}{\partial x_{0}}\right|^{2}+s^{4}\left|\mathbf{p}_{\varepsilon}\right|^{2}\right) \mathrm{e}^{2 s \phi} \mathrm{d} x .
\end{aligned}
$$

In fact, let us denote the left and the right hand sides of (9.27) respectively by $I_{1}$ and $I_{2}$. First we can easily see

$$
I_{1} \leq C_{5}^{\prime} \int_{(-\delta, T+\delta) \times \omega}\left(\left|\frac{\partial^{2} \mathbf{p}_{\varepsilon}}{\partial x_{0}^{2}}\right|^{2}+s^{2}\left|\frac{\partial \mathbf{p}_{\varepsilon}}{\partial x_{0}}\right|^{2}+s^{4}\left|\mathbf{p}_{\varepsilon}\right|^{2}\right) \mathrm{e}^{2 s \phi} \mathrm{d} x .
$$

On the other hand, since $\mathbf{p}_{\varepsilon}\left(x_{0}, x^{\prime}\right)=\mathbf{p}_{\varepsilon}\left(-x_{0}, x^{\prime}\right),-\delta \leq x^{\prime} \leq 0$ by the extension, we have

$$
\begin{aligned}
\int_{-\delta}^{0} \int_{\omega}\left(\left|\frac{\partial^{2} \mathbf{p}_{\varepsilon}}{\partial x_{0}^{2}}\right|^{2}+s^{2}\left|\frac{\partial \mathbf{p}_{\varepsilon}}{\partial x_{0}}\right|^{2}+s^{4}\left|\mathbf{p}_{\varepsilon}\right|^{2}\right) & \mathrm{e}^{2 s \phi\left(x_{0}, x^{\prime}\right)} \mathrm{d} x_{0} \mathrm{~d} x^{\prime}= \\
& \int_{0}^{\delta} \int_{\omega}\left(\left|\frac{\partial^{2} \mathbf{p}_{\varepsilon}}{\partial x_{0}^{2}}\right|^{2}+s^{2}\left|\frac{\partial \mathbf{p}_{\varepsilon}}{\partial x_{0}}\right|^{2}+s^{4}\left|\mathbf{p}_{\varepsilon}\right|^{2}\right) \mathrm{e}^{2 s \phi\left(-x_{0}, x^{\prime}\right)} \mathrm{d} x_{0} \mathrm{~d} x^{\prime} .
\end{aligned}
$$

By (2.10), we have $\partial_{x_{0}} \phi\left(0, x^{\prime}\right)>0$. Therefore, for all sufficiently small $\delta>0$, we obtain $\partial_{x_{0}} \phi(x)>0$ for all $x_{0} \in[-\delta, \delta]$. This implies $e^{2 s \phi\left(-x_{0}, x^{\prime}\right)} \leq e^{2 s \phi\left(x_{0}, x^{\prime}\right)}$ for $0<x_{0}<\delta$. Hence

$$
\begin{aligned}
\int_{0}^{\delta} \int_{\omega}\left(\left|\frac{\partial^{2} \mathbf{p}_{\varepsilon}}{\partial x_{0}^{2}}\right|^{2}+s^{2}\left|\frac{\partial \mathbf{p}_{\varepsilon}}{\partial x_{0}}\right|^{2}+s^{4}\left|\mathbf{p}_{\varepsilon}\right|^{2}\right) \mathrm{e}^{2 s \phi\left(-x_{0}, x^{\prime}\right)} \mathrm{d} x_{0} \mathrm{~d} x^{\prime} \\
\quad \leq \int_{0}^{\delta} \int_{\omega}\left(\left|\frac{\partial^{2} \mathbf{p}_{\varepsilon}}{\partial x_{0}^{2}}\right|^{2}+s^{2}\left|\frac{\partial \mathbf{p}_{\varepsilon}}{\partial x_{0}}\right|^{2}+s^{4}\left|\mathbf{p}_{\varepsilon}\right|^{2}\right) \mathrm{e}^{2 s \phi\left(x_{0}, x^{\prime}\right)} \mathrm{d} x_{0} \mathrm{~d} x^{\prime} \leq I_{2} .
\end{aligned}
$$

We can similarly estimate

$$
\int_{T}^{T+\delta} \int_{\omega}\left(\left|\frac{\partial^{2} \mathbf{p}_{\varepsilon}}{\partial x_{0}^{2}}\right|^{2}+s^{2}\left|\frac{\partial \mathbf{p}_{\varepsilon}}{\partial x_{0}}\right|^{2}+s^{4}\left|\mathbf{p}_{\varepsilon}\right|^{2}\right) \mathrm{e}^{2 s \phi} \mathrm{d} x_{0} \mathrm{~d} x^{\prime}
$$

Thus the verification of (9.27) is complete. 
Using equations (9.17), (9.18), (9.24) and estimate (9.27), by Theorem 2.1, we obtain

$$
\sum_{|\alpha|=2}\left\|\left(\partial_{x}^{\alpha} \mathbf{p}_{\varepsilon}\right) \mathrm{e}^{s \phi}\right\|_{\left(L^{2}(Q)\right)^{2}}^{2}+s^{2}\left\|\mathbf{p}_{\varepsilon} \mathrm{e}^{s \phi}\right\|_{\mathcal{X}}^{2} \leq C_{6} M\left(\widehat{\mathbf{z}}_{\varepsilon}, \widehat{\mathbf{v}}_{1, \varepsilon}, \widehat{\mathbf{v}}_{2, \varepsilon}\right),
$$

where we set

$$
M\left(\widehat{\mathbf{z}}_{\varepsilon}, \widehat{\mathbf{v}}_{1, \varepsilon}, \widehat{\mathbf{v}}_{2, \varepsilon}\right)=\left\|\widehat{\mathbf{z}}_{\varepsilon} \mathrm{e}^{-s \phi}\right\|_{\mathcal{X}}^{2}+\int_{Q_{\omega}}\left(\left|\frac{\partial \widehat{\mathbf{v}}_{1, \varepsilon}}{\partial x_{0}}\right|^{2}+s^{2}\left|\widehat{\mathbf{v}}_{1, \varepsilon}\right|^{2}+\left|\widehat{\mathbf{v}}_{2, \varepsilon}\right|^{2}\right) \mathrm{e}^{-2 s \phi} \mathrm{d} x
$$

By (9.14)-(9.18) and integration by parts, we have

$$
\left(\frac{\partial \widehat{\mathbf{v}}_{1, \varepsilon}}{\partial x_{0}}+\widehat{\mathbf{v}}_{2, \varepsilon}+\mathbf{u} e^{2 s \phi}+\widehat{\mathbf{w}}_{\varepsilon}, \mathbf{p}_{\varepsilon}\right)_{\left(L^{2}(Q)\right)^{2}}=\left(P \widehat{\mathbf{z}}_{\varepsilon}, \mathbf{p}_{\varepsilon}\right)_{\left(L^{2}(Q)\right)^{2}}=\left(\widehat{\mathbf{z}}_{\varepsilon}, P \mathbf{p}_{\varepsilon}\right)_{\left(L^{2}(Q)\right)^{2}}=-\left(\widehat{\mathbf{z}}_{\varepsilon}, \widehat{\mathbf{z}}_{\varepsilon} \mathrm{e}^{-2 s \phi}\right)_{\left(L^{2}(Q)\right)^{2}} .
$$

Therefore, taking the scalar product of (9.16) and $\mathbf{p}_{\varepsilon}$ in $\left(L^{2}(Q)\right)^{2}$ and using (9.17) and (9.18), we obtain

$$
2 J_{\varepsilon}\left(\widehat{\mathbf{z}}_{\varepsilon}, \widehat{\mathbf{v}}_{1, \varepsilon}, \widehat{\mathbf{v}}_{2, \varepsilon}, \widehat{\mathbf{w}}_{\varepsilon}\right)=-\frac{1}{2} \int_{Q}\left(\mathbf{u} \mathrm{e}^{2 s \phi}, \mathbf{p}_{\varepsilon}\right) \mathrm{d} x
$$

By (9.28), we obtain from this inequality that

$$
s^{2} J_{\varepsilon}\left(\widehat{\mathbf{z}}_{\varepsilon}, \widehat{\mathbf{v}}_{1, \varepsilon}, \widehat{\mathbf{v}}_{2, \varepsilon}, \widehat{\mathbf{w}}_{\varepsilon}\right) \leq C_{7}\left\|\mathbf{u} e^{s \phi}\right\|_{\left(L^{2}(Q)\right)^{2}} M\left(\widehat{\mathbf{z}}_{\varepsilon}, \widehat{\mathbf{v}}_{1, \varepsilon}, \widehat{\mathbf{v}}_{2, \varepsilon}\right)^{\frac{1}{2}} .
$$

Next we differentiate equations (9.14) and (9.16) with respect to the variable $x_{0}$ :

$$
\begin{gathered}
P \frac{\partial \mathbf{p}_{\varepsilon}}{\partial x_{0}}=\frac{\partial}{\partial x_{0}} \widetilde{\mathbf{f}} \quad \text { in } Q \\
P \frac{\partial \widehat{\mathbf{z}}_{\varepsilon}}{\partial x_{0}}=\frac{\partial^{2} \widehat{\mathbf{v}}_{1, \varepsilon}}{\partial x_{0}^{2}}+\frac{\partial \widehat{\mathbf{v}}_{2, \varepsilon}}{\partial x_{0}}+\frac{\partial\left(\mathbf{u} e^{2 s \phi}\right)}{\partial x_{0}}+\frac{\partial \widehat{\mathbf{w}}_{\varepsilon}}{\partial x_{0}} \quad \text { in } Q .
\end{gathered}
$$

Taking the scalar product of (9.31) and $\frac{\partial \mathbf{p}_{\varepsilon}}{\partial x_{0}}$ in $\left(L^{2}(Q)\right)^{2}$ and integrating by parts, in terms of (9.14)-(9.18), we similarly obtain

$$
\begin{aligned}
2 J_{\varepsilon}\left(\frac{\partial \widehat{\mathbf{z}}_{\varepsilon}}{\partial x_{0}}, \frac{\partial \widehat{\mathbf{v}}_{1, \varepsilon}}{\partial x_{0}}, \frac{\partial \widehat{\mathbf{v}}_{2, \varepsilon}}{\partial x_{0}}, \frac{\partial \widehat{\mathbf{w}}_{\varepsilon}}{\partial x_{0}}\right)=\int_{Q}\left\{\left(\mathbf{u} \mathrm{e}^{2 s \phi}, \frac{\partial^{2} \mathbf{p}_{\varepsilon}}{\partial x_{0}^{2}}\right)+2 s \frac{\partial \phi}{\partial x_{0}}\left(\frac{\partial \widehat{\mathbf{z}}_{\varepsilon}}{\partial x_{0}}, \widehat{\mathbf{z}}_{\varepsilon}\right) \mathrm{e}^{-2 s \phi}\right. \\
\left.\quad+2 s \frac{\partial \phi}{\partial x_{0}} m_{\varepsilon}\left(\frac{\partial \widehat{\mathbf{v}}_{1, \varepsilon}}{\partial x_{0}}, \widehat{\mathbf{v}}_{1, \varepsilon}\right) \mathrm{e}^{-2 s \phi}+\frac{2 m_{\varepsilon}}{s} \frac{\partial \phi}{\partial x_{0}}\left(\frac{\partial \widehat{\mathbf{v}}_{2, \varepsilon}}{\partial x_{0}}, \widehat{\mathbf{v}}_{2, \varepsilon}\right) \mathrm{e}^{-2 s \phi}\right\} \mathrm{d} x .
\end{aligned}
$$

This equality and (9.28), (9.29) imply

$$
J_{\varepsilon}\left(\frac{\partial \widehat{\mathbf{z}}_{\varepsilon}}{\partial x_{0}}, \frac{\partial \widehat{\mathbf{v}}_{1, \varepsilon}}{\partial x_{0}}, \frac{\partial \widehat{\mathbf{v}}_{2, \varepsilon}}{\partial x_{0}}, \frac{\partial \widehat{\mathbf{w}}_{\varepsilon}}{\partial x_{0}}\right) \leq C_{8}\left\|\mathbf{u} \mathrm{e}^{s \phi}\right\|_{\left(L^{2}(Q)\right)^{2}} M\left(\widehat{\mathbf{z}}_{\varepsilon}, \widehat{\mathbf{v}}_{1, \varepsilon}, \widehat{\mathbf{v}}_{2, \varepsilon}\right)^{\frac{1}{2}}
$$


Let $\widetilde{L}$ denote the part of first order of $L_{\lambda, \mu}$, that is, $(\widetilde{L} \mathbf{v})\left(x^{\prime}\right)=\operatorname{div} \mathbf{v}\left(x^{\prime}\right) \nabla_{x^{\prime}} \lambda\left(x^{\prime}\right)+\left(\nabla_{x^{\prime}} \mathbf{v}+\left(\nabla_{x^{\prime}} \mathbf{v}\right)^{T}\right) \nabla_{x^{\prime}} \mu\left(x^{\prime}\right)$. Taking the scalar product of $(9.16)$ with $\widehat{\mathbf{z}}_{\varepsilon} \mathrm{e}^{-2 s \phi}$ in $\left(L^{2}(Q)\right)^{2}$, we obtain

$$
\begin{aligned}
& \int_{Q}\left(\mu\left|\nabla_{x^{\prime}} \widehat{\mathbf{z}}_{\varepsilon}\right|^{2}+(\lambda+\mu)\left(\operatorname{div} \widehat{\mathbf{z}}_{\varepsilon}\right)^{2}\right) \mathrm{e}^{-2 s \phi} \mathrm{d} x-\int_{Q}\left(\widetilde{L} \widehat{\mathbf{z}}_{\varepsilon}, \widehat{\mathbf{z}}_{\varepsilon} \mathrm{e}^{-2 s \phi}\right) \mathrm{d} x \\
& =\int_{Q}\left(\left|\frac{\partial \widehat{\mathbf{z}}_{\varepsilon}}{\partial x_{0}}\right|^{2}-2 s \partial_{x_{0}} \phi\left(\frac{\partial \widehat{\mathbf{z}}_{\varepsilon}}{\partial x_{0}}, \widehat{\mathbf{z}}_{\varepsilon}\right)\right) \mathrm{e}^{-2 s \phi} \mathrm{d} x \\
& \quad+\int_{Q}\left(2 \mu s \sum_{k=1}^{2}\left(\partial_{x_{k}} \widehat{\mathbf{z}}_{\varepsilon},\left(\partial_{x_{k}} \phi\right) \widehat{\mathbf{z}}_{\varepsilon}\right)+2(\lambda+\mu) s\left(\operatorname{div} \widehat{\mathbf{z}}_{\varepsilon}\right)\left(\nabla_{x^{\prime}} \phi, \widehat{\mathbf{z}}_{\varepsilon}\right)\right) \mathrm{e}^{-2 s \phi} \mathrm{d} x \\
& \quad-\int_{Q} \sum_{k=1}^{2}\left(\widehat{\mathbf{z}}_{\varepsilon}, \partial_{x_{k}} \widehat{\mathbf{z}}_{\varepsilon}\right)\left(\partial_{x_{k}} \mu\right) \mathrm{e}^{-2 s \phi} \mathrm{d} x-\int_{Q}\left(\operatorname{div} \widehat{\mathbf{z}}_{\varepsilon}\right)\left(\nabla_{x^{\prime}}(\lambda+\mu), \widehat{\mathbf{z}}_{\varepsilon}\right) \mathrm{e}^{-2 s \phi} \mathrm{d} x \\
& \quad+\int_{Q}\left(\mathbf{u} \mathrm{e}^{2 s \phi}+\widehat{\mathbf{w}}_{\varepsilon}, \widehat{\mathbf{z}}_{\varepsilon}\right) \mathrm{e}^{-2 s \phi} \mathrm{d} x+\int_{Q}\left(\frac{\partial \widehat{\mathbf{v}}_{1, \varepsilon}}{\partial x_{0}}+\widehat{\mathbf{v}}_{2, \varepsilon}, \widehat{\mathbf{z}}_{\varepsilon} \mathrm{e}^{-2 s \phi}\right) \mathrm{d} x .
\end{aligned}
$$

We note that $\left|\partial_{x_{j}} z_{k}\right|\left|z_{\ell}\right| \leq \frac{\delta}{2}\left|\partial_{x_{j}} z_{k}\right|^{2}+\frac{1}{2 \delta}\left|z_{\ell}\right|^{2}$ for any $\delta>0$. Therefore if we take sufficiently small $\delta>0$ and sufficiently large $s>0$, then by (9.28), (9.29) and (9.32), we obtain (9.19). The proof of Proposition 9.1 is complete.

Now we finish the proof of Lemma 9.1. Obviously $\widehat{\mathbf{w}}_{\varepsilon} \rightarrow 0$ in $\left(L^{2}(Q)\right)^{2}$ and $\widehat{\mathbf{v}}_{1, \varepsilon_{k}}, \widehat{\mathbf{v}}_{2, \varepsilon_{k}} \rightarrow 0$ in $\left(L^{2}(Q \backslash\right.$ $\left.\left.Q_{\omega}\right)\right)^{2}$ as $\varepsilon \rightarrow+0$. In terms of (9.19), from the sequence $\left\{\left(\widehat{\mathbf{z}}_{\varepsilon}, \widehat{\mathbf{v}}_{1, \varepsilon}, \widehat{\mathbf{v}}_{2, \varepsilon}, \mathbf{p}_{\varepsilon}\right)\right\}$, one can extract a subsequence $\left\{\left(\widehat{\mathbf{z}}_{\varepsilon_{k}}, \widehat{\mathbf{v}}_{1, \varepsilon_{k}}, \widehat{\mathbf{v}}_{2, \varepsilon_{k}}, \mathbf{p}_{\varepsilon_{k}}\right)\right\}$ such that

$$
\left(\widehat{\mathbf{z}}_{\varepsilon_{k}}, \widehat{\mathbf{v}}_{1, \varepsilon_{k}}, \widehat{\mathbf{v}}_{2, \varepsilon_{k}}, \mathbf{p}_{\varepsilon_{k}}\right) \rightarrow\left(\widehat{\mathbf{z}}, \widehat{\mathbf{v}}_{1}, \widehat{\mathbf{v}}_{2}, \mathbf{p}\right) \text { weakly in } \mathcal{X} \times\left(H^{1}\left(0, T ; L^{2}(\Omega)\right)\right)^{2} \times\left(L^{2}(Q)\right)^{4} .
$$

Thanks to (9.33), we can pass to the limit in (9.14)-(9.18), so that the element $\left(\widehat{\mathbf{z}}, \widehat{\mathbf{v}}_{1}, \widehat{\mathbf{v}}_{2}, \mathbf{p}\right)$ satisfies the equations

$$
\begin{gathered}
P \mathbf{p}+\mathrm{e}^{-2 s \phi} \widehat{\mathbf{z}}=0 \quad \text { in } Q, \\
\left.\mathbf{p}\right|_{(0, T) \times \partial \Omega}=\left.\widehat{\mathbf{z}}\right|_{(0, T) \times \partial \Omega}=0, \\
\frac{\partial \mathbf{p}}{\partial x_{0}}(0, \cdot)=\frac{\partial \mathbf{p}}{\partial x_{0}}(T, \cdot)=\frac{\partial \widehat{\mathbf{z}}}{\partial x_{0}}(0, \cdot)=\frac{\partial \widehat{\mathbf{z}}}{\partial x_{0}}(T, \cdot)=0, \\
P \widehat{\mathbf{z}}=\frac{\partial \widehat{\mathbf{v}}_{1}}{\partial x_{0}}+\widehat{\mathbf{v}}_{2}+\mathbf{u} \mathrm{e}^{2 s \phi} \quad \text { in } Q, \\
\frac{\partial \mathbf{p}}{\partial x_{0}}+\widehat{\mathbf{v}}_{1} \mathrm{e}^{-2 s \phi}=0 \quad \text { in } Q, \\
\mathbf{p}-\frac{\widehat{\mathbf{v}}_{2}}{s^{2}} \mathrm{e}^{-2 s \phi}=0 \quad \text { in } Q, \quad \operatorname{supp} \widehat{\mathbf{v}}_{j} \subset \bar{Q}_{\omega}, \quad j=1,2 .
\end{gathered}
$$

Estimate (9.9) follows from (9.19). Finally we note that $J_{\varepsilon}\left(\widehat{\mathbf{z}}_{\varepsilon}, \widehat{\mathbf{v}}_{1, \varepsilon}, \widehat{\mathbf{v}}_{2, \varepsilon}, \widehat{\mathbf{w}}_{\varepsilon}\right) \leq J\left(\mathbf{z}, \mathbf{v}_{1}, \mathbf{v}_{2}\right)$ for all $\varepsilon \in(0,1)$. Hence $J\left(\widehat{\mathbf{z}}, \widehat{\mathbf{v}}_{1}, \widehat{\mathbf{v}}_{2}\right) \leq J\left(\mathbf{z}, \mathbf{v}_{1}, \mathbf{v}_{2}\right)$, the element $\left(\widehat{\mathbf{z}}, \widehat{\mathbf{v}}_{1}, \widehat{\mathbf{v}}_{2}\right)$ is a solution to extremal problem (9.1)-(9.3). Since a solution to this problem is unique, we have $\left(\widehat{\mathbf{z}}, \widehat{\mathbf{v}}_{1}, \widehat{\mathbf{v}}_{2}\right)=\left(\mathbf{z}, \mathbf{v}_{1}, \mathbf{v}_{2}\right)$. The proof of Lemma 9.1 is complete.

Proof of Theorem 2.2. Taking the scalar product of $(2.1)$ with $\mathbf{z}$ in $\left(L^{2}(Q)\right)^{2}$ and integrating by parts, in terms of $(2.1),(2.2),(9.7)$ and $(9.8)$, we obtain the equality

$$
\left\|\mathbf{u} e^{s \phi}\right\|_{\left(L^{2}(Q)\right)^{2}}^{2}=\int_{Q}(\mathbf{f}, \mathbf{z}) \mathrm{d} x-\int_{Q_{\omega}}\left(\mathbf{u}, \frac{\partial \mathbf{v}_{1}}{\partial x_{0}}+\mathbf{v}_{2}\right) \mathrm{d} x .
$$


Applying (9.9) to this equality and using again an inequality $|a b| \leq \frac{\delta}{2}|a|^{2}+\frac{1}{2 \delta}|b|^{2}$ for any $\delta>0$, we obtain

$$
\int_{Q} s^{2}|\mathbf{u}|^{2} \mathrm{e}^{2 s \phi} \mathrm{d} x \leq C_{9}\left(\left\|\mathbf{f} \mathrm{e}^{s \phi}\right\|_{\left(L^{2}(Q)\right)^{2}}^{2}+\int_{Q_{\omega}}\left(|\nabla \mathbf{u}|^{2}+s^{2}|\mathbf{u}|^{2}\right) \mathrm{e}^{2 s \phi} \mathrm{d} x\right), \quad \forall s \geq s_{0}(\tau) .
$$

In order to estimate the derivatives of first order for the function $\mathbf{u}$, replacing $\mathbf{u}$ by $\frac{\partial \mathbf{u}}{\partial x_{0}}$, we consider extremal problem (9.1)-(9.3). Let $\left(\widetilde{\mathbf{z}}, \widetilde{\mathbf{v}}_{1}, \widetilde{\mathbf{v}}_{2}\right)$ be the corresponding solution. Then Lemma 9.1 yields

$$
\left\|\widetilde{\mathbf{z}} \mathrm{e}^{-s \phi}\right\|_{\mathcal{X}}^{2}+\left\|\frac{\partial \widetilde{\mathbf{v}}_{1}}{\partial x_{0}} \mathrm{e}^{-s \phi}\right\|_{\left(L^{2}\left(Q_{\omega}\right)\right)^{2}}^{2}+\left\|\widetilde{\mathbf{v}}_{2} \mathrm{e}^{-s \phi}\right\|_{\left(L^{2}\left(Q_{\omega}\right)\right)^{2}}^{2} \leq C_{10}\left\|\frac{\partial \mathbf{u}}{\partial x_{0}} \mathrm{e}^{s \phi}\right\|_{\left(L^{2}(Q)\right)^{2}}^{2} .
$$

Since the Lamé coefficients are independent of $x_{0}$, we have

$$
P \frac{\partial \mathbf{u}}{\partial x_{0}}=\frac{\partial \mathbf{f}}{\partial x_{0}} \quad \text { in } Q,\left.\quad \frac{\partial \mathbf{u}}{\partial x_{0}}\right|_{(0, T) \times \partial \Omega}=0, \frac{\partial \mathbf{u}}{\partial x_{0}}(T, \cdot)=\frac{\partial \mathbf{u}}{\partial x_{0}}(0, \cdot)=0 .
$$

Taking the scalar product of $(9.42)$ with $\widetilde{\mathbf{z}}$ in $\left(L^{2}(Q)\right)^{2}$ and integrating by parts, we obtain the equality

$$
\left\|\frac{\partial \mathbf{u}}{\partial x_{0}} \mathrm{e}^{s \phi}\right\|_{\left(L^{2}(Q)\right)^{2}}^{2}=\int_{Q}\left(\frac{\partial \mathbf{f}}{\partial x_{0}}, \widetilde{\mathbf{z}}\right) \mathrm{d} x-\int_{Q_{\omega}}\left(\frac{\partial \mathbf{u}}{\partial x_{0}}, \frac{\partial \widetilde{\mathbf{v}}_{1}}{\partial x_{0}}+\widetilde{\mathbf{v}}_{2}\right) \mathrm{d} x
$$

Applying the inequality $2|a b| \leq \delta|a|^{2}+\frac{1}{\delta}|b|^{2}$ to the second term at the right hand side of this equality, by means of (9.41), we obtain

$$
\int_{Q}\left(\left|\frac{\partial \mathbf{u}}{\partial x_{0}}\right|^{2}+s^{2}|\mathbf{u}|^{2}\right) \mathrm{e}^{2 s \phi} \mathrm{d} x \leq C_{11}\left\{\left\|\mathbf{f e}^{s \phi}\right\|_{\left(L^{2}(Q)\right)^{2}}^{2}+\int_{Q_{\omega}}\left(|\nabla \mathbf{u}|^{2}+s^{2}|\mathbf{u}|^{2}\right) \mathrm{e}^{2 s \phi} \mathrm{d} x\right\}, \quad \forall s \geq s_{0}(\tau)
$$

Finally, taking the scalar product of $(2.1)$ with $\mathbf{u} \mathrm{e}^{2 s \phi}$ in $\left(L^{2}(Q)\right)^{2}$, we obtain

$$
\begin{aligned}
\int_{Q}\left(\mu\left|\nabla_{x^{\prime}} \mathbf{u}\right|^{2}+(\lambda+\mu)(\operatorname{div} \mathbf{u})^{2}\right) \mathrm{e}^{2 s \phi} \mathrm{d} x & =\int_{Q}\left(\left|\frac{\partial \mathbf{u}}{\partial x_{0}}\right|^{2}+2 s \partial_{x_{0}} \phi\left(\frac{\partial \mathbf{u}}{\partial x_{0}}, \mathbf{u}\right)\right) \mathrm{e}^{2 s \phi} \mathrm{d} x \\
-\int_{Q}\left(2 \mu s \sum_{k=1}^{2}\left(\partial_{x_{k}} \mathbf{u},\left(\partial_{x_{k}} \phi\right) \mathbf{u}\right)+\right. & \left.2(\lambda+\mu) s(\operatorname{div} \mathbf{u})\left(\nabla_{x^{\prime}} \phi, \mathbf{u}\right)\right) \mathrm{e}^{2 s \phi} \mathrm{d} x-\int_{Q} \sum_{k=1}^{2}\left(\mathbf{u}, \partial_{x_{k}} \mathbf{u}\right)\left(\partial_{x_{k}} \mu\right) \mathrm{e}^{2 s \phi} \mathrm{d} x \\
& -\int_{Q}(\operatorname{div} \mathbf{u})\left(\nabla_{x^{\prime}}(\lambda+\mu), \mathbf{u}\right) \mathrm{e}^{2 s \phi} \mathrm{d} x+\int_{Q}\left(\widetilde{L} \mathbf{u}, \mathbf{u} \mathrm{e}^{2 s \phi}\right) \mathrm{d} x+\int_{Q}(\mathbf{f}, \mathbf{u}) \mathrm{e}^{2 s \phi} \mathrm{d} x
\end{aligned}
$$

This equality and (9.43) imply (2.11), the conclusion of Theorem 2.2.

Proof of Theorem 2.3. In order to complete the proof, it is sufficient to estimate $\int_{Q}(\mathbf{f}, \mathbf{z}) \mathrm{d} x$ in $(9.39)$ as follows:

$$
\left|\int_{Q}\left(\mathbf{f}_{-1}, \mathbf{z}\right) \mathrm{d} x\right| \leq\left\|\mathbf{f}_{-1} \mathrm{e}^{s \phi}\right\|_{\left(H^{-1}(Q)\right)^{2}}\left\|\mathbf{z} \mathrm{e}^{-s \phi}\right\|_{\left(H^{1}(Q)\right)^{2}} \leq\left\|\mathbf{f}_{-1} \mathrm{e}^{s \phi}\right\|_{\left(H^{-1}(Q)\right)^{2}}\left\|\mathbf{z} \mathrm{e}^{-s \phi}\right\|_{\mathcal{X}}
$$

and

$$
\begin{aligned}
\left|\int_{Q}\left(\partial_{x_{j}} \mathbf{f}_{j}, \mathbf{z}\right) \mathrm{d} x\right|=\left|\int_{Q}\left(\mathbf{f}_{j}, \partial_{x_{j}} \mathbf{z}\right) \mathrm{d} x\right| & \leq\left\|\mathbf{f}_{j} \mathrm{e}^{s \phi}\right\|_{\left(L^{2}(Q)\right)^{2}}\left\|\left(\partial_{x_{j}} \mathbf{z}\right) \mathrm{e}^{-s \phi}\right\|_{\left(L^{2}(Q)\right)^{2}} \\
& \leq C_{12}\left\|\mathbf{f}_{j} \mathrm{e}^{s \phi}\right\|_{\left(L^{2}(Q)\right)^{2}}\left(\left\|\nabla\left(\mathbf{z} \mathrm{e}^{-s \phi}\right)\right\|_{\left(L^{2}(Q)\right)^{2}}+s\left\|\mathbf{z} \mathrm{e}^{-s \phi}\right\|_{\left.\left(L^{2}(Q)\right)^{2}\right)}\right. \\
& \leq C_{13}\left\|\mathbf{f}_{j} \mathrm{e}^{s \phi}\right\|_{\left(L^{2}(Q)\right)^{2}}\left\|\mathbf{z} \mathrm{e}^{-s \phi}\right\|_{\mathcal{X}} .
\end{aligned}
$$


Therefore

$$
\left|\int_{Q}\left(\left(\mathbf{f}_{-1}+\sum_{j=0}^{2} \partial_{x_{j}} \mathbf{f}_{j}\right), \mathbf{z}\right) \mathrm{d} x\right| \leq C_{14}\left(\left\|\mathbf{f}_{-1} \mathrm{e}^{s \phi}\right\|_{\left(H^{-1}(Q)\right)^{2}}+\sum_{j=0}^{2}\left\|\mathbf{f}_{j} \mathrm{e}^{s \phi}\right\|_{\left(L^{2}(Q)\right)^{2}}\right)\left\|\mathbf{z} \mathrm{e}^{-s \phi}\right\|_{\mathcal{X}} .
$$

Then, again by using the inequality $|a b| \leq \frac{\delta}{2}|a|^{2}+\frac{1}{2 \delta}|b|^{2}$ for $\delta>0$, this inequality and estimates (9.9), (9.39) imply (2.12).

\section{Appendix I. Proof of Proposition 5.1}

In order to prove the proposition, it is convenient to use the coordinate $x$ instead of $y$. Moreover it suffices to prove the estimate for an arbitrary but fixed $x_{0} \in[0, T]$. Therefore we should establish the estimate: there exist $\widehat{\tau}>1$ and $N_{0}>1$ such that for any $\tau>\widehat{\tau}$ and $N>N_{0}$, there exists $s_{0}(\tau, N)$ such that

$$
\begin{array}{r}
N \int_{\Omega_{1 / N^{2}}}\left(\frac{1}{s \varphi} \sum_{j, k=1}^{2}\left|\partial_{x_{j}} \partial_{x_{k}} \mathbf{u}\right|^{2}+s \varphi\left|\nabla_{x^{\prime}} \mathbf{u}\right|^{2}+s^{3} \varphi^{3}|\mathbf{u}|^{2}\right) \mathrm{e}^{2 s \varphi} \mathrm{d} x^{\prime} \leq C_{0}\left(\left\|\operatorname{rot} \mathbf{u} \mathrm{e}^{s \varphi}\right\|_{H^{1}\left(\Omega_{1 / N^{2}}\right)}^{2}+\left\|\operatorname{div} \mathbf{u} \mathrm{e}^{s \varphi}\right\|_{H^{1}\left(\Omega_{1 / N^{2}}\right)}^{2}\right), \\
\forall \mathbf{u} \in\left(H_{0}^{1}\left(\Omega_{1 / N^{2}}\right)\right)^{2}, \quad \forall s \geq s_{0}(\tau, N), \quad \operatorname{supp} \mathbf{u} \subset B_{\delta} \cap \Omega_{1 / N^{2}}, \quad(1)
\end{array}
$$

where the constant $C_{0}$ is independent of $N$. Recall that $\Omega_{1 / N^{2}}=\left\{x^{\prime} \in \Omega\right.$; dist $\left.\left(x^{\prime}, \partial \Omega\right) \leq \frac{1}{N^{2}}\right\}$.

First we choose $N_{0}>0$ sufficiently large such that

$$
\nabla_{x^{\prime}} \psi(x) \neq 0, \quad \forall x^{\prime} \in \Omega_{1 / N^{2}}, \forall x_{0} \in(0, T) .
$$

The existence of such $N_{0}$ follows from (2.6).

Denote $\operatorname{rot} \mathbf{u} \equiv \frac{\partial u_{2}}{\partial x_{1}}-\frac{\partial u_{1}}{\partial x_{2}}=\mathbf{y}$ and $\operatorname{div} \mathbf{u} \equiv \mathbf{w}$. Let $\operatorname{rot}^{*} v=\left(\frac{\partial v}{\partial x_{2}},-\frac{\partial v}{\partial x_{1}}\right)$. Using a formula $\operatorname{rot}^{*} \operatorname{rot}=-\Delta_{x^{\prime}}+$ $\nabla_{x^{\prime}}$ div, we obtain

$$
-\Delta_{x^{\prime}} \mathbf{u}=-\operatorname{rot}^{*} \mathbf{y}-\nabla_{x^{\prime}} \mathbf{w} \quad \text { in } \Omega_{1 / N^{2}},\left.\quad \mathbf{u}\right|_{\partial \Omega_{1 / N^{2}}}=0 .
$$

The function $\widetilde{\mathbf{u}}=\mathbf{u e}^{s \varphi}$ satisfies the equation

$$
L_{1} \widetilde{\mathbf{u}}+L_{2} \widetilde{\mathbf{u}}=\mathbf{q}_{s} \text { in } \Omega_{1 / N^{2}},\left.\widetilde{\mathbf{u}}\right|_{\partial \Omega_{1 / N^{2}}}=0
$$

where $L_{1} \widetilde{\mathbf{u}}=-\Delta_{x^{\prime}} \widetilde{\mathbf{u}}-s^{2}\left|\nabla_{x^{\prime}} \varphi\right|^{2} \widetilde{\mathbf{u}}, L_{2} \widetilde{\mathbf{u}}=2 s \sum_{k=1}^{2}\left(\partial_{x_{k}} \widetilde{\mathbf{u}}\right) \varphi_{x_{k}}+s\left(\Delta_{x^{\prime}} \varphi\right) \widetilde{\mathbf{u}}$ and $\mathbf{q}_{s}=\left(-\operatorname{rot}^{*} \mathbf{y}-\nabla_{x^{\prime}} \mathbf{w}\right) \mathrm{e}^{s \varphi}$. Taking the $L^{2}$ norms of the right and the left hand sides of equation (2), we obtain

$$
\left\|L_{1} \widetilde{\mathbf{u}}\right\|_{\left(L^{2}\left(\Omega_{1 / N^{2}}\right)\right)^{2}}^{2}+\left\|L_{2} \widetilde{\mathbf{u}}\right\|_{\left(L^{2}\left(\Omega_{1 / N^{2}}\right)\right)^{2}}^{2}+2\left(L_{1} \widetilde{\mathbf{u}}, L_{2} \widetilde{\mathbf{u}}\right)_{\left(L^{2}\left(\Omega_{1 / N^{2}}\right)\right)^{2}}=\left\|\mathbf{q}_{s}\right\|_{\left(L^{2}\left(\Omega_{1 / N^{2}} v\right)^{2}\right.}^{2} .
$$

Therefore we can obtain the formula

$$
\begin{aligned}
\left(L_{1} \widetilde{\mathbf{u}}, L_{2} \widetilde{\mathbf{u}}\right)_{\left(L^{2}\left(\Omega_{1 / N^{2}}\right)\right)^{2}=} \int_{\Omega_{\Omega_{1 / N}}} & \left(2 s \sum_{k, j=1}^{2}\left(\partial_{x_{j}} \widetilde{\mathbf{u}}\right)\left(\partial_{x_{k}} \widetilde{\mathbf{u}}\right) \varphi_{x_{j} x_{k}}+s^{3}\left(\operatorname{div}\left(\left|\nabla_{x^{\prime}} \varphi\right|^{2} \nabla_{x^{\prime}} \varphi\right)\right.\right. \\
& \left.\left.\quad-\left|\nabla_{x^{\prime}} \varphi\right|^{2} \Delta_{x^{\prime}} \varphi\right)|\widetilde{\mathbf{u}}|^{2}-\frac{s}{2} \sum_{j=1}^{2} \frac{\partial^{2} \Delta_{x^{\prime}} \varphi}{\partial x_{j}^{2}}|\widetilde{\mathbf{u}}|^{2}\right) \mathrm{d} x^{\prime}-s \int_{\partial \Omega_{\Omega_{1 / N^{2}}}}\left|\frac{\partial \widetilde{\mathbf{u}}}{\partial \vec{n}}\right|^{2}\left(\nabla_{x^{\prime}} \varphi, \vec{n}\right) \mathrm{d} \sigma .
\end{aligned}
$$


By (2.6), the last integral in (3) is nonnegative. Denote $\psi_{1}(x)=\psi(x)-\widehat{\varepsilon} \ell_{1}(x)$. Then

$$
\begin{aligned}
& \operatorname{div}\left(\left|\nabla_{x^{\prime}} \varphi\right|^{2} \nabla_{x^{\prime}} \varphi\right)-\left|\nabla_{x^{\prime}} \varphi\right|^{2} \Delta_{x^{\prime}} \varphi=2 \sum_{k, j=1}^{2} \varphi_{x_{k}} \varphi_{x_{j}} \varphi_{x_{k} x_{j}} \\
& =2 \varphi^{3} \sum_{k, j=1}^{2} \tau^{4}\left(\partial_{x_{k}} \psi_{1}+2 N \ell_{1} \partial_{x_{k}} \ell_{1}\right)^{2}\left(\partial_{x_{j}} \psi_{1}+2 N \ell_{1} \partial_{x_{j}} \ell_{1}\right)^{2} \\
& +\tau^{3}\left(\partial_{x_{k}} \psi_{1}+2 N \ell_{1} \partial_{x_{k}} \ell_{1}\right)\left(\partial_{x_{j}} \psi_{1}+2 N \ell_{1} \partial_{x_{j}} \ell_{1}\right)\left(\partial_{x_{j}} \partial_{x_{k}} \psi_{1}+2 N \partial_{x_{k}} \ell_{1} \partial_{x_{j}} \ell_{1}+2 N \ell_{1} \partial_{x_{k}} \partial_{x_{j}} \ell_{1}\right)
\end{aligned}
$$

Since $\left(\nabla_{x^{\prime}} \psi_{1}, \nabla_{x^{\prime}} \ell_{1}\right)>0$ on $\partial \Omega$, there exists a constant $C_{1}>0$ which is independent of $N, \tau, s$ such that

$$
\operatorname{div}\left(\left|\nabla_{x^{\prime}} \varphi\right|^{2} \nabla_{x^{\prime}} \varphi\right)-\left|\nabla_{x^{\prime}} \varphi\right|^{2} \Delta_{x^{\prime}} \varphi \geq 2 \varphi^{3} \tau^{4}\left|\nabla_{x^{\prime}} \psi_{1}\right|^{4}+C_{1} N \tau^{3} \varphi^{3}+\varphi^{2} O\left(\tau^{3}\right)
$$

On the other hand, by the definition of $\widetilde{\psi}=\psi-\widehat{\varepsilon} \ell_{1}+N \ell_{1}^{2}=\psi_{1}+N \ell_{1}^{2}$,

$$
\begin{aligned}
& \sum_{k, j=1}^{2}\left(\partial_{x_{j}} \widetilde{\mathbf{u}}\right)\left(\partial_{x_{k}} \widetilde{\mathbf{u}}\right) \varphi_{x_{j} x_{k}}=\tau^{2}\left(\nabla_{x^{\prime}} \widetilde{\mathbf{u}}, \nabla_{x^{\prime}} \widetilde{\psi}\right)^{2} \varphi \\
& \quad+\tau \sum_{k, j=1}^{2}\left(\partial_{x_{j}} \widetilde{\mathbf{u}}\right)\left(\partial_{x_{k}} \widetilde{\mathbf{u}}\right)\left(\partial_{x_{j}} \partial_{x_{k}} \psi_{1}+2 N \ell_{1} \partial_{x_{j}} \partial_{x_{k}} \ell_{1}\right) \varphi+2 N \tau\left(\nabla_{x^{\prime}} \widetilde{\mathbf{u}}, \nabla_{x^{\prime}} \ell_{1}\right)^{2} \varphi
\end{aligned}
$$

Note that there exists a constant $C_{2}>0$, independent of $N$, such that

$$
\left\|N \ell_{1} \partial_{x_{i} x_{j}}^{2} \ell_{1}\right\|_{C^{0}\left(\overline{\Omega_{1 / N^{2}}}\right)} \leq C_{2} / N
$$

By (3)-(6), we obtain

$$
\begin{array}{r}
\left\|L_{1} \widetilde{\mathbf{u}}\right\|_{\left(L^{2}\left(\Omega_{1 / N^{2}}\right)\right)^{2}}^{2}+\left\|L_{2} \widetilde{\mathbf{u}}\right\|_{\left(L^{2}\left(\Omega_{1 / N^{2}}\right)\right)^{2}}^{2}+\int_{\Omega_{1 / N^{2}}}\left(2 \varphi^{3} \tau^{4}\left|\nabla_{x^{\prime}} \psi_{1}\right|^{4}+C_{1} N \tau^{3} \varphi^{3}\right)|\widetilde{\mathbf{u}}|^{2} \mathrm{~d} x^{\prime} \\
-s \tau C_{3} \int_{\Omega_{1 / N^{2}}} \varphi\left|\nabla_{x^{\prime}} \widetilde{\mathbf{u}}\right|^{2} \mathrm{~d} x^{\prime} \leq\left\|\mathbf{q}_{s}\right\|_{\left(L^{2}\left(\Omega_{1 / N^{2}}\right)\right)^{2}}^{2}
\end{array}
$$

Multiplying equation (2) by $s N \varphi \widetilde{\mathbf{u}}$ and integrating by parts, we obtain

$$
\int_{\Omega_{1 / N^{2}}}\left(s N \varphi\left|\nabla_{x^{\prime}} \widetilde{\mathbf{u}}\right|^{2}+s^{2} N\left(\Delta_{x^{\prime}} \varphi\right) \varphi|\widetilde{\mathbf{u}}|^{2}-s^{3} \varphi^{3}\left|\nabla_{x^{\prime}} \varphi\right|^{2}|\widetilde{\mathbf{u}}|^{2}-\frac{s N}{2} \operatorname{div} \varphi|\widetilde{\mathbf{u}}|^{2}\right) \mathrm{d} x^{\prime}=\int_{\Omega_{1 / N^{2}}} \mathbf{q}_{s} s N \varphi \widetilde{\mathbf{u}} \mathrm{d} x^{\prime} .
$$

Next we note that

$$
\Delta_{x^{\prime}} \varphi=\left(\left|\nabla_{x^{\prime}} \widetilde{\psi}\right|^{2} \tau^{2}+\tau \Delta_{x^{\prime}} \psi_{1}+2 \tau N\left|\nabla_{x^{\prime}} \ell_{1}\right|^{2}+2 \tau N \ell_{1} \Delta_{x^{\prime}} \ell_{1}\right) \varphi \geq C_{4} \tau N \varphi
$$

This inequality and (8) imply

$$
\int_{\Omega_{1 / N^{2}}}\left\{s N \varphi\left|\nabla_{x^{\prime}} \widetilde{\mathbf{u}}\right|^{2}+\frac{1}{2} s^{2} N\left(\Delta_{x^{\prime}} \varphi\right) \varphi|\widetilde{\mathbf{u}}|^{2}-s^{3} \varphi^{3}\left|\nabla_{x^{\prime}} \varphi\right|^{2}|\widetilde{\mathbf{u}}|^{2}\right\} \mathrm{d} x^{\prime} \leq C_{4}\left\|\mathbf{q}_{s}\right\|_{\left(L ^ { 2 } \left(\Omega_{\left.\left.1 / N^{2}\right)\right)^{2}}\right.\right.}^{2}
$$


By (7) and (9), we obtain

$$
\begin{aligned}
\left\|L_{1} \widetilde{\mathbf{u}}\right\|_{\left(L^{2}\left(\Omega_{1 / N^{2}}\right)\right)^{2}}^{2}+\left\|L_{2} \widetilde{\mathbf{u}}\right\|_{\left(L^{2}\left(\Omega_{1 / N^{2}}\right)\right)^{2}}^{2}+\int_{\Omega_{1 / N^{2}}}\left(\frac{1}{2} \varphi^{3} \tau^{4}\left|\nabla_{x^{\prime}} \psi_{1}\right|^{4}+C_{1} N \tau^{3} \varphi^{3}\right)|\widetilde{\mathbf{u}}|^{2} \mathrm{~d} x^{\prime} & \\
& +s N \int_{\Omega_{1 / N^{2}}} \varphi\left|\nabla_{x^{\prime}} \widetilde{\mathbf{u}}\right|^{2} \mathrm{~d} x^{\prime} \leq C_{5}\left\|\mathbf{q}_{s}\right\|_{\left(L^{2}\left(\Omega_{1 / N^{2}}\right)\right)^{2}}^{2}
\end{aligned}
$$

Let $\widetilde{\mathbf{u}}=\widetilde{\mathbf{u}}_{1}+\widetilde{\mathbf{u}}_{2}$ where the functions $\widetilde{\mathbf{u}}_{j}$ are solutions to the boundary value problems

$$
-\Delta_{x^{\prime}} \widetilde{\mathbf{u}}_{1}=L_{1} \widetilde{\mathbf{u}} \quad \text { in } \quad \Omega_{1 / N_{0}^{2}},\left.\quad \widetilde{\mathbf{u}}_{1}\right|_{\partial \Omega_{1 / N_{0}^{2}}}=0, \quad-\Delta_{x^{\prime}} \widetilde{\mathbf{u}}_{2}=s^{2}\left|\nabla_{x^{\prime}} \varphi\right|^{2} \widetilde{\mathbf{u}} \text { in } \Omega_{1 / N_{0}^{2}},\left.\widetilde{\mathbf{u}}_{2}\right|_{\partial \Omega_{1 / N_{0}^{2}}}=0 .
$$

By means of a standard a priori estimate for the Laplace operator, we have

$$
\begin{gathered}
\left\|\widetilde{\mathbf{u}}_{1}\right\|_{\left(H^{2}\left(\Omega_{1 / N^{2}}\right)\right)^{2}} \leq C_{6}\left\|L_{1} \widetilde{\mathbf{u}}\right\|_{\left(L^{2}\left(\Omega_{1 / N^{2}}\right)\right)^{2}}, \\
\frac{\sqrt{N}}{\sqrt{s}}\left\|\widetilde{\mathbf{u}}_{2}\right\|_{\left(H^{2}\left(\Omega_{1 / N^{2}}\right)\right)^{2}} \leq C \sqrt{N}\left\|s^{\frac{3}{2}}\left|\nabla_{x^{\prime}} \varphi\right|^{2} \widetilde{\mathbf{u}}\right\|_{\left(L^{2}\left(\Omega_{1 / N^{2}}\right)\right)^{2}},
\end{gathered}
$$

where the constants $C_{6}$ and $C_{7}$ are independent of $N$. Taking $s_{0}(\tau, N) \geq N$, we obtain (1) from (9)-(12). The proof of Proposition 5.1 is finished.

\section{Appendix II. Proof of estimate (5.28)}

We prove (5.28) for a more general hyperbolic operator. Denote $y=\left(y_{0}, y^{\prime}\right)=\left(y_{0}, y_{1}, \ldots, y_{n}\right), \xi=\left(\xi_{0}, \xi^{\prime}\right)=$ $\left(\xi_{0}, \xi_{1}, \ldots, \xi_{n}\right)$ and $\mathcal{G}_{N}=\mathbb{R}^{n} \times\left[0, \frac{1}{N^{2}}\right]$.

Let a function $w \in H^{1}\left(\mathcal{G}_{N}\right)$ satisfy the equations:

$$
\begin{aligned}
R\left(y^{\prime}, D\right) w & =\frac{\partial^{2} w}{\partial y_{0}^{2}}-\sum_{j, k=1}^{n} \frac{\partial}{\partial y_{j}}\left(a_{j k}\left(y^{\prime}\right) \frac{\partial w}{\partial y_{k}}\right)+\sum_{j=0}^{n} b_{j}\left(y^{\prime}\right) \frac{\partial w}{\partial y_{j}}+c\left(y^{\prime}\right) w=g \text { in } \mathcal{G}_{N}, \\
\left.w\right|_{y_{n}=\frac{1}{N^{2}}} & =\left.\frac{\partial w}{\partial y_{n}}\right|_{y_{n}=\frac{1}{N^{2}}}=0, \quad \operatorname{supp} w \subset B_{\delta}\left(x^{*}\right)
\end{aligned}
$$

where $x^{*}$ is an arbitrary point on $\partial \mathcal{G}_{N}$ and $B_{\delta}\left(x^{*}\right)$ is a ball of radius $\delta$ centered at $x^{*}$.

We assume that the coefficients of the linear operator $R$ satisfy the conditions

$$
a_{j k} \in C^{1}\left(\overline{\mathcal{G}_{N}}\right), a_{j k}=a_{k j}, \quad 1 \leq j, k \leq n, \quad b_{\ell} \in L^{\infty}\left(\mathcal{G}_{N}\right), \quad 0 \leq \ell \leq n, \quad c \in L^{\infty}\left(\mathcal{G}_{N}\right)
$$

and the uniform ellipticity: there exists $\delta>0$ such that

$$
a\left(y^{\prime}, \xi, \xi\right) \equiv \sum_{j, k=1}^{n} a_{j k}\left(y^{\prime}\right) \xi_{j} \xi_{k} \geq \delta|\xi|^{2}, \quad \forall \xi \in \mathbb{R}^{n+1}, \quad \forall y \in \overline{\mathcal{G}_{N}}
$$

By $R\left(y^{\prime}, \xi\right)$, we denote the principal symbol of the operator $R$ :

$$
R\left(y^{\prime}, \xi\right)=-\xi_{0}^{2}+\sum_{j, k=1}^{n} a_{j k}\left(y^{\prime}\right) \xi_{j} \xi_{k},
$$


and by $\widetilde{R}\left(y^{\prime}, \xi^{1}, \xi^{2}\right)$ the quadratic form

$$
\widetilde{R}\left(y^{\prime}, \xi^{1}, \xi^{2}\right)=\xi_{0}^{1} \xi_{0}^{2}-\sum_{j, k=1}^{n} a_{j k}\left(y^{\prime}\right) \xi_{j}^{1} \xi_{k}^{2}
$$

with $\xi^{1}=\left(\xi_{0}^{1}, \ldots, \xi_{n}^{1}\right)$ and $\xi^{2}=\left(\xi_{0}^{2}, \ldots, \xi_{n}^{2}\right)$. Following [15], we introduce the notations:

$$
R^{(j)}\left(y^{\prime}, \xi\right)=\frac{\partial R\left(y^{\prime}, \xi\right)}{\partial \xi_{j}}, \quad R^{(j, k)}\left(y^{\prime}, \xi\right)=\frac{\partial^{2} R\left(y^{\prime}, \xi\right)}{\partial \xi_{j} \partial \xi_{k}}, \quad R_{(j)}\left(y^{\prime}, \xi\right)=\frac{\partial R\left(y^{\prime}, \xi\right)}{\partial y_{j}} .
$$

We assume that there exists a function $\psi_{1} \in C^{2}\left(\overline{\mathcal{G}_{N}}\right)$ such that

$$
\left\{R,\left\{R, \psi_{1}\right\}\right\}(y, \xi)>0
$$

if $(y, \xi) \in\left(\overline{\mathcal{G}_{N} \backslash B_{\delta}\left(x^{*}\right)}\right) \times\left(\mathbb{R}^{n+1} \backslash\{0\}\right)$ satisfies

$$
R\left(y^{\prime}, \xi\right)=\left\langle\nabla_{\xi} R\left(y^{\prime}, \xi\right), \nabla \psi_{1}(y)\right\rangle=0,
$$

and

$$
\left\{R\left(y^{\prime}, \xi-i s \nabla \psi_{1}(y)\right), R\left(y^{\prime}, \xi+i s \nabla \psi_{1}(y)\right)\right\} / 2 i s>0
$$

if $(y, \xi, s) \in\left(\overline{\mathcal{G}_{N} \backslash B_{\delta}\left(x^{*}\right)}\right) \times\left(\mathbb{R}^{n+1} \backslash\{0\}\right) \times(\mathbb{R} \backslash\{0\})$ satisfies

$$
\begin{aligned}
R\left(y^{\prime}, \xi+i s \nabla \psi_{1}(y)\right)= & \left\langle\nabla_{\xi} R\left(y^{\prime}, \xi+i s \nabla \psi_{1}(y)\right), \nabla \psi_{1}(y)\right\rangle=0, \\
& R\left(y, \nabla \psi_{1}\right)<0 .
\end{aligned}
$$

Using the function $\psi_{1}$ and following [15], we construct the function $\phi$ by

$$
\phi(y)=\mathrm{e}^{\tau \psi_{1}(y)}, \quad \tau>1 .
$$

It is known (see e.g., Th. 8.6.2, p. 205 [15]) that provided that the parameter $\tau$ is sufficiently large,

$$
\{R,\{R, \phi\}\}(y, \xi)>0
$$

if $(y, \xi) \in\left(\overline{\mathcal{G}_{N} \backslash B_{\delta}\left(x^{*}\right)}\right) \times\left(\mathbb{R}^{n+1} \backslash\{0\}\right)$ satisfies

$$
R\left(y^{\prime}, \xi\right)=0,
$$

and

$$
\left\{R\left(y^{\prime}, \xi-i s \nabla \phi(y)\right), R\left(y^{\prime}, \xi+i s \nabla \phi(y)\right)\right\} / 2 i s>0
$$

if $(y, \xi, s) \in\left(\overline{\mathcal{G}_{N} \backslash B_{\delta}\left(x^{*}\right)}\right) \times\left(\mathbb{R}^{n+1} \backslash\{0\}\right) \times(\mathbb{R} \backslash\{0\})$ satisfies

$$
R\left(y^{\prime}, \xi+i s \nabla \phi(x)\right)=0 .
$$

Now we fix the parameter $\tau$ such that inequalities (8) and (9) hold true. Let $\ell_{1} \in C^{2}\left(\mathcal{G}_{N}\right)$ be a function such that $\left.\ell_{1}\right|_{y_{n}=0}=0$. Let $\widetilde{\psi}(y)=\psi_{1}(y)+N \ell_{1}^{2}(y)$ and $\varphi=\mathrm{e}^{\tau \widetilde{\psi}}$. Since $\varphi(y)=\phi(y) \mathrm{e}^{\tau N \ell_{1}^{2}(y)}$, using $\left.\ell_{1}\right|_{y_{n}=0}=0$, we have

$$
\varphi \rightarrow \phi \quad \text { in } C^{1}\left(\overline{\mathcal{G}_{N}}\right) \text { as } N \rightarrow+\infty .
$$


Moreover

$$
\begin{aligned}
\left\{R\left(y^{\prime}, \xi-i s \nabla \varphi(y)\right),\right. & \left.R\left(y^{\prime}, \xi+i s \nabla \varphi(y)\right)\right\} / 2 i s \\
-2 N \tau & \sum_{j, k=1}^{n}\left(\partial_{y_{j}} \ell_{1}(y)\right)\left(\partial_{y_{k}} \ell_{1}(y)\right)\left(R^{(j)}\left(y^{\prime}, \xi\right) R^{(k)}\left(y^{\prime}, \xi\right)+s^{2} R^{(j)}\left(y^{\prime}, \nabla \varphi\right) R^{(k)}\left(y^{\prime}, \nabla \varphi\right)\right) \\
& \longrightarrow\left\{R\left(y^{\prime}, \xi-i s \nabla \phi(y)\right), R\left(y^{\prime}, \xi+i s \nabla \phi(y)\right)\right\} / 2 i s \text { in } C\left(\mathcal{G}_{N} \times \mathbb{S}^{n}\right) \quad \text { as } \quad N \rightarrow+\infty
\end{aligned}
$$

Here we set $\mathbb{S}^{n}=\left\{\xi \in \mathbb{R}^{n+1} ;|\xi|=1\right\}$. By (8)-(11), there exists $N_{0}>0$ such that for any $N>N_{0}$, the following inequalities hold true:

$$
\{R,\{R, \varphi\}\}(y, \xi)>0
$$

if $(y, \xi) \in\left(\overline{\mathcal{G}_{N} \backslash B_{\delta}\left(x^{*}\right)}\right) \times\left(\mathbb{R}^{n+1} \backslash\{0\}\right)$ satisfies $R(y, \xi)=0$, and

$$
\left\{R\left(y^{\prime}, \xi-i s \nabla \varphi(y)\right), R\left(y^{\prime}, \xi+i s \nabla \varphi(y)\right)\right\} / 2 i s>C_{1}\left(|\xi|^{2}+N s^{2}\right)
$$

if $(y, \xi, s) \in\left(\overline{\mathcal{G}_{N} \backslash B_{\delta}\left(x^{*}\right)}\right) \times\left(\mathbb{R}^{n+1} \backslash\{0\}\right) \times(\mathbb{R} \backslash\{0\})$ satisfies $R\left(y^{\prime}, \xi+i s \nabla \varphi(y)\right)=0$, where the constant $C_{1}>0$ is independent of $\xi, s, N$.

Denote $\widetilde{w}(y)=w(y) e^{s \varphi}$. By $(11)$, the following equality holds:

$$
\mathrm{e}^{s \phi} R\left(y^{\prime}, D\right)\left(\mathrm{e}^{-s \varphi} \widetilde{w}\right)=g \mathrm{e}^{s \varphi} \quad \text { in } \mathcal{G}_{N}
$$

The short calculations give the equation

$$
L_{2, \varphi} \widetilde{w}+L_{1, \varphi} \widetilde{w}=g_{s} \text { in } \mathcal{G}_{N},
$$

where

$$
\begin{aligned}
& L_{1, \varphi} \widetilde{w}=-\sum_{j=0}^{n} s \varphi_{y_{j}} R^{(j)}\left(y^{\prime}, \nabla \widetilde{w}\right), \quad L_{2, \varphi} \widetilde{w}=R \widetilde{w}+s^{2} R\left(y^{\prime}, \nabla \varphi\right) \widetilde{w} \\
& g_{s}(y)=g e^{s \varphi}+\widetilde{w} R \varphi
\end{aligned}
$$

Taking the $L_{2}$-norms of the both sides of (15), we obtain

$$
\left\|g_{s}\right\|_{L^{2}\left(\mathcal{G}_{N}\right)}^{2}=\left\|L_{2, \varphi} \widetilde{w}\right\|_{L^{2}\left(\mathcal{G}_{N}\right)}^{2}+\left\|L_{1, \varphi} \widetilde{w}\right\|_{L^{2}\left(\mathcal{G}_{N}\right)}^{2}+2\left(L_{1, \varphi} \widetilde{w}, L_{2, \varphi} \widetilde{w}\right)_{L^{2}\left(\mathcal{G}_{N}\right)} .
$$

Denote

$$
\begin{aligned}
G_{\phi}(y, s, \widetilde{w})=\{R,\{R, \phi\}\}\left(y^{\prime}, \nabla \widetilde{w}\right)+s^{2} \sum_{j, k=0}^{n} R_{(k)}\left(y^{\prime}, \nabla \phi\right) R^{(j)}\left(y^{\prime}, \nabla \phi\right) \widetilde{w}^{2} \\
+s^{2} \sum_{j, k=0}^{n} \phi_{y_{j} y_{k}} R^{(j)}\left(y^{\prime}, \nabla \phi\right) R^{(k)}\left(y^{\prime}, \nabla \phi\right) \widetilde{w}^{2}
\end{aligned}
$$

and $G_{\varphi}(y, s, \widetilde{w})$ is defined similarly. 
Let us transform the last term at the right side of (17). In [18], one can find the following identity:

$$
\begin{aligned}
\left(L_{1, \varphi} \widetilde{w}, L_{2, \varphi} \widetilde{w}\right)_{L^{2}\left(\mathcal{G}_{N}\right)}= & \int_{\partial \mathcal{G}_{N}} \widetilde{R}\left(y^{\prime}, \vec{n}, \nabla \widetilde{w}\right) L_{1, \varphi} \widetilde{w} \mathrm{~d} \Sigma+s \int_{\partial \mathcal{G}_{N}} \widetilde{R}\left(y^{\prime}, \nabla \varphi, \vec{n}\right) R\left(y^{\prime}, \nabla \widetilde{w}\right) \mathrm{d} \Sigma \\
& -s^{3} \int_{\partial \mathcal{G}_{N}} R\left(y^{\prime}, \nabla \varphi\right) \widetilde{R}\left(y^{\prime}, \vec{n}, \nabla \varphi\right) \widetilde{w}^{2} \mathrm{~d} \Sigma+\int_{\mathcal{G}_{N}} s G_{\varphi}(y, s, \widetilde{w}) \mathrm{d} x \\
& +\int_{\mathcal{G}_{N}} \frac{s}{2}\left(\sum_{j, k=0}^{n} R_{(k)}^{(k)}\left(y^{\prime}, \nabla \widetilde{w}\right) \varphi_{y_{j}} R^{(j)}\left(y^{\prime}, \nabla \widetilde{w}\right)-\theta\left(R\left(y^{\prime}, \nabla \widetilde{w}\right)-s^{2} R\left(y^{\prime}, \nabla \varphi\right) \widetilde{w}^{2}\right)\right) \mathrm{d} y
\end{aligned}
$$

where $\vec{n}$ is the unit outward normal vector to $\partial \mathcal{G}_{N}$ and

$$
\theta(y)=\sum_{l, m=0}^{n}\left(\varphi_{y_{l} y_{m}} R^{(l, m)}\left(y^{\prime}, \nabla \widetilde{w}\right)+\varphi_{y_{l}} R_{(m)}^{(l, m)}\left(y^{\prime}, \nabla \widetilde{w}\right)\right)
$$

Now we need the following Lemma proved in [18].

Lemma 1. Let $w \in H^{1}\left(\mathcal{G}_{N}\right)$ be a solution to (1) and (2).

$$
\begin{aligned}
s \int_{\mathcal{G}_{N}}\left(|\nabla \widetilde{w}|^{2}+\right. & \left.s^{2} \widetilde{w}^{2}\right) \mathrm{d} y \leq C_{2} \int_{\mathcal{G}_{N}} s G_{\phi}(y, s, \widetilde{w}) \mathrm{d} y \\
& +C_{3}\left(\frac{1}{s}\left\|L_{2, \phi} \widetilde{w}\right\|_{L^{2}\left(\mathcal{G}_{N}\right)}^{2}+\frac{1}{s}\left\|L_{1, \phi} \widetilde{w}\right\|_{L^{2}\left(\mathcal{G}_{N}\right)}^{2}+s\|\widetilde{w}\|_{L^{2}\left(\partial \mathcal{G}_{N}\right)}\left\|\partial_{y_{n}} \widetilde{w}\right\|_{L^{2}\left(\partial \mathcal{G}_{N}\right)}\right), \quad \forall s \geq s_{0}(\tau)
\end{aligned}
$$

where the constants $C_{2}$ and $C_{3}$ are independent of $s, N$.

We claim :

$$
\begin{array}{r}
\left|\int_{\mathcal{G}_{N}} \frac{s}{2}\left(\sum_{j, k=0}^{n} R_{(k)}^{(k)}\left(y^{\prime}, \nabla \widetilde{w}\right) \varphi_{y_{j}} R^{(j)}\left(y^{\prime}, \nabla \widetilde{w}\right)-\theta\left\{R\left(y^{\prime}, \nabla \widetilde{w}\right)-s^{2} R\left(y^{\prime}, \nabla \varphi\right) \widetilde{w}^{2}\right\}\right) \mathrm{d} y\right| \\
\leq\left|\frac{s}{2} \int_{\mathcal{G}_{N}} \sum_{j, k=0}^{n} R_{(k)}^{(k)}\left(y^{\prime}, \nabla \widetilde{w}\right) \varphi_{x_{j}} R^{(j)}\left(y^{\prime}, \nabla \widetilde{w}\right) d y\right|+\left|s \int_{\mathcal{G}_{N}} \theta\left(R\left(y^{\prime}, \nabla \widetilde{w}\right)-s^{2} R\left(y^{\prime}, \nabla \varphi\right) \widetilde{w}^{2}\right) \mathrm{d} y\right| \\
\leq \frac{\varepsilon s}{2} \int_{\mathcal{G}_{N}}\left(|\nabla \widetilde{w}|^{2}+s^{2} \widetilde{w}^{2}\right) \mathrm{d} y+C_{4}\left(\frac{1}{s \varepsilon}\left\|L_{1, \varphi} \widetilde{w}\right\|_{L^{2}\left(\mathcal{G}_{N}\right)}^{2}+\frac{1}{s \varepsilon}\left\|L_{2, \varphi} \widetilde{w}\right\|_{L^{2}\left(\mathcal{G}_{N}\right)}^{2}+s\|\widetilde{w}\|_{L^{2}\left(\partial \mathcal{G}_{N}\right)}\left\|\partial_{y_{n}} \widetilde{w}\right\|_{L^{2}\left(\partial \mathcal{G}_{N}\right)}\right) .
\end{array}
$$

In fact, by the Cauchy-Bunyakovskii inequality,

$$
\left|\int_{\mathcal{G}_{N}} s \sum_{j, k=0}^{n} R_{(k)}^{(k)}\left(y^{\prime}, \nabla \widetilde{w}\right) \varphi_{y_{j}} R^{(j)}\left(y^{\prime}, \nabla \widetilde{w}\right) \mathrm{d} y\right| \leq \frac{\varepsilon s}{4}\|\widetilde{w}\|_{H^{1}\left(\mathcal{G}_{N}\right)}^{2}+\frac{C_{5}}{s \varepsilon}\left\|L_{1, \varphi} \widetilde{w}\right\|_{L^{2}\left(\mathcal{G}_{N}\right)}^{2}
$$


Since the function $\theta$ is continuous, there exists $\theta_{\varepsilon} \in C^{2}\left(\overline{\mathcal{G}_{N}}\right)$ such that $\left\|\theta-\theta_{\varepsilon}\right\|_{C\left(\overline{\mathcal{G}_{N}}\right)} \leq \frac{\varepsilon}{8}$. Taking the scalar product in $L^{2}\left(\mathcal{G}_{N}\right)$ of the functions $\theta_{\varepsilon} \widetilde{w}$ and $L_{2, \varphi} \widetilde{w}$, we obtain the equality

$$
\begin{aligned}
\int_{\mathcal{G}_{N}} \theta_{\varepsilon}\left(s R\left(y^{\prime}, \nabla \widetilde{w}\right)-s^{3} R\left(y^{\prime}, \nabla \varphi\right) \widetilde{w}^{2}\right) \mathrm{d} y & =-s \int_{\mathcal{G}_{N}}\left(L_{2, \varphi} \widetilde{w}\right) \theta_{\varepsilon} \widetilde{w} \mathrm{~d} y \\
& +s \int_{\mathcal{G}_{N}} \sum_{j, k=1}^{n}\left(\frac{\partial a_{j k}}{\partial y_{j}} \frac{\partial \widetilde{w}}{\partial y_{k}} \theta_{\varepsilon} \widetilde{w}-\widetilde{R}\left(y^{\prime}, \nabla \widetilde{w}, \nabla \theta_{\varepsilon}\right) \widetilde{w}\right) \mathrm{d} y+\int_{\partial \mathcal{G}_{N}} a(y, \vec{n}, \nabla \widetilde{w}) \theta_{\varepsilon} \widetilde{w} \mathrm{~d} \Sigma .
\end{aligned}
$$

Thus

$$
\begin{gathered}
\left|\int_{\mathcal{G}_{N}} \theta\left(s R\left(y^{\prime}, \nabla \widetilde{w}\right)-s^{3} R\left(y^{\prime}, \nabla \varphi\right) \widetilde{w}^{2}\right) \mathrm{d} y\right| \\
\leq\left|\int_{\mathcal{G}_{N}}\left(\theta-\theta_{\varepsilon}\right)\left(s R\left(y^{\prime}, \nabla \widetilde{w}\right)-s^{3} R\left(y^{\prime}, \nabla \varphi\right) \widetilde{w}^{2}\right) \mathrm{d} y\right|+\left|\int_{\mathcal{G}_{N}} \theta_{\varepsilon}\left(s R\left(x^{\prime}, \nabla \widetilde{w}\right)-s^{3} R\left(x^{\prime}, \nabla \varphi\right) \widetilde{w}^{2}\right) \mathrm{d} y\right| \\
\leq \frac{\varepsilon s}{4} \int_{\mathcal{G}_{N}}\left(|\nabla \widetilde{w}|^{2}+s^{2} \widetilde{w}^{2}\right) \mathrm{d} y+C_{6}\left(\frac{1}{s}\left\|L_{1, \varphi} \widetilde{w}\right\|_{L^{2}\left(\mathcal{G}_{N}\right)}^{2}+\frac{1}{s}\left\|L_{2, \varphi} \widetilde{w}\right\|_{L^{2}\left(\mathcal{G}_{N}\right)}^{2}+s\|\widetilde{w}\|_{L^{2}\left(\partial \mathcal{G}_{N}\right)}\left\|\partial_{y_{n}} \widetilde{w}\right\|_{L^{2}\left(\partial \mathcal{G}_{N}\right)}\right) .
\end{gathered}
$$

Inequalities (22) and (23) imply (21).

By Lemma 1, we have

$$
\begin{aligned}
& \quad s \int_{\mathcal{G}_{N}}\left(|\nabla \widetilde{w}|^{2}+s^{2} \widetilde{w}^{2}\right) \mathrm{d} y+\int_{\mathcal{G}_{N}} 4 N \tau \sum_{j, k=1}^{n} \partial_{y_{j}} \ell_{1}\left(y^{\prime}\right) \partial_{y_{k}} \ell_{1}\left(y^{\prime}\right)\left\{R^{(j)}\left(y^{\prime}, \nabla \widetilde{w}\right) R^{(k)}\left(y^{\prime}, \nabla \widetilde{w}\right)\right. \\
& \left.+s^{2} R^{(j)}(y, \nabla \varphi) R^{(k)}\left(y^{\prime}, \nabla \varphi\right)\right\} \mathrm{d} y \leq \int_{\mathcal{G}_{N}} 2 s G_{\varphi}(y, s, \widetilde{w}) \mathrm{d} y+\int_{\mathcal{G}_{N}}\left\{2 s G_{\phi}(y, s, \widetilde{w})-2 s G_{\varphi}(y, s, \widetilde{w})\right. \\
& \left.+4 N \tau \sum_{j, k=1}^{n} \partial_{y_{j}} \ell_{1}\left(y^{\prime}\right) \partial_{y_{k}} \ell_{1}\left(y^{\prime}\right)\left\{R^{(j)}\left(y^{\prime}, \nabla \widetilde{w}\right) R^{(k)}\left(y^{\prime}, \nabla \widetilde{w}\right)+s^{2} R^{(j)}\left(y^{\prime}, \nabla \varphi\right) R^{(k)}\left(y^{\prime}, \nabla \varphi\right)\right\}\right\} \mathrm{d} y \\
& +C_{8}\left(\frac{1}{s}\left\|L_{2, \phi} \widetilde{w}\right\|_{L^{2}\left(\mathcal{G}_{N}\right)}^{2}+\frac{1}{s}\left\|L_{1, \phi} \widetilde{w}\right\|_{L^{2}\left(\mathcal{G}_{N}\right)}^{2}+s\|\widetilde{w}\|_{L^{2}\left(\partial \mathcal{G}_{N}\right)}\left\|\partial_{y_{n}} \widetilde{w}\right\|_{L^{2}\left(\partial \mathcal{G}_{N}\right)}\right) .
\end{aligned}
$$

Note that there exists a constant $C_{9}>0$, independent of $N$, such that

$$
\int_{\mathcal{G}_{N}} 4 N \tau \sum_{j, k=1}^{n} \partial_{y_{j}} \ell_{1}\left(y^{\prime}\right) \partial_{y_{k}} \ell_{1}\left(y^{\prime}\right)\left\{R^{(j)}\left(y^{\prime}, \nabla \widetilde{w}\right) R^{(k)}\left(y^{\prime}, \nabla \widetilde{w}\right)+s^{2} R^{(j)}\left(y^{\prime}, \nabla \varphi\right) R^{(k)}\left(y^{\prime}, \nabla \varphi\right)\right\} \mathrm{d} y \geq C_{9} N \int_{\mathcal{G}_{N}} \widetilde{w}^{2} \mathrm{~d} y
$$

for all sufficiently large $N$.

By (11), we have

$$
\begin{aligned}
& \int_{\mathcal{G}_{N}}\left(2 s G_{\varphi}(y, s, \widetilde{w})-2 s G_{\phi}(y, s, \widetilde{w})\right. \\
& \left.\quad-4 N \tau \sum_{j, k=1}^{n} \partial_{y_{j}} \ell_{1}\left(y^{\prime}\right) \partial_{y_{k}} \ell_{1}\left(y^{\prime}\right)\left\{R^{(j)}\left(y^{\prime}, \nabla \widetilde{w}\right) R^{(k)}\left(y^{\prime}, \nabla \widetilde{w}\right)+s^{2} R^{(j)}\left(y^{\prime}, \nabla \varphi\right) R^{(k)}\left(y^{\prime}, \nabla \varphi\right)\right\}\right) \mathrm{d} y \\
& \leq C_{10}(N) s \int_{\mathcal{G}_{N}}\left(|\nabla \widetilde{w}|^{2}+s^{2} \widetilde{w}^{2}\right) \mathrm{d} y,
\end{aligned}
$$


where $C_{10}(N) \rightarrow 0$ as $N \rightarrow+\infty$. By $(10)$, we obtain

$$
\begin{aligned}
& \left|\frac{1}{s}\left\|L_{2, \phi} \widetilde{w}\right\|_{L^{2}\left(\mathcal{G}_{N}\right)}^{2}+\frac{1}{s}\left\|L_{1, \phi} \widetilde{w}\right\|_{L^{2}\left(\mathcal{G}_{N}\right)}^{2}-\frac{1}{s}\left\|L_{2, \varphi} \widetilde{w}\right\|_{L^{2}\left(\mathcal{G}_{N}\right)}^{2}-\frac{1}{s}\left\|L_{1, \varphi} \widetilde{w}\right\|_{L^{2}\left(\mathcal{G}_{N}\right)}^{2}\right| \\
& \leq C_{11}(N) s \int_{\mathcal{G}_{N}}\left(|\nabla \widetilde{w}|^{2}+s^{2} \widetilde{w}^{2}\right) \mathrm{d} y,
\end{aligned}
$$

where $C_{11}(N) \rightarrow 0$ as $N \rightarrow+\infty$. Using (25)-(27), from (24) we obtain

$$
\begin{aligned}
\frac{1}{C_{7}} s \int_{\mathcal{G}_{N}}\left(|\nabla \widetilde{w}|^{2}+s^{2} \widetilde{w}^{2}\right) \mathrm{d} y \leq \frac{1}{4}\left\|L_{1, \varphi} \widetilde{w}\right\|_{L^{2}\left(\mathcal{G}_{N}\right)}^{2}+\frac{1}{4}\left\|L_{2, \varphi} \widetilde{w}\right\|_{L^{2}\left(\mathcal{G}_{N}\right)}^{2} & \\
& +\int_{\mathcal{G}_{N}} 2 s G_{\phi}(y, s, \widetilde{w}) \mathrm{d} y+s C_{9}\|\widetilde{w}\|_{L^{2}\left(\partial \mathcal{G}_{N}\right)}\left\|\partial_{y_{n}} \widetilde{w}\right\|_{L^{2}\left(\partial \mathcal{G}_{N}\right)}, \quad \forall s \geq s_{0}(\tau)
\end{aligned}
$$

Inequalities (21), (28) imply (5.28). The proof is finished.

Acknowledgements. Most of this paper has been written during the stays of the first named author at Graduate School of Mathematical Sciences of the University of Tokyo in July and January of 2002 and 2003. The author thanks the school for the hospitality. The authors are deeply indebted to Prof. Kazuhiro Yamamoto for the careful explanation of the results of the paper [49]. The first named author was supported partially by the NSF Grant DMS-0205148. The second named author was supported partly by Grants 15340027 and 15654015 from the Japan Society for the Promotion of Science and the Ministry of Education, Cultures, Sports and Technology.. The authors thank the referees for invaluable comments and suggestions.

\section{REFERENCES}

[1] C. Bardos, G. Lebeau and J. Rauch, Sharp sufficient conditions for the observation, control, and stabilization of waves from the boundary. SIAM J. Control Optim. 30 (1992) 1024-1065.

[2] M. Bellassoued, Distribution of resonances and decay of the local energy for the elastic wave equations. Comm. Math. Phys. 215 (2000) 375-408.

[3] M. Bellassoued, Carleman estimates and decay rate of the local energy for the Neumann problem of elasticity. Progr. Nonlinear Differ. Equations Appl. 46 (2001) 15-36.

[4] M. Bellassoued, Unicité et contrôle pour le système de Lamé. ESAIM: COCV 6 (2001) 561-592.

[5] L. Baudouin and J.-P. Puel, Uniqueness and stability in an inverse problem for the Schrödinger equation. Inverse Problems 18 (2002) 1537-1554.

[6] A.L. Bukhgeim, Introduction to the Theory of Inverse Problems. VSP, Utrecht (2000).

[7] A.L. Bukhgeim, J. Cheng, V. Isakov and M. Yamamoto, Uniqueness in determining damping coefficients in hyperbolic equations, in Analytic Extension Formulas and their Applications, Kluwer, Dordrecht (2001) 27-46.

[8] A.L. Bukhgeim and M.V. Klibanov, Global uniqueness of a class of multidimensional inverse problems. Soviet Math. Dokl. 24 (1981) 244-247.

[9] T. Carleman, Sur un problème d'unicité pour les systèmes d'équations aux derivées partielles à deux variables independantes. Ark. Mat. Astr. Fys. 2B (1939) 1-9.

[10] B. Dehman and L.Robbiano, La propriété du prolongement unique pour un système elliptique. Le système de Lamé. J. Math. Pures Appl. 72 (1993) 475-492.

[11] G. Duvaut and J.L. Lions, Inequalities in Mechanics and Physics. Springer-Verlag, Berlin (1976).

[12] Yu.V. Egorov, Linear Differential Equations of Principal Type. Consultants Bureau New York (1986).

[13] M. Eller, V. Isakov, G. Nakamura and D. Tataru, Uniqueness and stability in the Cauchy problem for Maxwell's and the elasticity system, in Nonlinear Partial Differential Equations, Vol. 14, Collège de France Seminar, Elsevier-Gauthier Villars. Ser. Appl. Math. 31 (2002) 329-350.

[14] M.E. Gurtin, The Linear Theory of Elasticity, in Encyclopedia of Physics, Vol. VIa/2, Mechanics of Solids II, C. Truesdell Ed., Springer-Verlag, Berlin (1972).

[15] L. Hörmander, Linear Partial Differential Operators. Springer-Verlag, Berlin (1963).

[16] M. Ikehata, G. Nakamura and M. Yamamoto, Uniqueness in inverse problems for the isotropic Lamé system. J. Math. Sci. Univ. Tokyo 5 (1998) 627-692. 
[17] O. Imanuvilov, Controllability of parabolic equations. Mat. Sbornik 6 (1995) 109-132.

[18] O. Imanuvilov, On Carleman estimates for hyperbolic equations. Asymptotic Analysis (2002) 32 185-220.

[19] O. Imanuvilov, V. Isakov and M. Yamamoto, An inverse problem for the dynamical Lamé system with two sets of boundary data. Commun. Pure Appl. Math. 56 (2003) 1366-1382.

[20] O. Imanuvilov, V. Isakov and M. Yamamoto, New realization on the pseudoconvexity and its application to an inverse problem (preprint).

[21] O. Imanuvilov and M. Yamamoto, Lipschitz stability in inverse parabolic problems by the Carleman estimate. Inverse Problems 14 (1998) 1229-1245.

[22] O. Imanuvilov and M. Yamamoto, Global Lipschitz stability in an inverse hyperbolic problem by interior observations. Inverse Problems 17 (2001) 717-728.

[23] O. Imanuvilov and M. Yamamoto, Global uniqueness and stability in determining coefficients of wave equations. Commun. Partial Differ. Equations 26 (2001) 1409-1425.

[24] O. Imanuvilov and M. Yamamoto, Determination of a coefficient in an acoustic equation with a single measurement. Inverse Problems 19 (2003) 151-171.

[25] O. Imanuvilov and M. Yamamoto, Remarks on Carleman estimates and controllability for the Lamé system. Journées Équations aux Dérivées Partielles, Forges-les-Eaux, 3-7 juin 2002, GDR 2434 (CNRS) 1-19.

[26] O. Imanuvilov and M. Yamamoto, Carleman inequalities for parabolic equations in Sobolev spaces of negative order and exact controllability for semilinear parabolic equations. Publ. Res. Inst. Math. Sci. 39 (2003) 227-274.

[27] O. Imanuvilov and M. Yamamoto, Carleman estimate for a stationary isotropic Lamé system and the applications. Appl. Anal. 83 (2004) 243-270.

[28] V. Isakov, A nonhyperbolic Cauchy problem for $\square_{b} \square_{c}$ and its applications to elasticity theory. Comm. Pure Appl. Math. 39 (1986) $747-767$.

[29] V. Isakov, Inverse Source Problems. American Mathematical Society, Providence, Rhode Island (1990).

[30] V. Isakov, Inverse Problems for Partial Differential Equations. Springer-Verlag, Berlin (1998).

[31] V. Isakov and M. Yamamoto, Carleman estimate with the Neumann boundary condition and its applications to the observability inequality and inverse hyperbolic problems. Contem. Math. 268 (2000) 191-225.

[32] M.A. Kazemi and M.V. Klibanov, Stability estimates for ill-posed Cauchy problems involving hyperbolic equations and inequalities. Appl. Anal. 50 (1993) 93-102.

[33] A. Khădarov, Carleman estimates and inverse problems for second order hyperbolic equations. Math. USSR Sbornik 58 (1987) 267-277.

[34] A. Khădarov, On stability estimates in multidimensional inverse problems for differential equations. Soviet Math. Dokl. 38 (1989) 614-617.

[35] M.V. Klibanov, Inverse problems and Carleman estimates. Inverse Problems 8 (1992) 575-596.

[36] H. Kumano-go, Pseudo-differential Operators. MIT Press, Cambrige (1981).

[37] I. Lasiecka and R. Triggiani, Control Theory for Partial Differential Equations: Continuous and Approximation Theories. Cambridge University Press, Cambridge (2000).

[38] J.L. Lions, Optimal Control of Systems Governed by Partial Differential Equations. Springer-Verlag, Berlin (1971).

[39] J.L. Lions, Contrôlabilité exacte perturbations et stabilisation de systèmes distribués. Masson, Paris (1988).

[40] J.-P. Puel and M. Yamamoto, On a global estimate in a linear inverse hyperbolic problem. Inverse Problems 12 (1996) 995-1002.

[41] J.-P. Puel and M. Yamamoto, Generic well-posedness in a multidimensional hyperbolic inverse problem. J. Inverse Ill-posed Problems 5 (1997) 55-83.

[42] L. Rachele, An inverse problem in elastodynamics: uniqueness of the wave speeds in the interior. J. Differ. Equations 162 (2000) 300-325.

[43] A. Ruiz, Unique continuation for weak solutions of the wave equation plus a potential. J. Math. Pures. Appl. 71 (1992) $455-467$.

[44] D. Tataru, Carleman estimates and unique continuation for solutions to boundary value problems. J. Math. Pures. Appl. 75 (1996) 367-408.

[45] D. Tataru, A priori estimates of Carleman's type in domains with boundary. J. Math. Pures. Appl. 73 (1994) 355-387.

[46] M. Taylor, Pseudodifferential Operators. Princeton University Press, Princeton, New Jersey (1981).

[47] M. Taylor, Pseudodifferential Operators and Nonlinear PDE. Birkhäuser, Boston (1991).

[48] V.G. Yakhno, Inverse Problems for Differential Equations of Elasticity. Nauka, Novosibirsk (1990).

[49] K. Yamamoto, Singularities of solutions to the boundary value problems for elastic and Maxwell's equations. Japan J. Math. 14 (1988) 119-163.

[50] M. Yamamoto, Uniqueness and stability in multidimensional hyperbolic inverse problems. J. Math. Pures Appl. 78 (1999) $65-98$.

[51] X. Zhang, Explicit observability inequalities for the wave equation with lower order terms by means of Carleman inequalities. SIAM J. Control Optim. 39 (2001) 812-834.

[52] C. Zuily, Uniqueness and Non-uniqueness in the Cauchy Problem. Birkhäuser, Boston, Basel, Berlin, (1983). 